\title{
Signatures of three-nucleon interactions in few-nucleon systems
}

\author{
N. Kalantar-Nayestanaki \\ Kernfysisch Versneller Instituut (KVI), University of Groningen, Zernikelaan 25, \\ 9747 AA Groningen, The Netherlands \\ E-mail: nasser@kvi.nl \\ E. Epelbaum \\ Institute für Theoretische Physik II, Ruhr-Universität Bochum, D-44870 Bochum, \\ Germany \\ E-mail: evgeny.epelbaum@rub.de
}

\section{J.G. Messchendorp}

Kernfysisch Versneller Instituut (KVI), University of Groningen, Zernikelaan 25, 9747 AA Groningen, The Netherlands

E-mail: messchendorp@kvi.nl

\section{A. Nogga}

Institute for Advanced Simulation, Institut für Kernphysik, and Jülich Center for Hadron Physics, Forschungszentrum Jülich, D-52425 Jülich, Germany

E-mail: a.nogga@fz-juelich.de

\begin{abstract}
Recent experimental results in three-body systems have unambiguously shown that calculations based only on nucleon-nucleon forces fail to accurately describe many experimental observables and one needs to include effects which are beyond the realm of the two-body potentials. This conclusion owes its significance to the fact that experiments and calculations can both be performed with a high accuracy. In this review, both theoretical and experimental achievements of the past decade will be underlined. Selected results will be presented. The discussion on the effects of the three-nucleon forces is, however, limited to the hadronic sector. It will be shown that despite the major successes in describing these seemingly simple systems, there are still clear discrepancies between data and the state-of-the-art calculations.
\end{abstract}




\section{Introduction}

The ultimate goal of nuclear physics is to understand the properties of nuclei and reactions involving them. Given the smallness of the typical energy scales in nuclear physics, such as e.g. the nuclear binding energies (BEs), it appears appropriate to formulate the nuclear N-body problem in terms of the non-relativistic Schrödinger equation. In a first approximation, the two-nucleon potential is sufficient to describe the bulk of the few-nucleon observables at low and intermediate energies. At present, a number of semi-phenomenological two-nucleon models are available which provide an accurate description of the nucleon-nucleon (NN) scattering data below the pion production threshold with a $\chi^{2}$ per degree of freedom of the order $\sim 1$. Recent advances in the development of few-body methods coupled with a significant increase in computational resources allow one to perform accurate microscopic calculations of three- and even four-nucleon scattering observables and of the spectra of light nuclei. This opens the door for precise tests of the underlying dynamics and, in particular, of the role and structure of the three-nucleon force $(3 \mathrm{NF})$. One of the simplest and most extensively studied three-nucleon observables is the BE of the triton. It is well known to be significantly underestimated by the existing two-nucleon potentials [1,2] $\$$. A similar underbinding occurs for other light nuclei as well [4]. This is shown in Fig. 1 where the experimental binding energies of light nuclei are compared with exact calculations including two and three-body forces. The need to go beyond the two-nucleon force $(2 \mathrm{NF})$ is rather evident.

Three-nucleon continuum observables have also been explored by several groups. While the differential cross section of elastic nucleon-deuteron $(\mathrm{N} d)$ scattering at incident beam energies below $30 \mathrm{MeV}$ is rather well described using solely NN potentials, a large relative discrepancy with the data, known as the $A_{y}$-puzzle, is observed for the analyzing power; see Fig. 2. The data are shown for energies which are below the breakup threshold. However, the puzzle remains and only diminishes at energies of around $30 \mathrm{MeV}$ [6]. One should, however, note that the actual values of the analyzing powers are rather small so that very small corrections of the nuclear Hamiltonian might be able to resolve this puzzle. We are, therefore, not convinced that this specific observable is a good indicator for significant failures of the $3 \mathrm{~N}$ Hamiltonian. In section 4, we will further discuss this issue also for the $4 \mathrm{~N}$ system. The theoretical results shown in the figure are obtained within the framework of an effective field theory (EFT), see the next section for more details, but are very similar to the predictions of various potential models [7]. Tensor-analyzing powers and spin-transfer coefficients are generally rather well described at low energies using solely $2 \mathrm{NFs}$ but the results of these calculations start to deviate from the data as the energy increases. This was clearly demonstrated for cross sections by Sagara et al. [8, 9]. In addition to the elastic channel, the break-up reaction offers a rich kinematics and as such provides a good testing ground for the

$\ddagger$ Note, however, that phase-equivalent (at low energy) non-local NN potentials can be constructed which reproduce the ${ }^{3} \mathrm{H} B E[3]$. 


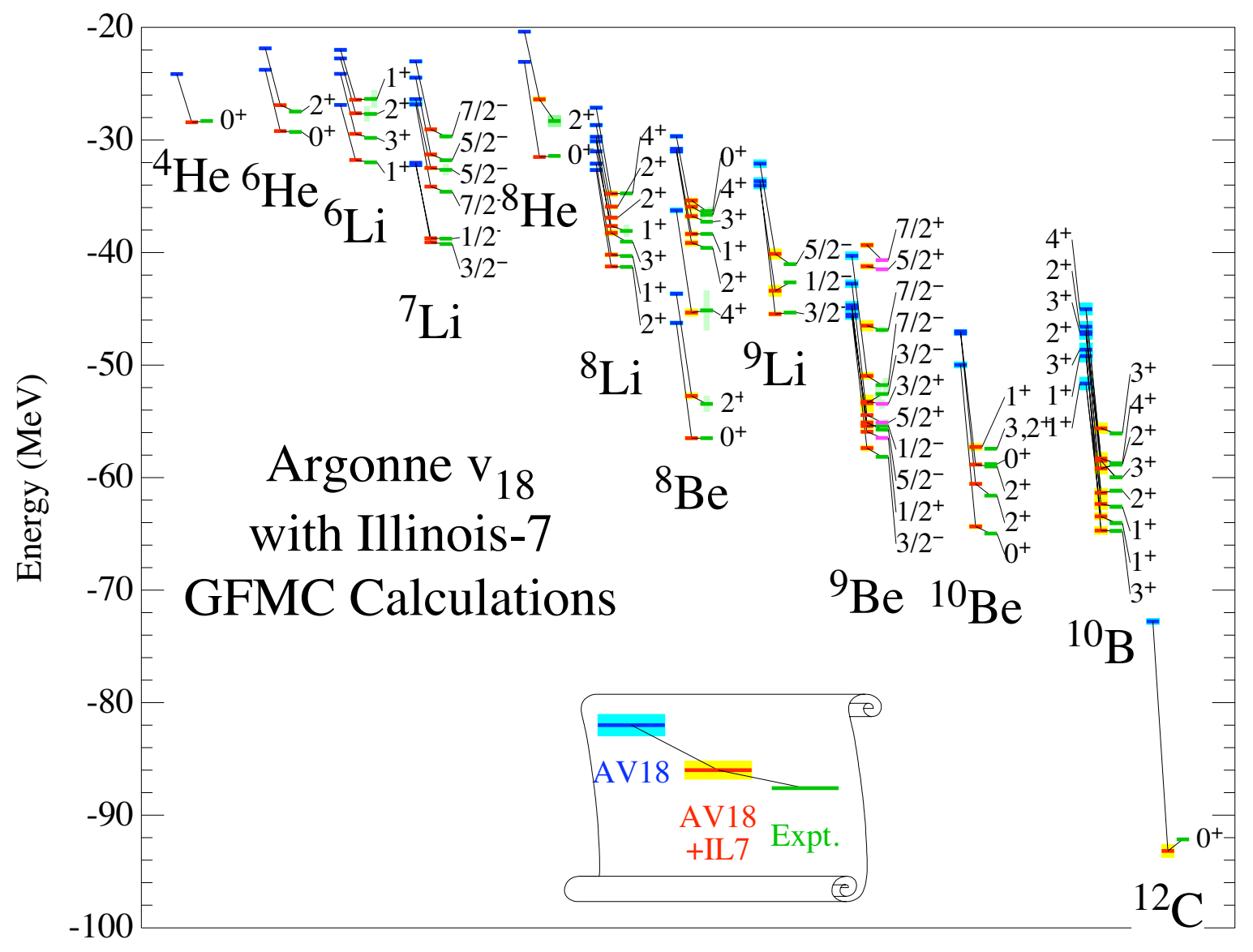

Figure 1. Binding energies of light nuclei. The experimental values are compared with Green's Function Monte Carlo calculations performed with only a two-nucleon potential (AV18, blue/dark grey) and with the addition of a three-nucleon potential (IL7, yellow/light grey) [5] (color online).

structure of the nuclear force. In general, the calculations at low energies agree rather well with the experimental results. However, there are a couple of observables which show major discrepancies with the theoretical predictions. One example is the wellknown space-star kinematics shown in Fig. 3. It should be mentioned, however, that the preliminary results from a recent measurement at Tsukuba tandem laboratory show much a better agreement with the results of the calculations than the data shown in Fig. [3] [10].

The observed discrepancies between data and calculations based solely on 2NFs are usually viewed as an indication of the existence of a 3NF. Indeed, 3NFs which cannot be reduced to pair-wise NN interactions arise naturally in the context of a meson-exchange theory and at the more fundamental level of QCD. At present, several phenomenological 3NF models exist which are typically based on the two-pion exchange contribution and will be discussed in section 3.1. Despite some remarkable successes of the phenomenological approach, many problems still remain open; see section 3.4 for explicit examples. In addition, there are obvious conceptual deficiencies such as e.g. the 


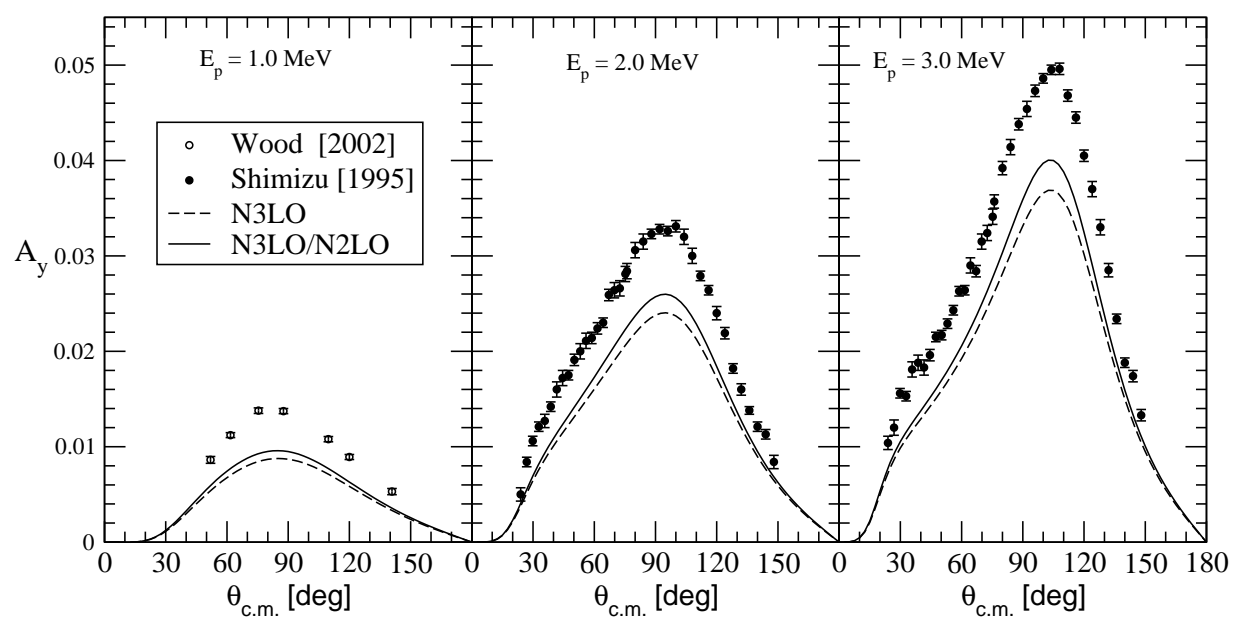

Figure 2. Vector analyzing power in elastic proton-deuteron scattering at three incident proton energies below the break-up threshold. The dashed line represents the result based on the two-nucleon potential derived at next-to-next-to-next-to-leading order chiral EFT while the solid line also includes the effect of the chiral $3 \mathrm{NF}$ at next-to-next-to-leading order [7]. The data are from Refs. [11,12].

lack of a consistent treatment of $2 \mathrm{NFs}$ and $3 \mathrm{NFs}$ in the same framework. On the other hand, significant progress has been achieved recently in understanding the properties of few-nucleon systems within the framework of chiral EFT. This approach is linked to QCD via its symmetries and allows one to analyze the low-energy properties of hadronic systems in a systematic and controlled way. In addition, it offers a natural explanation for the observed hierarchy of nuclear forces: $\left\langle V_{2 N}\right\rangle \gg\left\langle V_{3 N}\right\rangle \gg\left\langle V_{4 N}\right\rangle$.

In this review, some of the experimental observables in proton-deuteron $(p d)$ scattering will be discussed along with the theoretical developments which are taking place. The experimental investigations have been performed at various laboratories for a large part of the phase space. Here, we restrict ourselves mainly to elastic and break-up observables in the medium energy region of 50 to $250 \mathrm{MeV}$ incident nucleon energy.

A number of recent review articles on the nuclear forces and their applications to few- and many-body systems are available. While Refs. [14, 15] focus mainly on applications of EFT techniques to few- and many-nucleon systems, a comprehensive review of the structure of nuclear forces in chiral EFT and selected applications to nucleon-deuteron scattering and light nuclei can be found in Ref. [16]. Recent developments in these and related topics are also considered in a more broadly focused review article [17]. For a broader overview on current topics related to few-nucleon systems, we refer to [18]. Further, a compilation of experimental and theoretical information on the mass 3 systems is presented in Ref. [19]. For a recent review article describing experimental investigations of discrepancies in three-nucleon reactions at low energies see Ref. 9]. Going beyond nuclear systems, for an overview on pionless EFT and universality in few-body systems with large scattering length, the reader is referred 


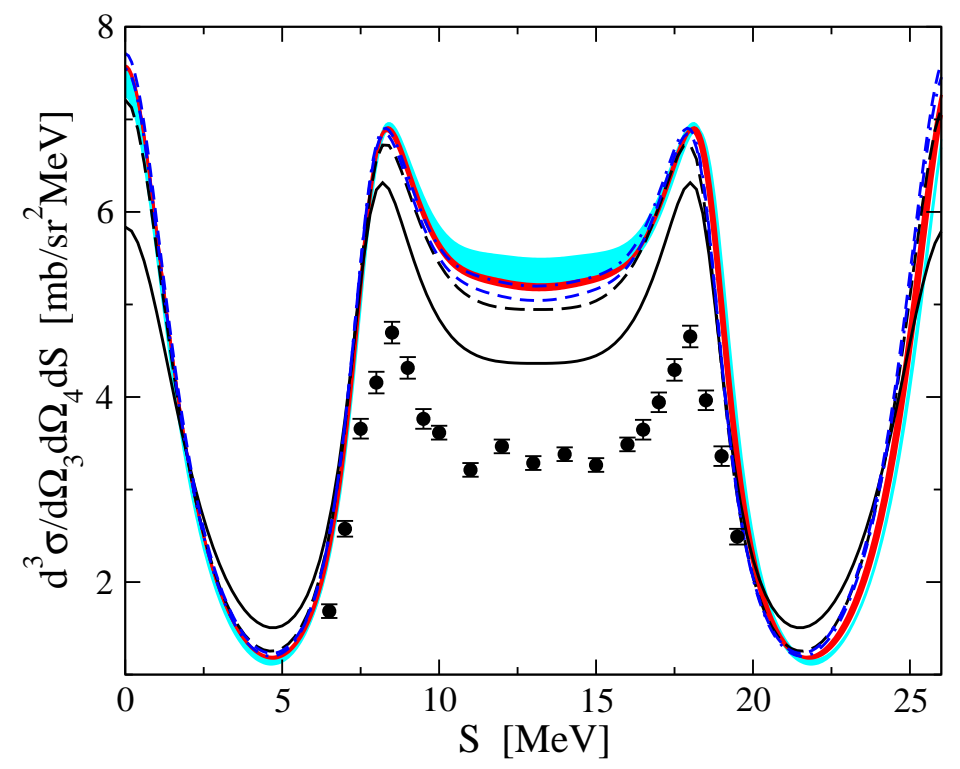

Figure 3. Results for the deuteron break-up cross sections at $E_{d}=19 \mathrm{MeV}$ as a function of the kinematical variable $S$, with $\alpha=56^{\circ}[13$ and taken at the symmetric constant relative energy (SCRE) configuration. Light/cyan (dark/red) shaded bands depict predictions from chiral EFT at NLO $\left(\mathrm{N}^{2} \mathrm{LO}\right)$. Short-dashed and solid (longdashed) lines show the results based on the combination of the CD-Bonn $20002 \mathrm{NF}$ and TM99 3NF and the coupled-channel calculations including the explicit $\Delta$ with (without) Coulomb interaction, respectively. The results based on the CD-Bonn 2000 2NF overlap with the chiral EFT band at $\mathrm{N}^{2} \mathrm{LO}$ (color online).

to [20]. The present review article is focussed more strongly on 3NFs and confronting their effects with experimental data in nucleon-deuteron scattering and, to a lesser extent, four-nucleon scattering and the spectra of light nuclei.

Our paper is organized as follows. In section 2, the nucleon-nucleon force is introduced and briefly discussed. This section is followed by a section on 3NFs and the $A=3$ systems. Latest developments on the theoretical side will be discussed in some detail. In addition, a survey of the experimental data will be made providing the grounds for a systematic comparison with the results of various model calculations. Section 4 is devoted to the discussion of exact calculations of four-body systems and the investigation of the three-body force effects in them. This will be done based on the small existing database for these systems. The manifestation of 3NFs in systems where $A>4$ will be discussed in section 5 . Here, recent theoretical developments will be outlined. Throughout this review, we will limit ourselves to hadronic systems due to lack of space. For a treatment of various aspects of the electromagnetic probe of few-body systems where the role of the $3 \mathrm{NF}$ is also important, we refer the reader to Refs. [21 24]. Notice further that nucleon-nucleon bremsstrahlung and its implications for our knowledge about 3NFs have also been extensively studied [25-46]. Finally, in section 6, conclusions are drawn and an outlook is given for the future. 


\section{Nucleon-nucleon interaction in two-body systems}

\subsection{Theoretical framework}

The nuclear force problem is one of the oldest but still current problems in nuclear physics. It is of a crucial importance for understanding the properties of atomic nuclei and, more generally, strongly interacting hadronic matter. The strong interaction between the nucleons emerges due to the residual color force between quarks and gluons - the elementary constituents of the colorless nucleons. The conventional way to describe the nuclear force utilizes the meson-exchange picture, which goes back to the seminal work by Yukawa [47]. His idea, followed by the experimental discovery of $\pi$ - and heavier mesons $(\rho, \omega, \ldots)$, stimulated the development of boson-exchange models that have laid the foundations for the construction of high-precision phenomenological nucleon-nucleon $(\mathrm{NN})$ potentials. Dispersion relations were also employed to construct the two-pion exchange contribution, see e.g. [48,49].

The most general structure of a non-relativistic two-nucleon potential can be expressed in terms of just a few operators. In momentum space, the operator basis can, for example, be chosen as $\mathbf{1}_{\text {spin }}, \vec{\sigma}_{1} \cdot \vec{\sigma}_{2},\left(\vec{\sigma}_{1} \cdot \vec{q}\right)\left(\vec{\sigma}_{2} \cdot \vec{q}\right),\left(\vec{\sigma}_{1} \cdot \vec{k}\right)\left(\vec{\sigma}_{2} \cdot \vec{k}\right), i\left(\vec{\sigma}_{1}+\vec{\sigma}_{2}\right) \cdot \vec{q} \times \vec{k}$ and $\left(\vec{\sigma}_{1} \cdot \vec{q} \times \vec{k}\right)\left(\vec{\sigma}_{2} \cdot \vec{q} \times \vec{k}\right)$. Here, $\vec{\sigma}_{i}$ are the Pauli spin matrices of the nucleon $i$, $\vec{q} \equiv \vec{p}^{\prime}-\vec{p}, \vec{k} \equiv \vec{p}^{\prime}+\vec{p}$ and $\vec{p}\left(\vec{p}^{\prime}\right)$ refers to initial (final) nucleon momenta in the center-of-mass system. The isospin structure of the two-nucleon force falls into the four different classes [50]: isospin-invariant (class I), isospin-breaking but charge-symmetry conserving (class-II) and charge-symmetry breaking contributions (classes III and IV). Class II interactions break isospin invariance but respect charge symmetry. They are usually referred to as charge-independence breaking and are e.g. responsible for the difference between isovector $n p$ and the average of the $n n$ and $p p$ phase shifts. Class IV (III) forces do (not) cause isospin mixing in the two-nucleon system. For a recent review on charge-symmetry breaking see Ref. [51].

The general strategy in constructing phenomenological potential models such as e.g. the CD-Bonn 2000 [52], Argonne $V_{18}$ (AV18) [53] and Nijmegen I, II potentials [54 involves incorporating the proper long-range tail of the nuclear force due to the electromagnetic interaction (Coulomb interaction [55], vacuum polarization [56, 57] and magnetic moment interaction [58]) and the one-pion exchange potential and parameterizing the medium- and short-range contributions Es. With about 40 to 50 adjustable parameters, these potentials provide an excellent description of a few thousand of low-energy proton-proton and neutron-proton scattering data with $\chi^{2} /$ datum $\sim 1$. We refer the reader to Ref. [59] for a review article on high-precision potentials. For a recent high-precision potential model within the covariant spectator theory see Ref. [60]. Notice that this particular model is designed for using in the manifestly covariant Spectator equations rather than the nonrelativistic Schrödinger

$\S$ In the following, the term phenomenological potential models will be used to refer to the models which reproduce the two-nucleon scattering data with $\chi^{2} /$ datum $\sim 1$, such as the ones specified in the text. 
equations. Effects due to the inclusion of the $\Delta(1232)$ isobar as an explicit degree of freedom have also been investigated. Coupled-channel potential models involving $\mathrm{NN} \rightarrow \mathrm{N} \Delta$ transitions are presented in Refs. [61,62]. In particular, the model of Deltuva et al. 62] based on the CD-Bonn potential leads to a description of the NN data which is comparable to the high-precision phenomenological potentials. It should be noted that the $\Delta \Delta$ channels are not included in this calculation. Note further that the two models of [61, 62] lead to considerably different results for $\mathrm{NN} \rightarrow \mathrm{N} \Delta$ transition amplitudes. These differences are, however, not visible in the two-nucleon observables.

While the conventional approach to the nuclear force problem outlined above enjoys many successes and is frequently used in nuclear structure and reaction calculations, it suffers from having only a very loose connection to QCD. In addition, it does not provide a way for assigning theoretical uncertainties and leaves the question of constructing consistent many-body forces and exchange currents open, see [17] for an extensive discussion.

The obvious drawbacks of the phenomenological framework can be overcome by employing chiral EFT, a systematic and model-independent approach to study the low-energy hadron dynamics in harmony with the symmetry pattern of QCD. This method exploits an approximate, spontaneously broken chiral symmetry of QCD with two flavors of the $u$ - and $d$-quarks and, to a lesser extent, with three flavors of the $u$-, $d$ - and $s$-quarks. These symmetry/symmetry-breaking patterns manifest themselves in the hadron spectrum and provide a natural explanation of the very small (compared to other hadrons) masses of pions which are identified with the corresponding Goldstone bosons. Moreover, the nature of the Goldstone boson implies that pions only interact weakly at low energy. These features are at the heart of chiral perturbation theory. In this framework, low-energy dynamics of pions and nucleons is described in terms of the most general effective Lagrangian formulated in terms of hadronic degrees of freedom and featuring the same chiral symmetry (breaking) pattern as QCD. It contains infinitely many local interactions with increasing number of derivatives and/or quark mass insertions due to the explicit breaking of chiral symmetry. Each term in the effective Lagrangian is multiplied by an a-priori unknown constant, the so-called lowenergy constant (LEC). The values of the LECs are, in principle, calculable from QCD but can also be determined from the data, see e.g. 63. The resulting effective Lagrangian can be applied to compute low-energy pion and single-nucleon observables in a systematic way by making a perturbative expansion in powers of $Q / \Lambda_{\chi}$ [64, 65. Here, $Q$ and $\Lambda_{\chi}$ refer to the soft scale associated with low external momenta or $M_{\pi}$ and the hard chiral-symmetry breaking scale of the order of $1 \mathrm{GeV}$, respectively. A recent review on the methodology and applications of chiral perturbation theory in the Goldstone-Boson and single-nucleon sectors can be found in [66].

Chiral EFT can, however, not be directly applied to low-energy few-nucleon scattering. The strong nature of the nuclear force that manifests itself in the appearance of self-bound atomic nuclei invalidates a naive application of perturbation theory. Weinberg pointed out that the breakdown of perturbation theory can be traced back to 


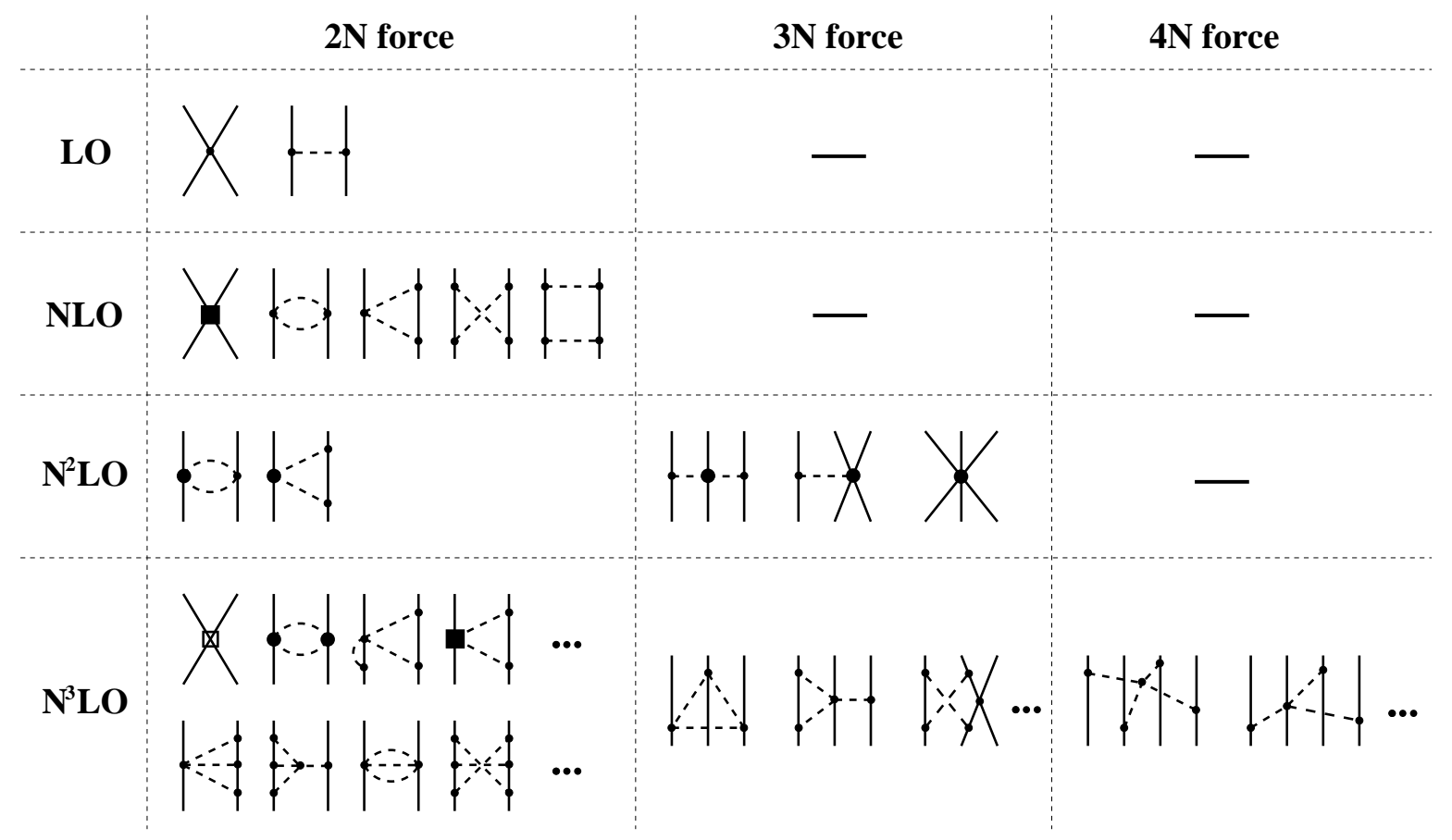

Figure 4. Hierarchy of nuclear forces in chiral EFT based on Weinberg's power counting. Solid and dashed lines denote nucleons and pions, respectively. Solid dots, filled circles and filled squares refer, respectively, to the leading, subleading and subsubleading vertices in the effective Lagrangian. The crossed square denotes $2 \mathrm{~N}$ contact interactions with 4 derivatives.

the infrared enhancement of reducible diagrams that involve few-nucleon cuts [67, 68]. The irreducible part of the amplitude that gives rise to the nuclear force is, however, not affected by the infrared enhancement and is thus accessible within chiral EFT. These important observations have triggered an intense research activity towards the systematic derivation of the nuclear forces in chiral EFT and their applications to the nuclear few- and many-body problem.

It should also be emphasized that an EFT can be formulated that is only valid at typical momenta well below the pion mass. This framework allows to take into account the unnaturally large NN scattering lengths but looses the connection with the chiral symmetry of QCD. It has been used with large success not only for nuclear systems [14,69]. This so-called pion-less EFT requires a 3NF in leading order. The corresponding hard scale is given by the pion mass and, therefore, intermediate-energy observables cannot be predicted within this framework. In the following, we restrict ourselves to chiral EFT.

The EFT expansion of the nuclear force based on the standard chiral power counting (i.e. assuming that all operators in the effective Lagrangian scale according to a naive dimensional analysis) is visualized in Fig. 4. It provides a natural qualitative explanation of the observed hierarchy of two-, three- and more-nucleon forces with 
$\left\langle V_{2 \mathrm{~N}}\right\rangle \gg\left\langle V_{3 \mathrm{~N}}\right\rangle \gg\left\langle V_{4 \mathrm{~N}}\right\rangle \ldots$ The expansion of the two-nucleon force (2NF) has the form

$$
V_{2 \mathrm{~N}}=V_{2 \mathrm{~N}}^{(0)}+V_{2 \mathrm{~N}}^{(2)}+V_{2 \mathrm{~N}}^{(3)}+V_{2 \mathrm{~N}}^{(4)}+\ldots,
$$

with the superscripts referring to the power of the expansion parameter $Q / \Lambda_{\chi}$. The long-range part of the nuclear force is dominated by $1 \pi$-exchange with the $2 \pi$-exchange contributions starting at next-to-leading order (NLO). The expressions for the potential up to next-to-next-to-leading order $\left(\mathrm{N}^{2} \mathrm{LO}\right)$ in the heavy-baryon formulation [70, 71 ] are rather compact and have been independently derived by several authors using a variety of different methods $[7275]$. $2 \pi$ - and $3 \pi$-exchange contributions at next-tonext-to-next-to-leading order $\left(\mathrm{N}^{3} \mathrm{LO}\right)$ have been worked out by Kaiser [76-79] and are considerably more involved, see also Ref. [80]. While the $2 \pi$-exchange at NLO and, especially, at $\mathrm{N}^{2} \mathrm{LO}$ generates a rather strong potential at distances of the order of the inverse pion mass [74], the leading $3 \pi$-exchange contributions turn out to be negligible. The $\mathrm{N}^{3} \mathrm{LO}$ contributions to the $2 \pi$-exchange potential were also derived in the covariant formulation of chiral EFT [81] by Higa et al. [82,83]. The low-energy constants entering the pion-exchange contributions up to $\mathrm{N}^{3} \mathrm{LO}$ are known from pion-nucleon scattering and related processes [84 86]. We note that some of them, especially the ones from $\mathcal{L}_{\pi N}^{(3)}$, are presently not very accurately determined. In the isospin limit, the short-range part of the potential involves 2, 9, 24 independent terms at $\mathrm{LO}, \mathrm{NLO} / \mathrm{N}^{2} \mathrm{LO}, \mathrm{N}^{3} \mathrm{LO}$, respectively. The corresponding LECs were fixed from the two-nucleon data leading to the accurate $\mathrm{N}^{3} \mathrm{LO}$ potentials of Entem and Machleidt (EM) [87] and Epelbaum, Glöckle, Meißner (EGM) [88]. These two potentials differ in the treatment of relativistic corrections (including the form of the employed dynamical equation), isospin-breaking terms and the form of the regulator functions. There are also differences in the adopted values of certain pion-nucleon low-energy constants. Finally, EGM provide an estimation of the theoretical uncertainty by means of the variation of the cutoffs in some natural ranges. This important issue is not addressed in [87], where a single excellent fit to neutronproton (proton-proton) scattering data with $\chi^{2} /$ datum $=1.10\left(\chi^{2} /\right.$ datum $\left.=1.50\right)$ in the energy range from 0 to $290 \mathrm{MeV}$ is given. We refer the reader to Refs. [87, 88] for more details. In Fig. 5, we compare NN phase shifts obtained with these models with predictions from phenomenological potentials from Nijmegen [54], AV18 [53], CDBonn [52] and from Gross and Stadler [60]. We emphasize that the chiral expansion of the two-nucleon scattering observables within the formulations of Refs. [87, 88] is expected to converge at energies well below the pion production threshold. To extend to higher energies, it is necessary to explicitly take into account the momentum scale $Q \sim \sqrt{m_{N} M_{\pi}}$ associated with the real pion production. This has not been pursued so far for two-nucleon scattering. For a detailed discussion of the convergence of the chiral expansion for nucleon-nucleon scattering the reader is referred to Ref. [88].

The consistency of the Weinberg power counting for short-range operators has been questioned by several authors, see e.g. Refs. [91, 92]. The meaning of the non-perturbative renormalization of the Schrödinger equation in the context of chiral EFT and the implications on the power counting are currently under discussion, see 

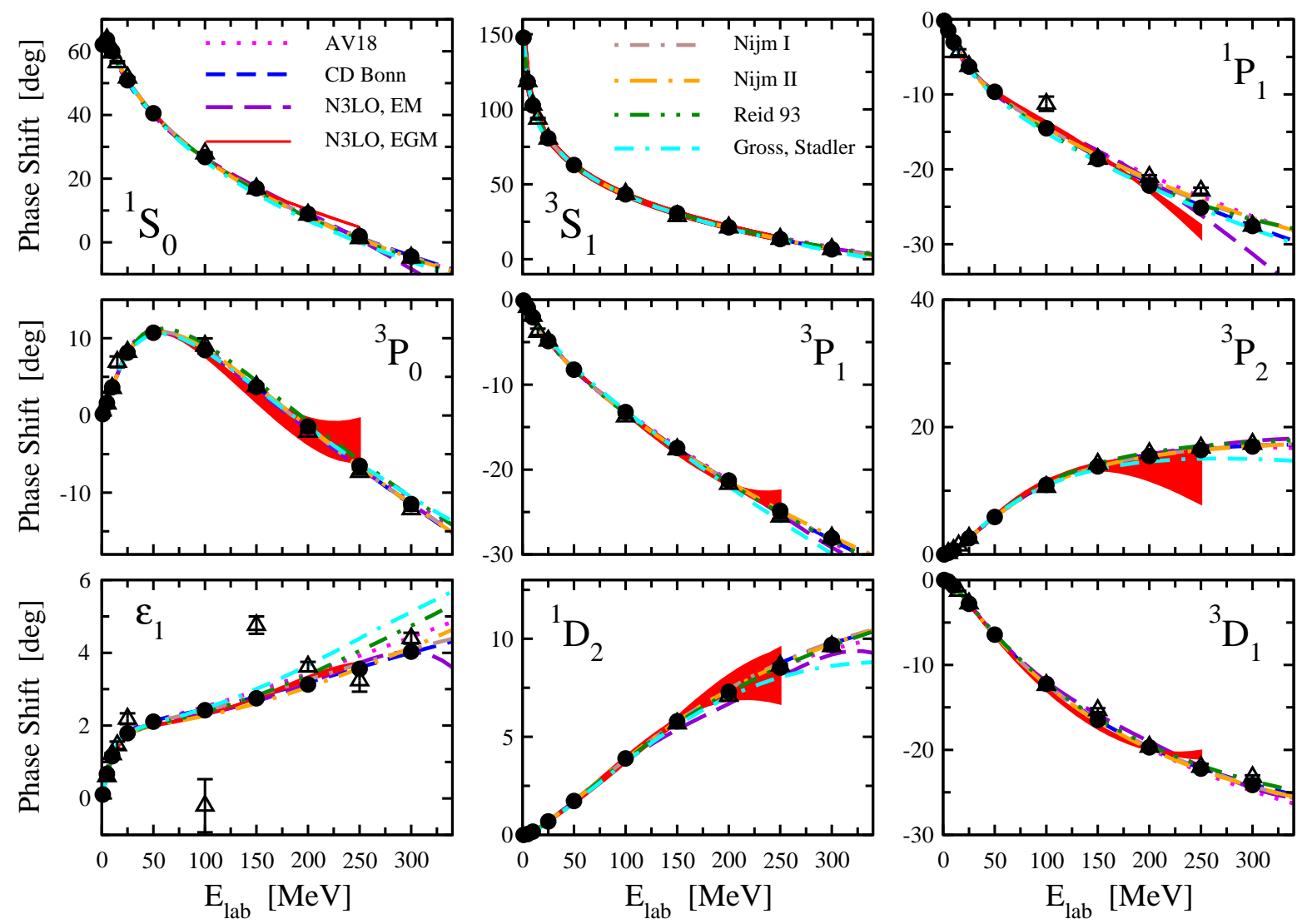

Figure 5. Selected neutron-proton phase shifts for various potentials in comparison with the Nijmegen [89] (filled circles) and Virginia Tech [90] (open triangles) PWA (color online). The band for EGM $\mathrm{N}^{3} \mathrm{LO}$ corresponds to a variation of the cutoffs in the natural range providing a (rough) estimate of the theoretical uncertainty at this order, see Ref. [88] for more details.

Refs. [93 98] and references therein for a sample of different views on this issue. Note that while several different schemes have been proposed in the literature, a real alternative to the Weinberg approach for practical calculations is not yet available.

Isospin-breaking (IB) contributions to the nuclear force have been extensively studied in the framework of chiral EFT. Within the Standard Model, isospin violation has its origin in the different quark masses and the electromagnetic interactions. Chiral EFT is well suited to explore the implications of these two effects which lead to a string of IB terms in the effective hadronic Lagrangian which are proportional either to the quark mass difference or the fine structure constant. IB contributions to the nuclear forces can then be worked out straightforwardly leading to a similar hierarchy for manybody forces as in the isospin-symmetric case. We emphasize, however, that different counting rules are used in the literature to relate the additional expansion parameters for IB contributions (i.e. the quark mass difference and the fine structure constant) with $Q / \Lambda_{\chi}$. In the two-nucleon force, the dominant IB contribution is due to the different pion masses, $M_{\pi^{0}} \neq M_{\pi^{ \pm}}$, in the one-pion exchange. The resulting potential is chargesymmetry conserving, i.e. of class II. Charge-symmetry breaking forces of classes III and 

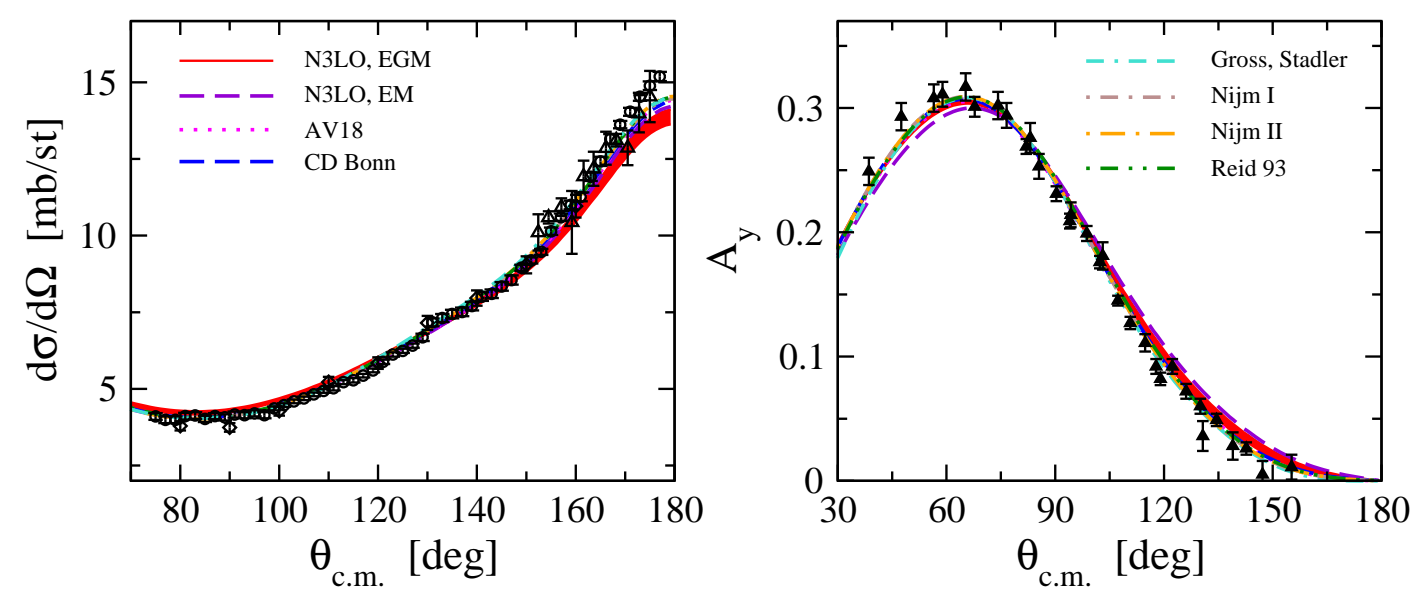

Figure 6. Neutron-proton differential cross section at $E_{\text {lab }}=96 \mathrm{MeV}$ (left panel) and analyzing power at $E_{\text {lab }}=67.5 \mathrm{MeV}$ (right panel) calculated using various potentials. The data for the cross section/analyzing power are from Refs. 108 110] [111] (color online).

IV are considerably weaker and are, to a large extent, driven by the proton-to-neutron mass differences in the one- and two-pion exchange contributions and the short-range terms [99 102]. The power counting suggests the hierarchy of the two-nucleon forces with $\left\langle V_{2 N}^{I}\right\rangle>\left\langle V_{2 N}^{I I}\right\rangle>\left\langle V_{2 N}^{I I I}\right\rangle>\left\langle V_{2 N}^{I V}\right\rangle$ [103] which is consistent with observations. It should also be emphasized that the purely electromagnetic contributions are strongly enhanced under certain kinematical conditions (low energies and/or forward angles) due to their long-range nature. For a more comprehensive review on various contributions to the nuclear force the reader is referred to the recent review articles [14, 16, 17].

All the developments described above are based on the EFT with pions and nucleons as the only degrees of freedom. On the other hand, the $\Delta(1232)$ isobar is known to play an important role in nuclear physics due to its low excitation energy and strong coupling to the $\pi N$ system. The explicit inclusion of the $\Delta$ in the EFT by treating the $\Delta$-N mass splitting as a soft scale [104] allows one to resum a certain class of important contributions leading to an improved convergence. Since the calculations involving the $\Delta$ are considerably more involved, its contribution to the nuclear force are, at present, only worked out up to $\mathrm{N}^{2} \mathrm{LO}$ [72,105-107]. These studies confirm an improved convergence of the EFT expansion compared to the $\Delta$-less theory.

\subsection{Observables and comparison with experimental data}

Both the phenomenological potentials and the ones resulting from chiral EFT at $\mathrm{N}^{3} \mathrm{LO}$ allow for an accurate description of the low-energy nucleon-nucleon scattering data and the deuteron properties. As representative examples, we show in Fig. 6 the neutronproton differential cross section at $E_{\text {lab }}=96 \mathrm{MeV}$ and vector analyzing power at $E_{\text {lab }}=67.5 \mathrm{MeV}$, for which fairly recent data are available. 
It is worth saying a few words about the fitting procedure. In most cases, the unknown parameters contributing to the isospin $T=1$ channels are determined solely from the more accurate proton-proton scattering data. The neutron-proton potentials in the $T=1$ channels are then reconstructed from the corresponding proton-proton ones by employing corrections for the Coulomb interaction and certain isospin-breaking effects while the $T=0$ part is fixed to the neutron-proton data. The precise form of isospin-breaking corrections varies between the different models. Note that the same procedure is also adopted in the Nijmegen 1993 partial wave analysis (PWA). In this context we would like to emphasize, that isospin-breaking effects in the two-nucleon force lead to sizable effects in the neutron-deuteron $A_{y}$, see e.g. [112]. Clearly, the neutronneutron potentials also have to be reconstructed from the corresponding proton-proton and neutron-proton ones since no neutron-neutron scattering data are available. The phenomenological potentials are typically fitted to the data below $350 \mathrm{MeV}$, while the EM N ${ }^{3} \mathrm{LO}$ potential uses the data below $290 \mathrm{MeV}$. The LECs entering the EGM N ${ }^{3} \mathrm{LO}$ potential are determined from a fit to the Nijmegen PWA at energies below $100 \ldots 200$ $\mathrm{MeV}$ (depending on the partial wave).

As already pointed out in the previous section, the available phenomenological nucleon-nucleon potentials provide excellent fits of the scattering data below the pion production threshold with $\chi^{2}$ datum $\sim 1$ or slightly above. We further emphasize that one usually allows for some rescaling of the data in order to minimize the resulting $\chi^{2}$. Notice further that different groups adopt somewhat different criteria to reject inconsistent data. For example, the data set used in the recent model of Gross and Stadler [60] includes 3788 neutron-proton data, 3336/3010 of which are prior to 2000/1993 while rejecting 1180 data points which are found to be statistically inconsistent. To compare, the Nijmegen PWA was fit to 2514, AV18 to 2526 and CDBonn 2000 to $3058 \mathrm{np}$ data. A set of 53 neutron-proton data for the differential cross section at $96 \mathrm{MeV}$ and $75^{\circ} \leq \theta_{\mathrm{CM}} \leq 179^{\circ}$ from Ref. [110] shown in the left panel of Fig. 6] is an example of data that have been rejected from the PWA. It yields $\chi^{2} /$ datum $\approx 2.8$ for the current solution of the Virginia Tech PWA [90]. We also emphasize that a very precise recent measurement of neutron-proton $A_{y}$ at $12 \mathrm{MeV}$ at TUNL [113] raised some debate about a possible $A_{y}$ problem in low-energy neutron-proton scattering, see Ref. [114] and references therein. The last issue is possibly related to the $A_{y}$ problem in low-energy $\mathrm{N} d$ scattering mentioned earlier [112]. For our later discussions on $3 \mathrm{NF}$ effects at intermediate energies, the differences in the fitting procedures, the strategies for rejecting data and minor inconsistencies of different PWAs are not significant. Last but not least, we would also like to emphasize the large difference between the Nijmegen and Virginia Tech PWA regarding the values of the mixing angle $\epsilon_{1}$, see Fig. 5, indicating that the available neutron-proton scattering data are not very sensitive to this particular observable. 


\section{Three-nucleon forces and $A=3$ systems}

\subsection{NF models}

As discussed in the introduction, it is by now clear that one needs additional interactions beyond pair forces to describe nuclei and nuclear reactions accurately. Such interactions are many-body forces and it is believed that the most important many-body interactions are 3NFs. In this section, we briefly summarize the current status of 3NF models with a special emphasis on models which were used to study few-nucleon systems. For a more complete overview, the reader is referred to [115].

Qualitatively, the importance of many-body forces for nuclei has already been realized in the early days of nuclear physics [116]. In the 1950s, the pion field theory was extensively used to derive nuclear forces. In this era, there were attempts to derive 3NFs on the same footing as NN interactions [117]. However, it turned out to be impossible to obtain a quantitative description of nuclear interactions in this framework due to the lack of a systematic expansion parameter, so that the community finally turned to a combination of theoretical insights and phenomenology to develop nuclear interactions culminating in today's phenomenological and accurate NN interaction models. With this development, NN and 3N forces were not derived anymore on the same grounds. Instead it was tried to make use of dispersion relations to link the interactions to $\pi \mathrm{N}$ scattering. The most prominent early $3 \mathrm{NF}$ obtained in this way is the famous Fujita-Miyazawa force [118] that is still at the heart of phenomenological 3NFs. It was assumed that the most important contribution to the $3 \mathrm{~N}$ interaction is given by the left-most topology in Fig. 7. The blob encodes the $\pi \mathrm{N}$ scattering amplitude. For the $3 \mathrm{NF}$, this amplitude is required below threshold so that dispersion integrals were needed to determine the $3 \mathrm{NF}$ quantitatively. In this way, it is found that by far the most important contribution emerges from $p$-waves and can be linked to $\Delta$ isobars in intermediate states.

The development of today's most widely used 3NF models began in the 1970s and early 1980s based on the work by Fujita and Miyazawa [118] or the later work using current algebra by Brown and Green [119] or McKellar and Rajaraman [120]. The Tucson-Melbourne collaboration studied the latter approach in more detail [121, 122] using current algebra relations to constrain the $\pi \mathrm{N}$ scattering amplitude. This lead to the widely used Tucson-Melbourne (TM) 3NF. Originally, the interaction contained one more term than the Fujita-Miyazawa force (see the discussion below).

Around the same time, the Urbana group found that for a decent description of saturation of nuclear matter, a short ranged repulsive interaction is required. They simplified the $2 \pi$ exchange ansatz of [118] to include only the $\Delta$ part but added a purely phenomenological short-range term. The force was then adjusted to reproduce the triton binding energy and the nuclear matter saturation density in conjunction with a specific NN interaction [123]. This lead to a series of 3NFs called Urbana. The most up-to-date version adjusted in conjunction with AV18 is called Urbana-IX [124].

In parallel, Robilotta and others developed a $3 \mathrm{NF}$ model based on a $\pi \mathrm{N}$ amplitude

from chiral Lagrangians resulting in $3 N_{F}$ very similar to the one of the Tucson- 
Signatures of three-nucleon interactions in few-nucleon systems

$\mathrm{N}^{2} \mathrm{LO}:$
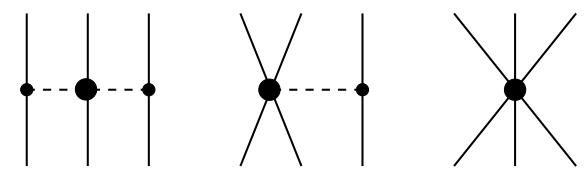

$\mathrm{N}^{3} \mathrm{LO}:$
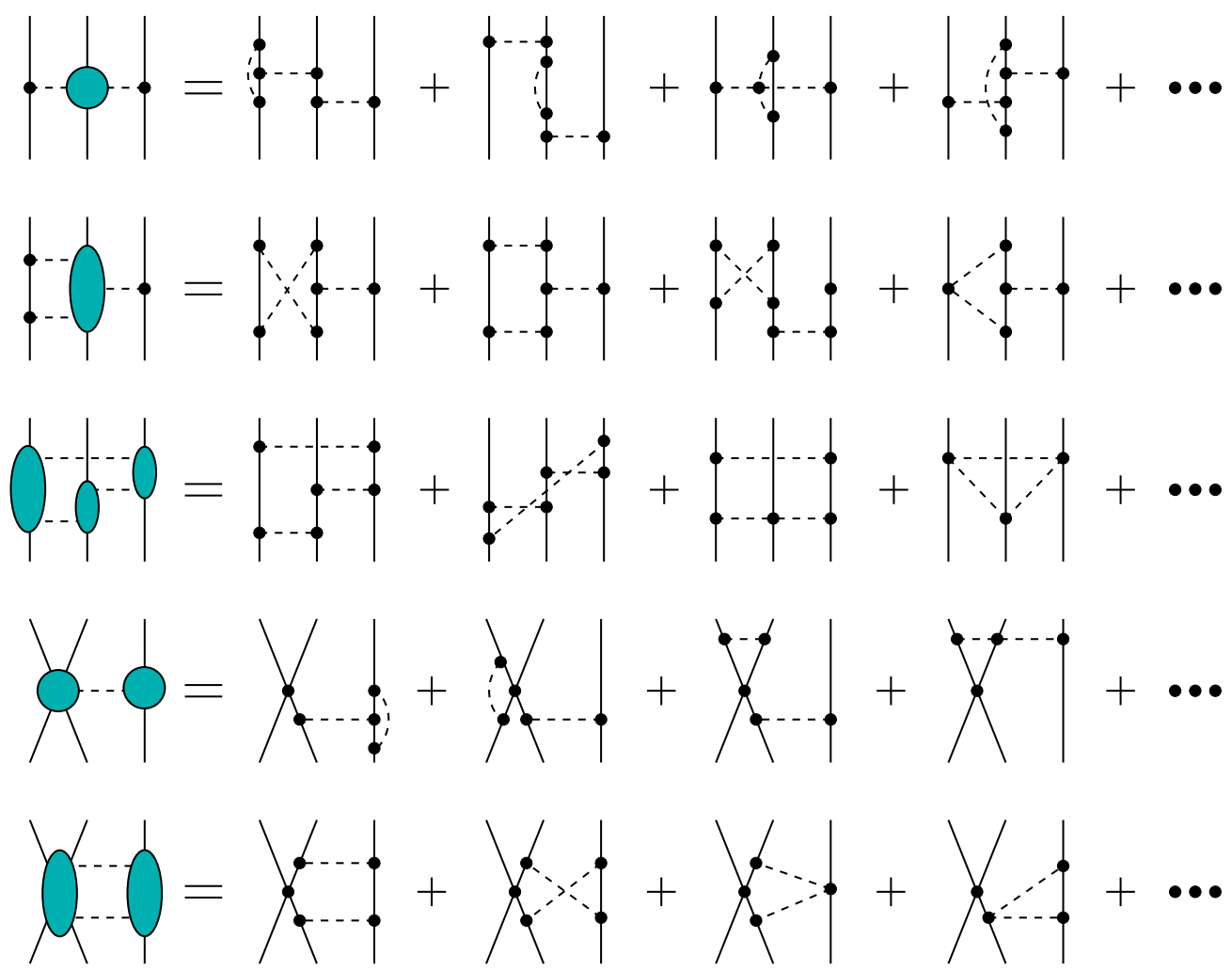

Figure 7. Different topologies contributing to the leading and subleading $3 \mathrm{NF}$ in chiral EFT.

Melbourne group [125] although a specific term of the orginal TM force was missing. Contrary to their expectations, they found that the $2 \pi$-exchange part of the interaction depends strongly on the cutoff parameter employed in the models [125, 126] which resulted in a very strong dependence of, e.g. the triton binding energy on the chosen cutoff parameter. Their analysis showed that this feature is related to contact interaction terms which were part of the $2 \pi$-exchange ansatz, and they argued that these parts should be removed [126] which resulted in a further difference to TM.

In Ref. [127], this issue was re-examined in the framework of chiral EFT. In this framework, there are terms beyond the $2 \pi$ exchange (see below). But it turns out that the $2 \pi$ exchange contribution has a similar structure as the $3 \mathrm{NF}$ of Ref. [125]. Specifically, it misses the additional term in the TM interaction. Ref. [127] recommends to remove the additional term from the TM force. This finally lead to a new version [128] of the Tucson-Melbourne force commonly called TM'. We will present predictions of this model later on in this review. Note that TM' (as the chiral 3NF) keeps the short range 
part of the $2 \pi$-exchange and that, for TM', the dependence on the cutoff is used to adjust the 3NF in conjunction with different NN models to the triton binding energy [129].

In the framework of EFT, the cutoff dependence is removed by the additional topologies of Fig. 77, which enable one to absorb the dependence on the short range part of the $3 \mathrm{NF}$ in additional contact terms.

As a further improvement of the TM model, shorter-ranged contributions were considered in Refs. [130, 131]. Their effect on the ${ }^{3} \mathrm{H}$ binding energy was studied in [132 134] showing that they reduce the binding energy. Unfortunately, their effect on intermediate energy $n d$ scattering has not been systematically studied yet. Recently, however, there is new interest in such interactions [135] and their effect on intermediate energy observables will be studied in the near future.

The most important contribution to the $3 \mathrm{NFs}$ discussed so far can be linked to intermediate excitations of the nucleons to $\Delta$ isobars. This motivated the HanoverLisbon group to study few-nucleon systems in a coupled-channel framework that allows for explicit $\Delta$ excitations (see section 22). Then, few-nucleon calculations automatically contain terms related to intermediate $\Delta$ excitations. These terms are, however, not completely equivalent to corresponding $3 \mathrm{NFs}$ since the $\Delta \mathrm{s}$ are not treated in a static approximation and also mesons other than pions are taken into account. The shortrange part generated by the heavy-meson exchanges is probably, to some extent, taken into account by the adjustment of cutoff parameters of the 3NFs discussed previously. But at least the dispersive corrections due to non-static $\Delta$ s do not have a counter part in standard 3NFs models, and they are known to be non-negligible for the triton binding energy [136] and $\mathrm{N} d$ scattering [137]. The coupled-channel approach has the obvious advantage that all parameters are fixed by two-body observables and the properties of few-nucleon systems are predicted. On the other hand, there is no systematic way to incorporate other effects into the 3NFs. Therefore, once the model fails to reproduce some data, systematic improvements are difficult. This is already the case for the triton binding energy which is slightly underpredicted in this approach.

The calculations carried out by the Hanover-Lisbon group also include the Coulomb interaction making a direct comparison to $p d$ scattering data possible [138, 139]. Recent studies showed that the Coulomb interaction becomes important specifically in elastic forward scattering and in break-up configurations where the two protons have a small relative momentum [140 142. Full calculations based on a purely nucleonic NN potential and standard 3NF models only recently became available [142 144]. The effect of the Coulomb interaction using the AV18 NN potential and the Urbana IX $3 \mathrm{NF}$ was shown 142 to give similar results compared to that of the calculations using the intermediate $\Delta$ approach. For a large part of the available experimental data, the Coulomb effect is sizeable with respect to the 3NF effects. From this perspective, it is favourable to use the calculations by the Hanover-Lisbon group as a benchmark for the global analysis of the world database. On top of this, 3NF effects can be isolated using the interaction model with or without an explicit $\Delta$ resonance [62. Hence, a systematic comparison between data and predictions by the Hanover-Lisbon group provides us 
with a way to systematically study the role of $3 \mathrm{NFs}$ without the ambiguity of the role of the Coulomb effect. It turns out that in many cases the $3 \mathrm{NF}$ effect due to explicit $\Delta \mathrm{s}$ is very similar to the effects of the standard 3NF models. To quantify this statement, a comparison has been made for proton-deuteron elastic-scattering cross sections calculated for an energy range between 50 to $250 \mathrm{MeV}$. This comparison is presented in Fig. 8 where the difference is shown between the results of the calculations by Bochum-Cracow group using the CD-Bonn NN force and the TM' 3NF and those by the Hanover-Lisbon group using the CD-Bonn NN potential but now with the explicit inclusion of the $\Delta$ (both without taking the Coulomb effects into account). In the left panel, the relative difference in cross-section predictions of both approaches using only the two-nucleon CD-Bonn potential are shown where one observes very small differences as one would naively expect. In the right panel, the relative differences are again plotted including $3 \mathrm{NF}$ effects in both models. One can see that, depending on the scattering angle, differences of up to $15 \%$ emerge between the two model calculations. The above mentioned model dependence should, therefore, be taken into account when making definitive conclusions. On the other hand, the standard calculations can be performed for many different combinations of $\mathrm{NN}$ and $3 \mathrm{~N}$ force. This will allow to quantify the model dependence and to pin down observables that are sensitive to the spin-isospin structure of 3NFs. Therefore, the combination of both theoretical approaches will be required to analyze the existing data with high precision.

As mentioned above, with the invention of phenomenological potential models of the $\mathrm{NN}$ interaction, the development of 3NFs became separated from the NN interactions. While one part of the community was discussing the identification of observables that allow one to determine the "off-shell" part of NN interactions to develop a "proper" $\mathrm{NN}$ force [27 30] the other part was studying the properties of the different 3NF models realizing that the short-range part of the $2 \pi$-exchange interactions is strongly dependent on details of the $3 \mathrm{NF}$, like cutoffs [126]. It then became clear that off-shell effects are not measurable [145] and the NN and 3N interactions cannot be studied independently of each other [146, 147]. Different choices for the NN force can be made equivalent by adding proper 3NFs. Conversley, there is no unique 3NF as (parts of the) $3 \mathrm{NF}$ can be traded for off-shell NN interactions, see Refs. [60, 148] for an explicit example. Within the model approach, there were attempts to derive $\mathrm{NN}$ and $3 \mathrm{~N}$ interactions on the same grounds [149] but, with the invent of phenomenological potentials, it became a common practice to adjust the $3 \mathrm{~N}$ interaction models (using e.g. a cutoff parameter) in conjunction with one of the phenomenological NN models to reproduce at least the ${ }^{3} \mathrm{H}$ binding energy (like it always has been done for the Urbana series of models) [129]. This approach had a phenomenological success. It turns out that many low-energy observables scale with the triton binding energy in the sense that all models adjusted in this way will predict the observables equally well|l .

\|It turns out that such correlations are a universal consequence of the large nucleon-nucleon scattering length and also show up in atomic and molecular systems. This can be understood based on pion-less EFT [20, 69, 150, 151]. 


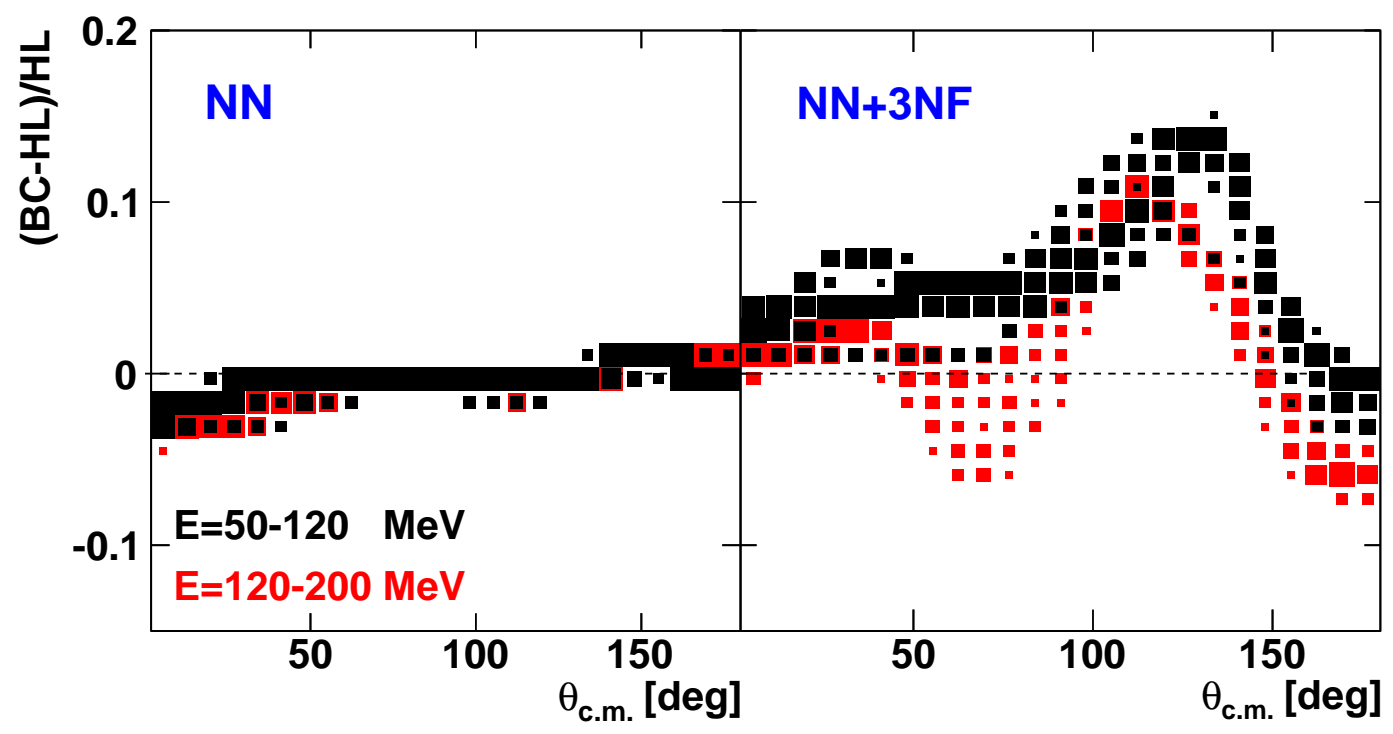

Figure 8. The relative difference between the predictions of the Bochum-Cracow (BC) and Hanover-Lisbon (HL) groups for proton-deuteron elastic-scattering cross sections as a function of $\theta_{\text {c.m. }}$. for a number of incident nucleon energies between 50 to $200 \mathrm{MeV}$. In the left panel, the differences between the models are shown for the case when only the two-nucleon CD-Bonn potential has been used. For the right panel, the threenucleon force effects have also been included in both calculations (TM' for BC and the $\Delta$ in the case of HL). The results are grouped in two bands, one for $50-120 \mathrm{MeV}$ with black squares and the other for 120-200 MeV with grey/red squares (color online). In all calculations, Coulomb effects have been discarded.

This adjustment of the strength of the $3 \mathrm{NF}$ in conjuction with $2 \mathrm{~N}$ force has been a major step towards a quantitative description of few-nucleon systems. The adjusted 3NFs and the different NN interactions gave a series of nuclear Hamiltonians that describe the low energy $3 \mathrm{~N}$ scattering observables very well. The few exceptions became known as puzzles (see the discussion of $A_{y}$ and the space star anomaly below). However, the description of the binding energies of $p$-shell nuclei and of intermediate-energy scattering observables is worse and still dependent on the chosen model clearly showing that the $3 \mathrm{~N}$ Hamiltonian is not fully understood. Based on the models, it became possible to identify observables that are probably most useful to study the structure, spin and isospin dependence of $3 \mathrm{~N}$ forces [152] which triggered a series of experiments that increased the database of three-nucleon scattering observables tremendously (see discussion in section 3.3). We will discuss the data in comparison to model calculations below.

Obviously, there is also a major interest to understand the binding and excitation energies of light nuclei based on NN and 3N interactions. Since these are very sensitive to small contributions to the Hamiltonian, the calculations also showed failures of the models [4,153. For the AV18 and Urbana-IX potentials, the failures were traced back to the isospin dependence of the force. Therefore, the Argonne-Los Alamos-Urbana collaboration added a new structure motivated by $3 \pi$-exchanges with intermediate $\Delta$ 
isobars to their model culminating in the series of Illinois interactions [154]. For light nuclei, the model improved the description of the data, but e.g. going to neutron matter, today's realizations lead to predictions that are not in line with masses and radii of observed neutron stars [155].

Besides all the previously described developments on 3NFs, relativistic corrections to the generally used non-relativistic framework might also contribute to the discrepancy between the theory and data. In the $3 \mathrm{~N}$ systems, relativistic effects have thoroughly been studied for the bound state [156,157], elastic [158] and break-up [159] nd scattering. The effects on the triton binding energy are somewhat dependent on the scheme with which the non-relativisitic NN interaction was matched to the relativistic one. Generally, the effect is repulsive such that even larger $3 \mathrm{NF}$ effects can be expected. Also the dependence on the NN model is not reduced. Therefore, clearly, 3NFs will be required to resolve the underbinding problem for nuclear bound states. For scattering, relativistic effects were usually found to be small even at intermediate energies. Only for some specific breakup configurations, the effects were sizable. We note that $A_{y}$ at low energy is somewhat affected by relativistic effects [160]. A complete calculation however showed that they are far too small to resolve the well-known puzzle and even worsen the agreement with the data [161]. Based on these results, we conclude that the inclusion of the relativistic corrections along these lines does not allow to remove discrepancies between predictions and data. On the other hand, the calculations by Gross and Stadler carried out within the covariant spectator theory [60,148] demonstrate that the correct triton binding energy can be obtained from a relativistic kernel consisting only from oneboson exchange terms, provided one makes a specific choice for the off-shell behavior of the boson-nucleon couplings. The results of these studies provide yet another explicit evidence of the non-uniqueness of the nuclear Hamiltonian and its separation into twoand three-nucleon operators. It is, however, not clear to what extent the findings of [60,148] emerge due to the relativistic treatment of the nuclear dynamics.

In summary, we have today a set of nuclear interaction models based on NN and $3 \mathrm{~N}$ forces that describe nuclear systems fairly well, but still show failures. It is conceivable that these failures are due to missing structures in the $3 \mathrm{~N}$ forces. Improvement of the models is much more difficult than in the case of $\mathrm{NN}$ interactions since the number of possible momentum, spin and isospin structures is much higher. Whereas it was possible to use the most general operator structure of the NN interaction allowed by the symmetries as the starting point for the models, this is not feasible for the $3 \mathrm{~N}$ interactions. We, therefore, need more insight to pin down the most important structure of $3 \mathrm{~N}$ interactions. The models discussed so far are not systematically improvable and are, therefore, limited in providing detailed insight in 3NFs.

The most promising approach to improve nuclear interactions is presently chiral EFT as discussed in section 2. At this point, mostly the version without explicit $\Delta$ degrees of freedom has been used to formulate consistent $\mathrm{NN}$ and $3 \mathrm{~N}$ (and even many-body) interactions. In the formulation based on the pions and nucleons as the only explicit degrees of freedom, the first non-vanishing $3 \mathrm{NF}$ emerges at $\mathrm{N}^{2} \mathrm{LO}$ in the 
chiral expansion (as long as one uses energy-independent formulations) and consists of only three different topologies [162, 163], see Fig. 7. One of them is identical to the $2 \pi$ exchange which is the base of all phenomenological $3 \mathrm{NF}$ models. Its strength, however, is related to the strength of the corresponding terms in the NN force [16]. This underlines the need of consistency in $\mathrm{NN}$ and $3 \mathrm{~N}$ interactions. Two additional topologies are of a shorter range. The strength of the $1 \pi$-exchange contribution (Fig. 7 , middle diagram) is related to various other processes such as e.g. pion production in two-nucleon collisions [164] or ${ }^{3} \mathrm{H}$ beta decay [165]. The weak decay has already been used in [166] to constrain the 3NF. In the available few-nucleon studies, the two lowenergy constants related to the shorter-range topologies (middle and right diagrams in Fig. 77) are usually determined from few-nucleon observables. The ${ }^{3} \mathrm{H}$ binding energy is generally used as one constraint on these parameters. As a second constraint, often the ${ }^{4} \mathrm{He}$ radius or binding energy or the $n d$ doublet scattering length are used. Although these observables are correlated with the ${ }^{3} \mathrm{H}$ binding energy, it turns out that an accurate description with phenomenological interactions is only possible once the short-ranged 3NF terms are included in the calculations (see e.g. [167]).

From the two-nucleon system, we know already that $\mathrm{N}^{2} \mathrm{LO}$ only allows for a quantitative description of low-energy data. Only at $\mathrm{N}^{3} \mathrm{LO}$, the two-nucleon system is described equally well by chiral interactions as by the phenomenological potential models up to intermediate energies. This carries over to three-nucleon observables. The predictions for these observables become rather strongly dependent on the cutoff at $\mathrm{N}^{2} \mathrm{LO}$ making them useful for a quantitative comparison with the data only at energies below $100 \mathrm{MeV}$. Therefore, it is of utmost importance to extend now also the 3NF to $\mathrm{N}^{3} \mathrm{LO}$. In part, this has been achieved in Refs. [168, 169] for the long-range part of the interaction. The remaining parts have also been worked out and are to be

published [170]. At this order, there are several new structures, and it will be interesting to study their impact on few-nucleon observables in future. Here, the power of chiral EFT becomes apparent. Not only that the power counting helped to identify the more important structures of the $3 \mathrm{NF}$, but also the strength of the individual terms is related to other parts of the Hamiltonian. This implies that the number of parameters of the $3 \mathrm{NF}$ that need to be fit to few-nucleon data is the same in order $\mathrm{N}^{2} \mathrm{LO}$ and $\mathrm{N}^{3} \mathrm{LO}$. For a recent work on the short-range terms beyond $\mathrm{N}^{3} \mathrm{LO}$ see Ref. [171].

\subsection{Experimental techniques for measuring the observables}

In the past decades, detection systems suitable to study certain aspects of the dynamics of the three-body systems have been developed at various laboratories. The exact form and characteristics of a detection system clearly depend on the reaction and the observable one is studying. For example, in the study of the cross section of elastic $p d$ scattering, one needs an unpolarized source of protons or deuterons and a small detector which is capable of identifying one of the outgoing particles with a high energy/angle resolution. For this reaction, the best detector would then be a magnetic spectrometer 
with a small solid angle. The measurement of the analyzing power of the reaction would require the use of polarized sources of particles. To measure more complicated observables such as spin-correlation coefficients one would need a polarized target as well, and the investigation of spin-transfer coefficients would require, instead, the measurement of the polarization of the outgoing particles. Each one of these reactions will have its complications although most techniques are now well under control. In this section, some experimental aspects relevant for this field will be discussed.

The use of polarized beams has been very common in the past two decades. There are three types of polarized-ion sources: optically-pumped source, Lamb-shift source, and the atomic-beam source [172 177]. At the facilities where few-nucleon systems are studied at intermediate energies, most sources are based on the latter type. In all these sources, polarizations of $60-80 \%$ of the maximum theoretical value have been achieved. For a review of these sources, see [178]. The Lamb-shift principle has also been used in the measurement of the degree of polarization of protons and deuterons [179 181].

The (polarized) beams of protons and deuterons are accelerated by different techniques depending on the energy required. The low-energy measurements (below $25 \mathrm{MeV}$ ) were generally performed using a (Tandem) Van de Graaff accelerator. At higher energies, one uses cyclotrons to accelerate particles up to a few hundred $\mathrm{MeV}$. At IUCF, Uppsala and Jülich, particles are also accelerated in rings [182]. Since the beam has gone through a secondary stage of acceleration to achieve higher energies, one should perform polarimetry of the beam in order to determine the degree of the polarization of the beam. This is done using a polarimeter which is based on measuring the azimuthal asymmetries of scattered particles and knowing the analyzing powers of a well-known reaction such as proton-proton, proton-deuteron, and proton-Carbon elastic scattering. At lower energies, elastic $p-{ }^{3} \mathrm{He}$ as well as ${ }^{3} \mathrm{He}(d, p){ }^{4} \mathrm{He}$ have also been used. For a description of the principle of operation of these polarimeters, the reader is referred to [183, 184] for intermediate energy polarimeters (between 100 to $200 \mathrm{MeV}$ outgoing particle energies) and [185] for the low-energy ones (below $30 \mathrm{MeV}$ ).

In addition to protons and deuterons, neutron beams have also extensively been used at TUNL, Bonn, Erlangen, PSI, Uppsala, LANSCE and RCNP [108,186 195]. The obvious disadvantage of the neutron beam is that it cannot be manipulated in the beam lines so that experiments are more difficult. The beam intensities are also generally lower than proton or deuteron beams since neutrons are produced as a secondary beam of particles. In contrast to charged-particle detectors where efficiencies close to $100 \%$ can be reached, the efficiency of the neutron detectors is generally lower and requires difficult calibration [196]. All this is not particularly a problem in these reactions as most of them involve only hadronic vertices producing high counting rates and the challenges have been tackled since Coulomb distortions are absent when one uses neutron beams.

The (polarized) beams of particles impinge on different targets of interest. For the three-body studies, proton and deuteron beams are combined with deuteron and proton targets, respectively. In the past, one used a thin foil of solid $\mathrm{CH}_{2}$ or $\mathrm{CD}_{2}$. These targets are easy to manufacture with a high accuracy in the target thickness. The main 
disadvantage of these targets is, however, the background originating from the carbon content of the target. Measurements had to be done on pure carbon targets to measure independently the contribution of, in general, unwanted background. With the advent of very thin (down to a few $\mu \mathrm{m}$ ) and strong synthetic foils, liquid targets became more popular and in particular at intermediate energies where the straggling through target posed less of a problem [197,198. These targets have the advantage of less background from the target window but their thickness has to be determined every time the target is warmed up as the bulging of the foils, and therefore the target thickness, varies by a few percent. Finally, for the ring experiments, one has to use very thin targets in the form of gas or microparticles [199,200]. The very low target density is then compensated by the fact that the beam revolution frequency in the ring is of the order of $1 \mathrm{MHz}$ increasing the effective luminosity by a factor of $10^{6}$.

As mentioned above, the design of the detection system depends on the reaction, the configuration of interest and the energy of the particles to be detected. Magnetic spectrometers with small solid angles but a high energy and angle resolution have been used in the study of the elastic scattering processes. As an example, a spectrum is shown in Fig. 9 for proton-deuteron scattering from a solid $\mathrm{CD}_{2}$ target. Despite the fact that a coincidence measurement has reduced the background drastically, the picture shows clearly the need to have a separate measurement on carbon to understand and subtract the background to obtain high accuracies. Most results discussed in this review article for the elastic-scattering channel have been obtained with the use of spectrometers for the detection of protons and deuterons.

To measure more complicated spin observables such as spin-transfer coefficients or spin-correlation coefficients, one needs to measure the polarization of the outgoing particle in the former case or have a polarized target in combination with a polarized incident beam for the latter case. Successful attempts have been made in the past to measure both observables. At low energies, this has been done at Cologne and Bonn with the help of a polarimeter [185, 201]. At intermediate energies, several polarimeters have been designed and operated [202 206]. Since these measurements require a secondary scattering, both the statistical and systematic uncertainties will be larger than those of analyzing powers for instance. Due to kinematical constraints (low energy of the outgoing particles), the angular ranges of the measurements are also generally more limited than in the case of other observables. The measurements of spincorrelations coefficients have been done only at IUCF with the help of the polarized target used in the ring [182]. An overview of the IUCF-PINTEX setup can be found in Ref. [207] describing extensively the polarized beams and targets for all their double spin experiments. Polarized gas targets have also been developed elsewhere for various purposes (see Refs. [208,209] for a general discussion on this). Solid polarized targets from Ammonia have also been produced successfully in the past [210] but have never been used in the realm of few-body physics.

In the nucleon-deuteron break-up studies, there will be three particles in the final state and, hence, the detection system becomes more complicated. In this case, one 

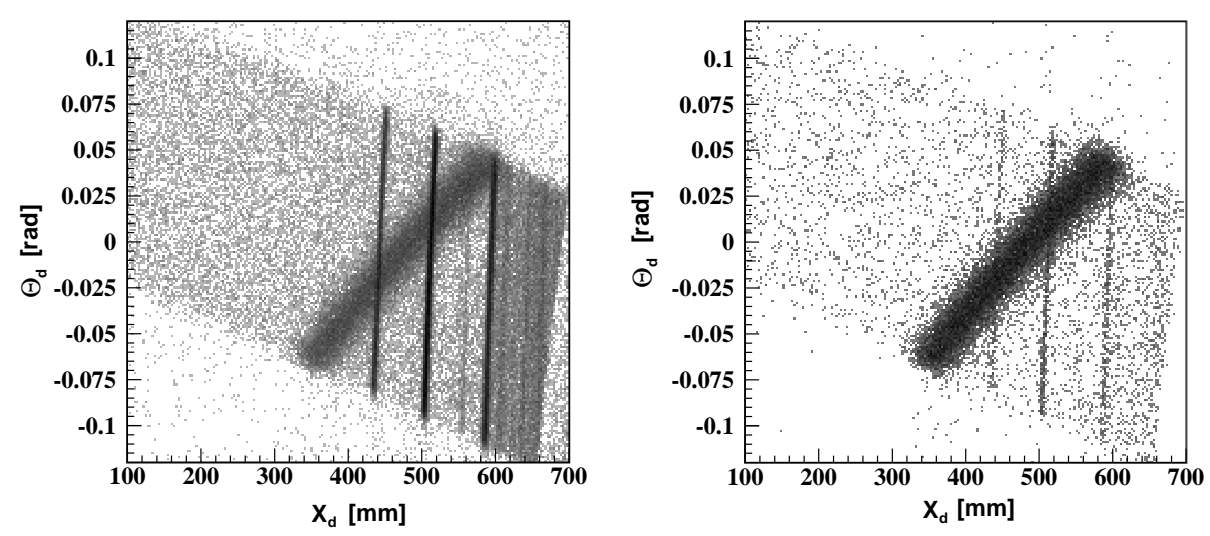

Figure 9. A spectrum of deuterons emerging at a laboratory angle of $41^{\circ}$ from protondeuteron scattering on a solid $\mathrm{CD}_{2}$ target with a proton beam of $150 \mathrm{MeV}$. One can clearly see the peak of interest on a smooth background and a few excited states from the carbon content. The reason for the skewedness of the deuteron peak is the light mass of the particle entering the magnetic spectrometer (kinematical broadening). On the left, the spectrum is measured with deuterons entering the magnetic spectrometer and on the right, a coincidence has been made with a small scintillator detector placed at the appropiate angle for elastic proton-deuteron scattering.

could either choose a very specific geometry in the measurement or employ a detector with a very large acceptance to accommodate for the detection of more than one particle in a large part of the reaction phase space. All the low-energy measurements performed at Cologne [211], Bonn [212], Durham (TUNL) [186 188, and Kyushu [8] and also the first studies at an energy of $65 \mathrm{MeV} /$ nucleon [213 216] have opted for the first choice and looked at the break-up reaction for selected kinematics such as FSI, QSF, SST, SCRE etc.. Based on the experience gained from these measurements and earlier work at KVI [217, 218], a $4 \pi$ detection system was designed and built recently. This detection system, which is in operation since 2005, is shown in Fig. 10 and is capable of detecting light ions (protons, deuterons etc.), and partly neutrons, down to very low energies (a few MeVs in the backward part of the detector). It also acts as a polarimeter for the beam as it has a $\phi$ coverage of $360^{\circ}$. The break-up data discussed in this review have been obtained mostly using detectors similar to the one discussed in Refs. [217,219] or shown in Fig. 10, Figure 11] shows a typical measurement performed in the break-up experiments in which the energy of the first outgoing particle (proton) is plotted against that of the second one (proton). Due to energy and momentum conservation, the detection of two particles in the final state already overdetermines the kinematics, thereby giving an extra handle to reduce the already low background of these measurements. The cross sections are generally studied as a function of the arc-length $S$ for a given bin in $S$ as shown by the two lines in Fig. 11 and for a fixed angular combination of the two outgoing protons. Alternative ways of presenting the data also exist in the literature [220]. 


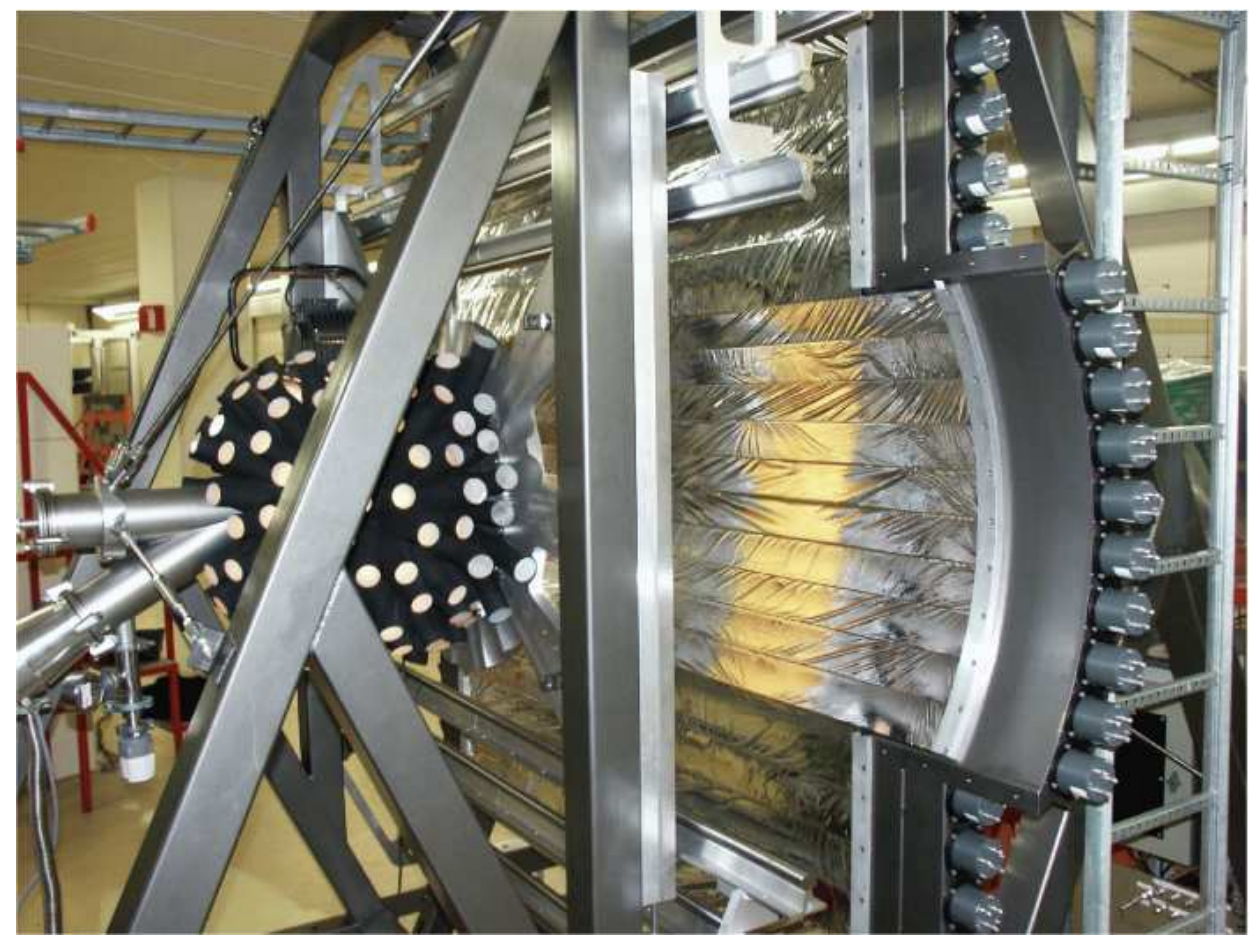

Figure 10. The $4 \pi$ detection system, BINA (Big Instrument for Nuclear-polarization Analysis) at KVI. For presentation purposes, the phototubes in the backward ball and all the cables have been removed. Also the wire chamber placed to measure the particles moving to forward angles of less than $45^{\circ}$ is not shown (color online).

\subsection{Survey of the experimental database for $A=3$ systems}

In the study of three-nucleon force effects, many laboratories have produced data in the past three decades. Most of the data at low energies (up to $30 \mathrm{MeV}$ ), have been produced at TUNL with (polarized) neutrons, protons and deuterons [186 188], Cologne [13, 221 229], Bonn [201], Madison [230], and Kyushu with protons and deuterons [8]. Measurements have also taken place with the injector of the cyclotron at RCNP at a deuteron energy of $26 \mathrm{MeV}$. The observables include cross sections, analyzing powers and spin-transfer coefficients. The focus of this paper is on the results at intermediate energies, from $50 \mathrm{MeV} /$ nucleon to energies around the pion-production threshold. Various observables in elastic and break-up reactions, such as differential cross sections, analyzing powers, spin correlations, and spin-transfer coefficients, have been measured at these energies exploiting (polarized) beams of protons and deuterons, partly in combination with polarized targets. The beams of (polarized) protons and deuterons have been delivered by cyclotrons, such as those at KVI (65-190 MeV/nucleon) [218, 231 244], RIKEN (70-140 MeV/nucleon) [245-248], RCNP (250 MeV/nucleon) [195], PSI (formerly known as SIN) (65 MeV/nucleon) [213 216, 249, 250, Harvard (146 MeV/nucleon) [251], and IUCF (70-200 MeV/nucleon) [252], synchrocyclotrons such as those at Berkeley (145-220 MeV/nucleon [253], Orsay (155 MeV/nucleon) [254], and Rochester (198 MeV/nucleon) 255], synchrotrons such as that at SATURNE (95- 


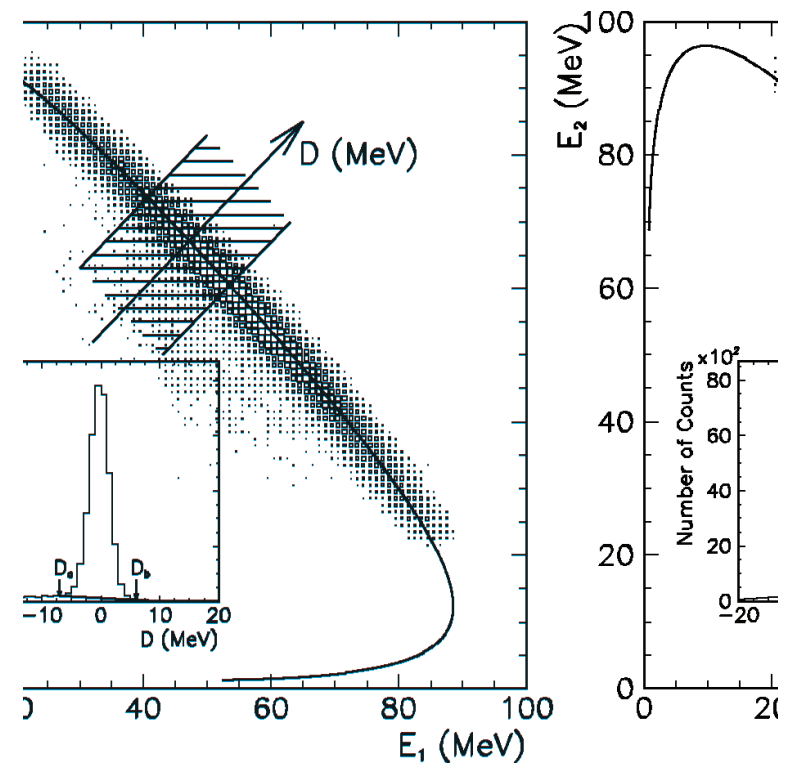

Figure 11. The measured energy correlation between the two outgoing protons in the break-up reaction from proton-deuteron scattering for a certain combination of proton angles at a beam energy of $65 \mathrm{MeV} /$ nucleon. The kinematical locus, the socalled $S$-curve is drawn for this combination and the data follow the expected pattern with little background. A typical $S$-bin is indicated together with the way the data are projected on the axis perpendicular to this axis, D-axis. In the inset, the projected data are shown with little background. Reprinted with permission from [218]. Copyright (2003) by the American Physical Society.

$200 \mathrm{MeV} /$ nucleon for intermediate energies) [256], or accelerated in a ring such as the one at IUCF (135 and $200 \mathrm{MeV} /$ nucleon) [257,258. Experiments at much higher energies of up to $1.3 \mathrm{GeV}$ were also performed at SATURNE. In addition, neutron beams have been used at PSI (up to $65 \mathrm{MeV}$ ) [193], Uppsala (at $95 \mathrm{MeV}$ ) [259] and at LANSCe $(140-240 \mathrm{MeV})$ [260 to study the three-body system with the advantage of having no Coulomb effect which, otherwise has to be accounted for in the calculations. The obvious disadvantage of the neutron beams is the quality and the intensity of the beams. Elastic scattering, the break-up reaction and radiative capture have been studied with these beams.

An overview of what has been measured up until now can be seen in Fig. 12, This figure is inspired by a graph presented by K. Sekiguchi at various conferences. As can be seen from the figure, the density of the points is reduced as one attempts to measure more complicated observables. For instance, measurements at only a few energies have been performed for the spin-correlation coefficients which requires a polarized beam and a polarized target. This has been achieved at the IUCF ring with very thin polarized gas-jet targets. Note, however, that the spin-correlation coefficients, denoted by $C_{i j}$ in Fig. 12, for the elastic $\mathrm{N} d$ channel correspond to 10 observables measured over a wide angular range. The density of the points is also higher for energies below the pionproduction threshold. This has to do with the fact that the opening of this inelasticity 
Nd elastic scattering

\begin{tabular}{|c|c|c|c|c|}
\hline & \multicolumn{4}{|c|}{200} \\
\hline$\frac{\mathrm{d} \sigma}{\mathrm{d} \Omega}$ & 0 & & & 0 \\
\hline $\overrightarrow{\mathrm{n}} \overrightarrow{\mathrm{n}} \mathrm{A}_{\mathrm{y}}(\mathrm{N})$ & 0 & C) & a & $\varphi$ \\
\hline $\overrightarrow{\mathrm{d}} \mathrm{A}_{\mathrm{y}}(\mathrm{d})$ & $0 !$ & 0 & 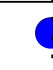 & \\
\hline $\mathrm{A}_{\mathrm{yy}}$ & • & 0 & 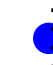 & : \\
\hline$A_{x x}$ & 0 & 0 & 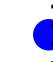 & \\
\hline $\mathrm{A}_{\mathrm{XZ}}$ & 0 & 0 & 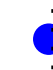 & $\vdots$ \\
\hline $\overrightarrow{\mathrm{p}} \rightarrow \overrightarrow{\mathrm{p}}$ & & & & : \\
\hline $\mathrm{K}_{\mathrm{i}}^{\mathrm{j}^{\prime}}$ & & & & $\bullet$ \\
\hline $\overrightarrow{\mathrm{d}} \rightarrow \overrightarrow{\mathrm{p}}$ & & & & \\
\hline $\mathrm{K}_{\mathrm{y}}^{\mathrm{y}}$ & •: & & & \\
\hline $\mathrm{K}_{\mathrm{ij}}^{\mathrm{y}}$ & & & & \\
\hline $\overrightarrow{\mathrm{p}}+\overrightarrow{\mathrm{d}}$ & & & & \\
\hline $\mathrm{C}_{\mathrm{ij}}$ & & 0 & 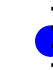 & \\
\hline
\end{tabular}

Nd break-up

\begin{tabular}{|c|ccccc|}
\hline & \multicolumn{3}{|c}{100} & \multicolumn{2}{c|}{200} \\
\hline $\mathrm{d} \sigma$ & $\bullet$ & $\bullet$ & $\bullet$ & $\vdots$ \\
\hline $\mathrm{d} \Omega$ & & & $\bullet$ & $\vdots$ & $\vdots$ \\
\hline $\overrightarrow{\mathrm{p}} \mathrm{A}_{\mathrm{y}}$ & $\bullet$ & & $\bullet$ & $\bullet$ & $\vdots$ \\
$\mathrm{A}_{\mathrm{Z}}$ & & $\bullet$ & $\bullet$ & $\vdots$ & $\vdots$ \\
\hline $\overrightarrow{\mathrm{d}} \mathrm{A}_{\mathrm{y}}(\mathrm{d})$ & $\bullet$ & $\bullet$ & $\bullet$ & $\vdots$ & $\vdots$ \\
$\mathrm{A}_{\mathrm{yy}}$ & $\bullet$ & $\bullet$ & $\bullet$ & $\vdots$ & $\vdots$ \\
$\mathrm{A}_{\mathrm{Xx}}$ & $\bullet$ & $\vdots$ & $\bullet$ & $\vdots$ & $\vdots$ \\
$\mathrm{A}_{\mathrm{Xz}}$ & & $\vdots$ & $\bullet$ & $\vdots$ & $\vdots$ \\
\hline $\overrightarrow{\mathrm{p}} \rightarrow \overrightarrow{\mathrm{p}}$ & & $\vdots$ & $\vdots$ & $\vdots$ & $\vdots$ \\
$\mathrm{K}_{\mathrm{i}}^{\mathrm{j}}$ & & $\vdots$ & $\vdots$ & $\vdots$ & $\vdots$ \\
\hline $\overrightarrow{\mathrm{d}} \rightarrow \overrightarrow{\mathrm{p}}$ & & $\vdots$ & $\vdots$ & $\vdots$ & $\vdots$ \\
$\mathrm{K}_{\mathrm{yy}}^{\mathrm{y}}$ & & $\vdots$ & $\bullet$ & $\vdots$ & $\vdots$ \\
& & $\vdots$ & $\vdots$ & $\vdots$ & $\vdots$ \\
\hline $\overrightarrow{\mathrm{p}}+\overrightarrow{\mathrm{d}}$ & & $\vdots$ & $\vdots$ & $\vdots$ & $\vdots$ \\
$\mathrm{C}_{\mathrm{ij}}$ & & $\vdots$ & $\bullet$ & $\vdots$ & $\vdots$ \\
\hline
\end{tabular}

Figure 12. Overview of the observables measured at various laboratories with beams of neutrons (grey/red squares), protons and deuterons (grey/blue circles) with different energies (in units of $\mathrm{MeV}$ per nucleon). The size of each circle or square roughly represents the angular coverage for a particular observable at a given energy. A large circle or square refers to a (nearly) complete angular coverage, whereas for a small circle or square only a limited angular range was measured. Open circles refer to data that are presently being analyzed and not yet published (color online).

complicates the calculations to the extent to which it becomes difficult to draw any conclusions about the nature of the three-nucleon forces. Also the number of points measured with the neutron beams is clearly less than that measured with proton or deuteron beams due to the difficulty in producing high-quality neutron beams.

\subsection{A comparison of experimental data with various models}

The wealth of the data in the three-body systems can be presented in this short review neither in a tabular form nor in a graphical form. To familiarize the reader with the observables, several observables are first shown as a function of the kinematical variable as they were originally published but for a couple of energies. Out of all measured observables presented in this paper, some of them clearly demonstrate that ab-initio Faddeev calculations based on phenomenological NN potentials, even after including the Coulomb force, are not capable of describing the data in the case only two-nucleon 


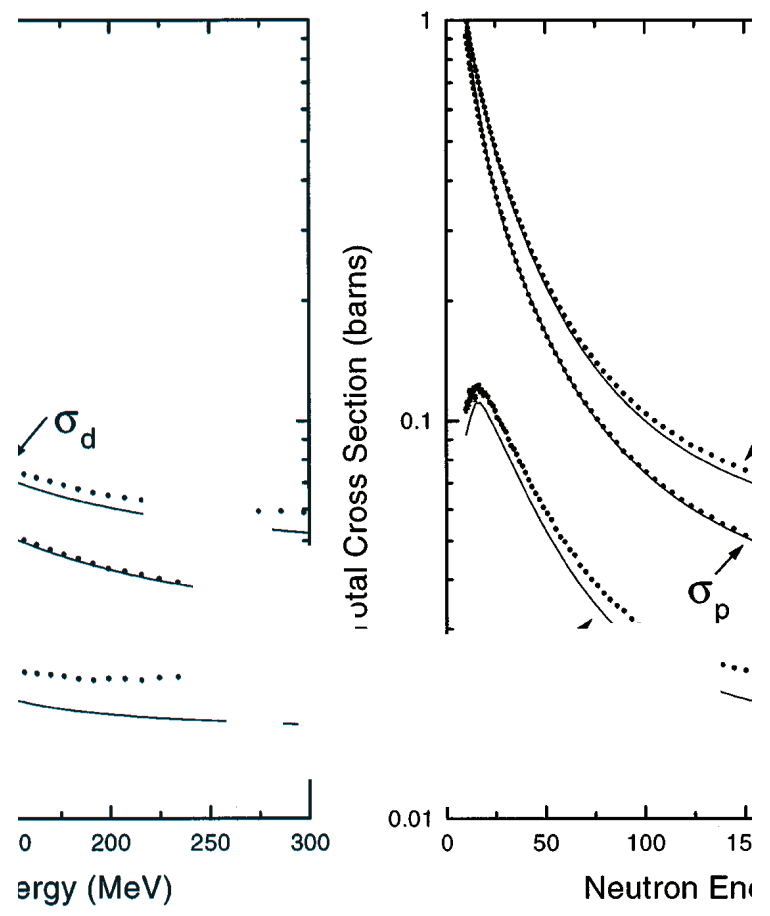

Figure 13. Total neutron-proton and neutron-deuteron cross sections as a function of incident neutron energy [261]. The solid curves are the results of the calculations for the two- and three-body systems exploiting only $2 \mathrm{NF}$. The dotted lines are data. Reprinted with permission from 261. Copyright (1998) by the American Physical Society.

forces are included. In addition, it will be shown that for the same observables, the addition of a phenomenological three-body forces is giving contradicting results. These conclusions will then been globally presented in figures which we refer to as a "global analysis". An attempt has been made to select all the available data and compare them with the calculations on a very global scale. In this way, the details of individual experiments and possible problems with them will be obscured. However, (new) features can be observed which would then point to places where more attention has to be paid to.

As illustrated in section 2.1, nuclear forces obey a certain hierarchy implying that $3 \mathrm{NF}$ effects are much smaller, on the average, than $2 \mathrm{NFs}$. This can be very nicely demonstrated by the inclusive total $n p$ and $n d$ scattering data measured at Los Alamos [261] as shown in Fig. 13. First calculations including the 3NF [262] have shown that current models of the $3 \mathrm{NF}$ can explain approximately $1 / 2$ of deviation of the calculations from the data. Since a recent study of relativistic effects [263] indicates that these cannot resolve the problem, it can be expected that an improved $3 \mathrm{NF}$ will remove the remaining discrepancies. Whether shorter range 3NFs can help to resolve this issue needs to be seen in future. These observations demonstrate the need for exclusive measurements which can provide a significantly larger sensitivity to 3NF effects for specific regions in phase space or for other observables than total cross sections. 
The differential observables which are chosen for the discussions are selected at a lower and a higher energy in the energy range relevant for the discussion of this paper (between 50-250 MeV/nucleon). They are shown in Figs. 14 and 15 for the low and high energies, respectively, and are differential cross sections and proton analyzing powers (top row), deuteron vector and tensor analyzing powers (second row), selected spincorrelation coefficients (third row) and selected spin-transfer coefficients (fourth row) for elastic scattering.

In Fig. 16, one can see five-fold differential cross sections, vector and tensor analyzing powers for two specific configurations at a low energy of $65 \mathrm{MeV} /$ nucleon for the three-body break-up reaction. Other configurations but now for a high-energy beam of protons of $190 \mathrm{MeV}$ are presented in Fig. 17. The number of data points for spin-transfer coefficients and spin-correlation coefficients are very small [258, 264] and therefore not presented.

From Figs. 14, 15, 16, and 17 the following observations can be made. The statistical precision of the data set is almost everywhere in the phase space very high except at higher energies where the cross sections are generally small, and for the spintransfer coefficients for which a secondary scattering is necessary and the spin-correlation coefficients which requires polarized beam and target. The systematic uncertainties are, on the other hand, generally the dominating sources of errors. For cross sections, these come from the determination of the target thickness, the beam current, and the acceptances and the efficiencies of the detection systems and are generally in the order of $5 \%$ of the value of the cross sections. For the analyzing powers, a large fraction of uncertainties cancel out or remain as a second-order correction when ratios of cross sections are calculated. In this case, the main source for uncertainties comes from the precision at which the beam (target) polarization can be measured, which is usually around $2-4 \%$ of the value of the measured spin observables.

The second observation is that for almost all observables in elastic scattering, the calculations which only include 2NFs fail to a large extent to describe the data, and in particular at higher energies. The effect is very large in the minimum of the elasticscattering cross section as was first pointed out by Witała et al. [266] and Nemoto et al. 267. It was exactly in this region, where the first searches for the effects of $3 \mathrm{NF}$ were conducted. It is also very intuitive that this should be the case, since it is in the minimum of the cross section where the $2 \mathrm{NF}$ effects become very small allowing other small effects to be relatively enhanced. However, it can also be noted that even adding the $3 \mathrm{NF}$ is not enough to account for the differences. This is also true for the analyzing powers. The discrepancies generally become larger at higher incident beam energies. For other observables, the message is rather mixed. The addition of 3NFs sometimes improves the situation but sometimes also makes the agreement with the data worse. The effect of the Coulomb force, which has recently been taken into account [138], seems to be very small at all but the smallest angles for the elastic-scattering channel.

Many of these observables have been measured more than once at various laboratories for consistency checks. In most of the cases, the agreements are satisfactory, 


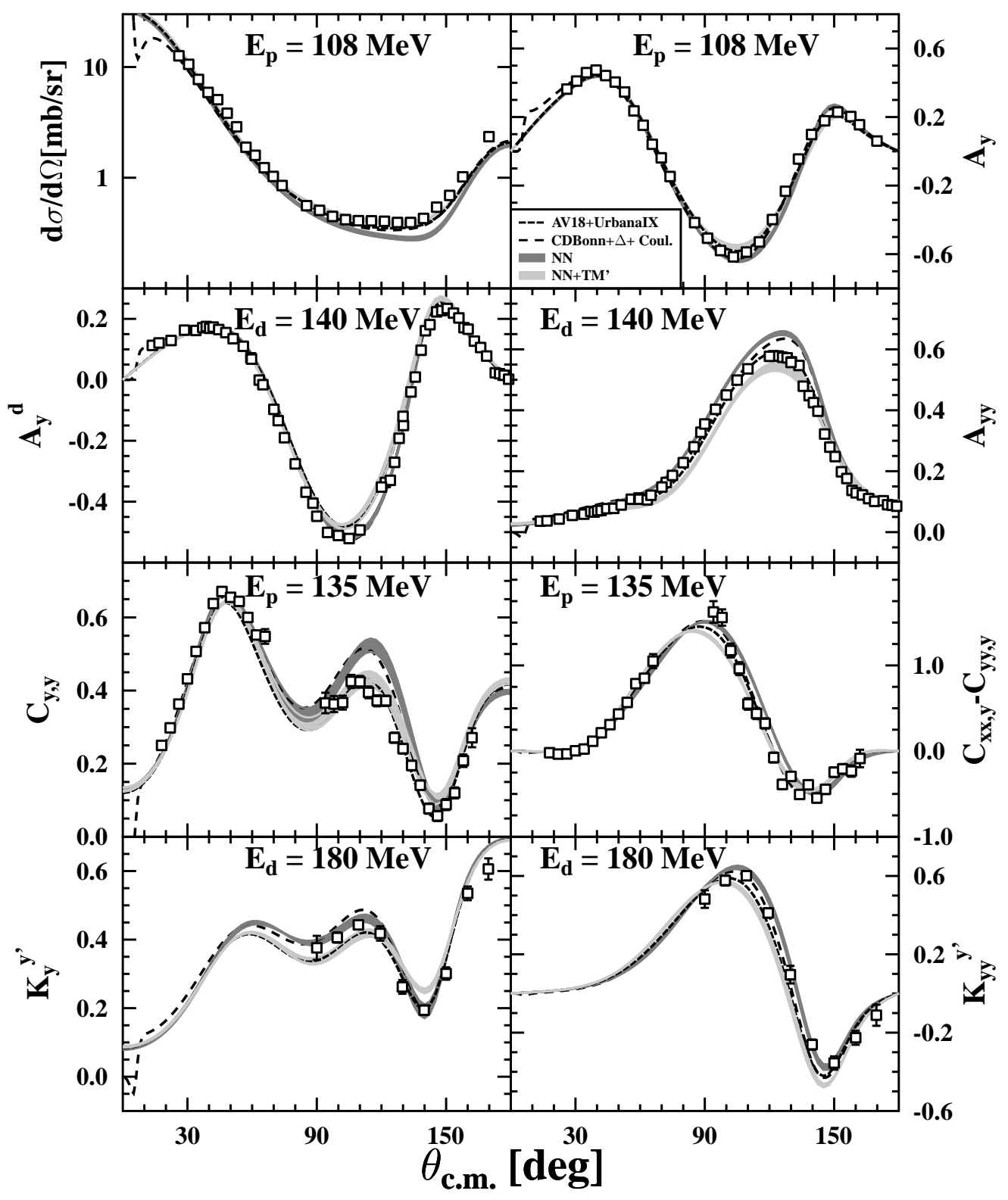

Figure 14. Differential cross sections and proton analyzing powers (top row), deuteron vector and tensor analyzing powers (second row), selected spin-correlation coefficients (third row) and selected spin-transfer coefficients (fourth row) for elastic scattering at an incident energy around $100 \mathrm{MeV} /$ nucleon. Errors are statistical only. The results of the calculations performed with several models including only two-nucleon (threenucleon TM') forces are shown with dark (light) grey bands while those with AV18 and UrbanaIX is presented by the short dashed line. The long dashed line represent the results of the calculations done by the Hanover-Lisbon group including the Coulombforce effects as well. Data are from Refs. [234, 236, 247, 257.

specially in the case of analyzing powers where the absolute normalization plays a minor role. In the case of the cross section, a major discrepancy has been observed between data sets taken at a beam energy of $135 \mathrm{MeV} /$ nucleon measured at KVI [234] and 


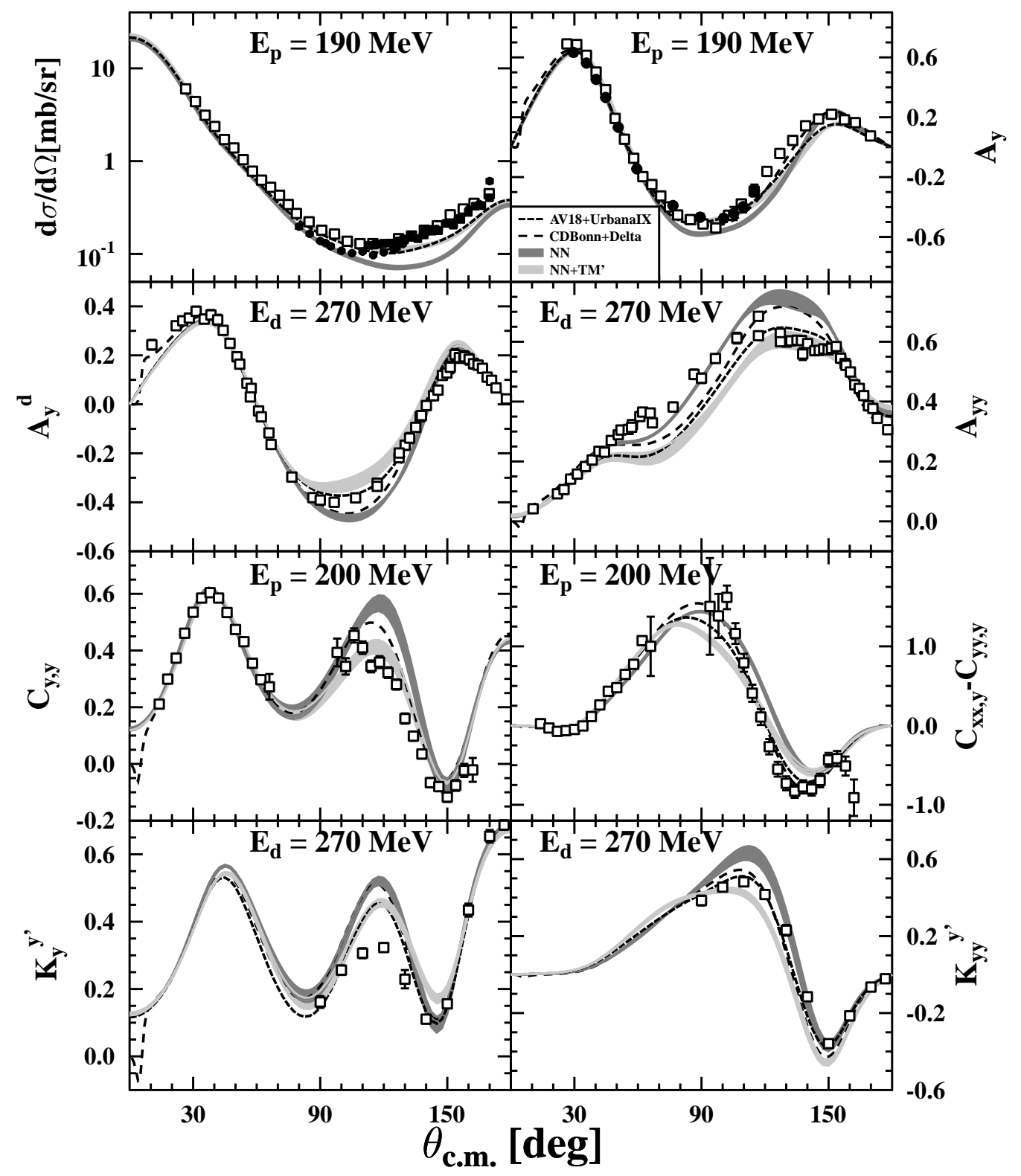

Figure 15. Same as Fig. 14 but for each observable at a higher energy. Data are from Refs. 231, 234, 247, 253, 255, 257.

RIKEN/RCNP [248]. Furthermore, data taken IUCF [257] agreed with the shape of the KVI data. Another measurement at KVI with a different setup has resulted in cross sections which are more compatible with the overall trend of the data at other energies and in disagreement with both earlier measurements [239].

The third observation is that for the break-up reaction at $65 \mathrm{MeV} /$ nucleon, the calculations with or without $3 \mathrm{NF}$ are in very close agreement with the data with a preference for the inclusion of the $3 \mathrm{NF}$ as was shown in a careful $\chi^{2}$ analysis [268]. With this reaction, one can also look at another variable, namely the relative energy of the two outgoing protons. It is intuitive and supported by the calculations that the 


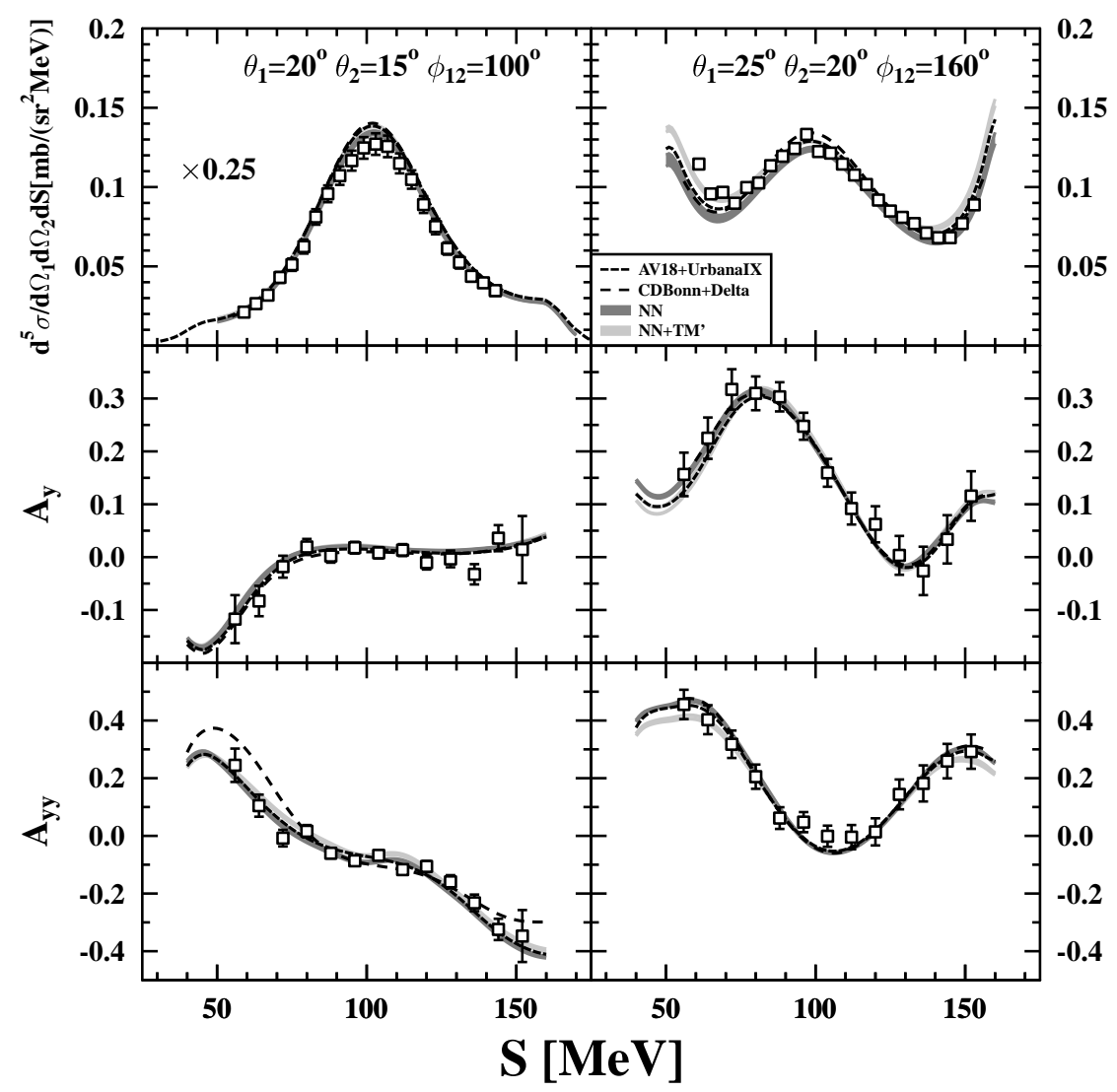

Figure 16. Differential cross sections (top tow), deuteron vector analyzing powers (second row), and deuteron tensor analyzing powers (third row) for the break-up reaction at two different kinematic configurations at an incident beam energy of $65 \mathrm{MeV} /$ nucleon. For the meaning of the lines, refer to Fig. 14.

Coulomb effect can be extremely large for the case where the relative energy of the outgoing protons is very small (see Fig. 18) [235]. This has been further pursued in a recently-performed experiment in Jülich where the opening angle between the two protons has been further reduced to $5^{\circ}[269$. At these very small angles, the Coulomb effect has even more dramatic features in the break-up cross sections.

Although the 3NF effects are generally small at $65 \mathrm{MeV} /$ nucleon, the situation changes with increasing beam energy. The results at $190 \mathrm{MeV} /$ nucleon clearly show that the $2 \mathrm{NF}$ is far from sufficient. Remarkably, the addition of a $3 \mathrm{NF}$ in some configurations, where the relative energy of the outgoing protons is small, makes the disagreements even larger (see Fig. 17). Possible missing ingredients which could contribute are relativistic effects (see section 3.1). This has now been included in a model calculation by Witała et al. [159]. The dashed-dotted curves in Fig. 17] show the results of these calculations. Although there are improvements in certain parts of the phase space, the effects are clearly not sufficient to describe the bulk of the data.

Note that in all the figures in this section with the exception of Fig. 18, only a phenomenological 3NF have been used which are "added" to the models which only include pair-wise NN interaction, and no results from the chiral EFT calculations are 


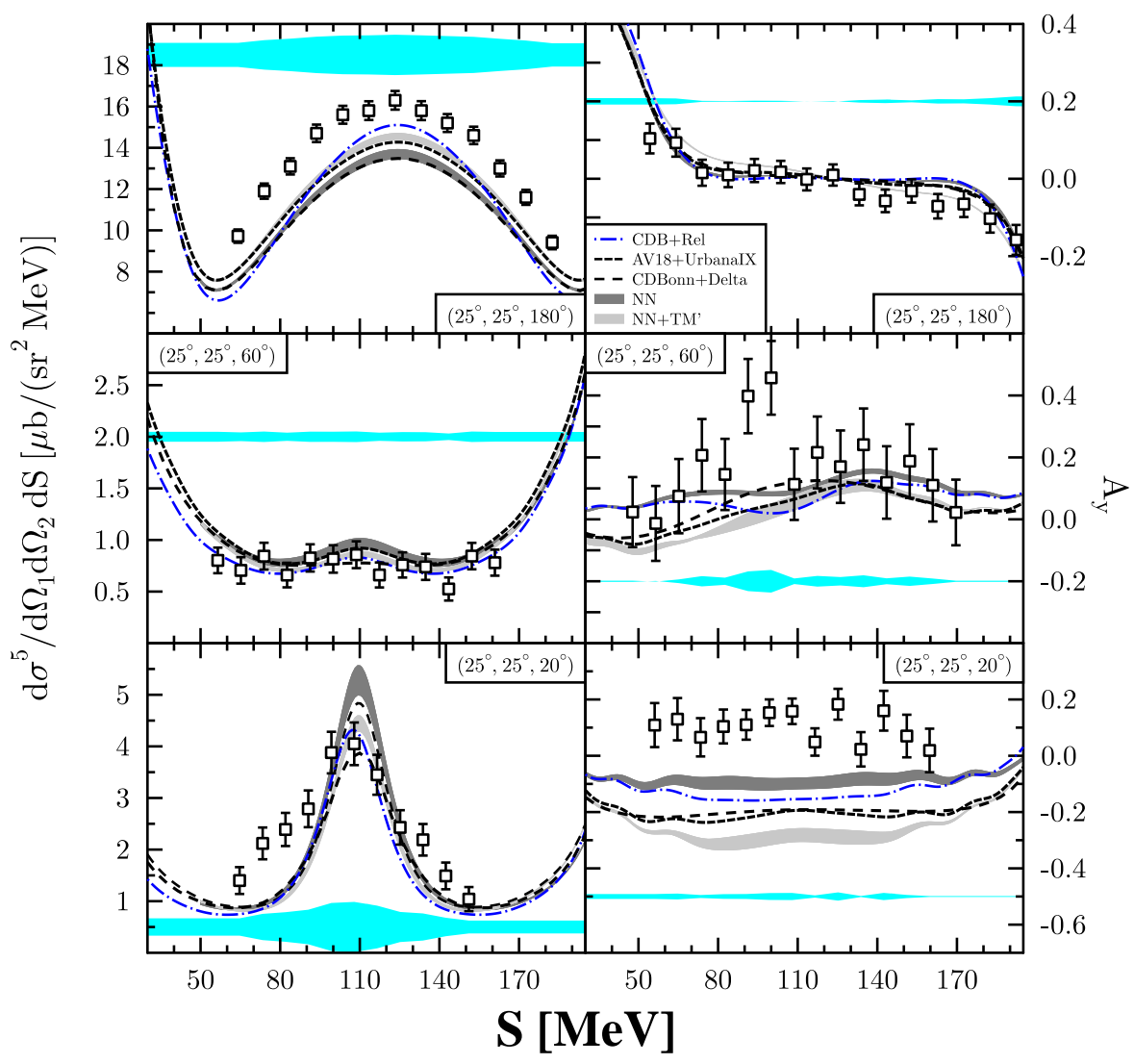

Figure 17. Differential cross sections (left column) and vector analyzing powers (right column) for the break-up reaction at three different kinematic configurations. Data are taken with a polarized proton beam of $190 \mathrm{MeV}$ 243,265. The horizontal bands show the $2 \sigma$ systematic uncertainty in the measurement of the observables. The dasheddotted line represents the results of a calculation including relativity. For the meaning of all other curves, refer to Fig. 14,

shown for intermediate energies. As mentioned in section 2, much progress has been made in the past decade in the framework of the chiral EFT. Presently, the calculations are showing a very good agreement with the data at low energies. As one increases the energy, the theoretical uncertainties at $\mathrm{N}^{2} \mathrm{LO}$ grow considerably. To illustrate this, two observables, namely cross section and analyzing power are shown as a function of scattering angle in Fig. 19 for 3 different energies. The model calculations shown in other figures agree quite well with the results of the chiral EFT calculations albeit within the large error bands. It is exactly because of these, sometimes, large theoretical uncertainties that they are not shown everywhere. We further emphasize that a rather large theoretical uncertainty for $A_{y}$ at low energy is a consequence of the extreme sensitivity of this observable to the NN triplet $\mathrm{P}$-waves which at $\mathrm{N}^{2} \mathrm{LO}$ are only described within a few percent. A considerably more precise description of these partial waves at $\mathrm{N}^{3} \mathrm{LO}$ leads to the same $A_{y}$-puzzle as observed for the phenomenological potential models, see Fig. 2 and the discussion of Ref. [270].

For our global analysis, all the data points have been collected along with the 


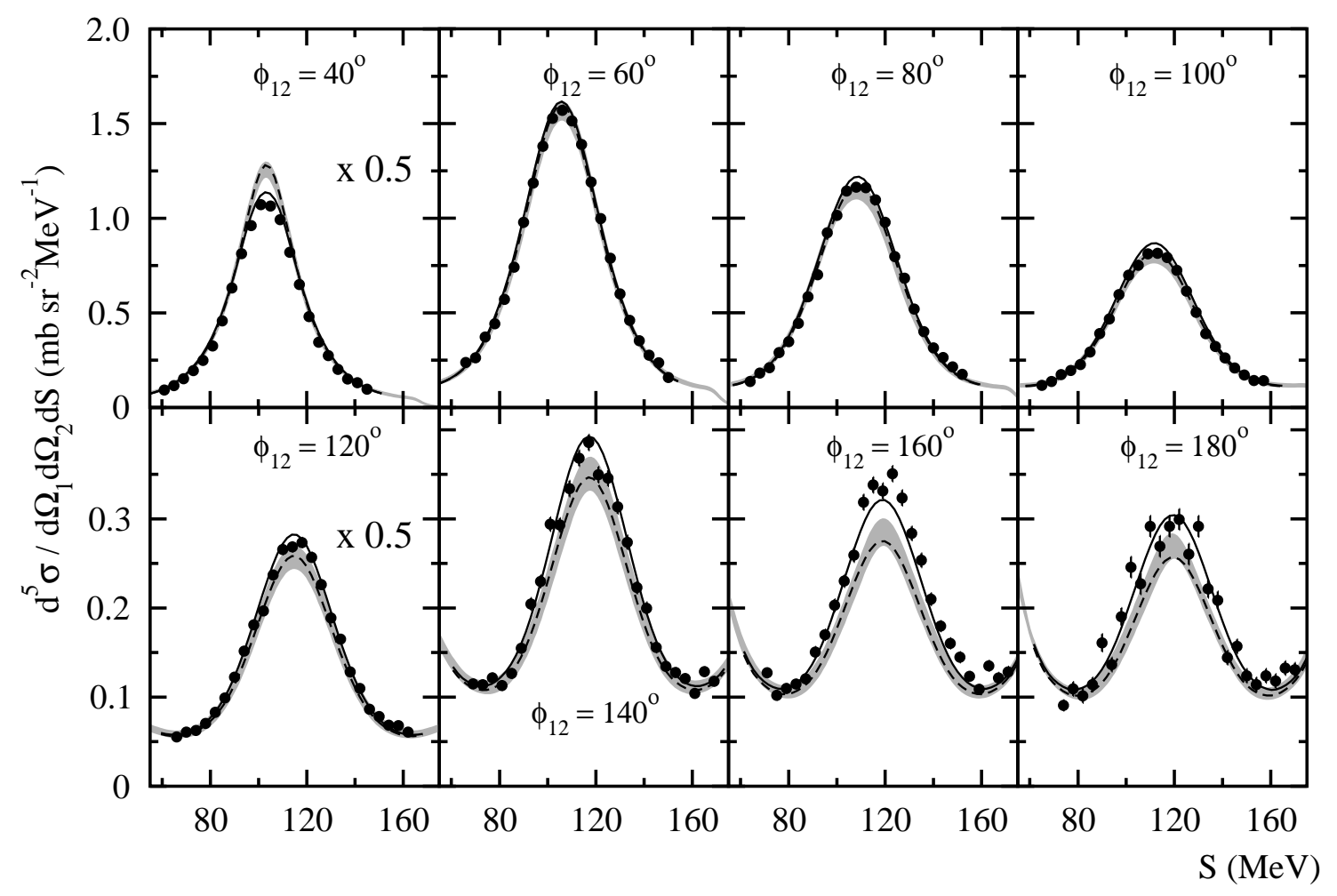

Figure 18. Results for the break-up cross sections for various coplanarity angles between the two outgoing protons $\left(\phi_{1}-\phi_{2}\right)$ as a function of the kinematical variable $S$, for an incident deuteron energy of $130 \mathrm{MeV}$ and the outgoing proton polar angles of $\left(15^{\circ}, 15^{\circ}\right)[235,268$. The bands show the results of the chiral EFT calculations at $\mathrm{N}^{2} \mathrm{LO}$. The curves are the results of the calculations of the Hanover-Lisbon group including the explicit $\Delta$ (dashed curve) and also including the Coulomb force (solid curve).

predictions of Hanover-Lisbon group. This group uses the CD-Bonn plus $\Delta$ potential based on the CD-Bonn refitted in order to accommodate the potential in a coupledchannel calculations including the $\Delta$ isobar. The reason for using this model is the inclusion of the Coulomb force effects which is very important in the comparison with data which are collected with charged-particle beams. The Coulomb effects are generally small but sizable at specific kinematics (small scattering angles for the elastic scattering and small relative energies of the outgoing protons in the case of the break-up reaction). The results of the predictions have, subsequently, been subtracted from the data for a certain observable. For the cross sections, the results are divided by the theoretical value to achieve relative deviations. For the polarizations observables, the absolute differences are investigated. The visualization of the $3 \mathrm{NF}$ effects shown below follows an idea of Meyer [275]. In the two-dimensional plots presented here (Figs. 20, 22, 23, and 24), these differences are shown for the case where the theory only includes $2 \mathrm{NF}$ (x-axis) and when it also includes 3NF (y-axis). All energies and all angles are included in the same figure. As a guide to understand the effects, three dashed lines have also 

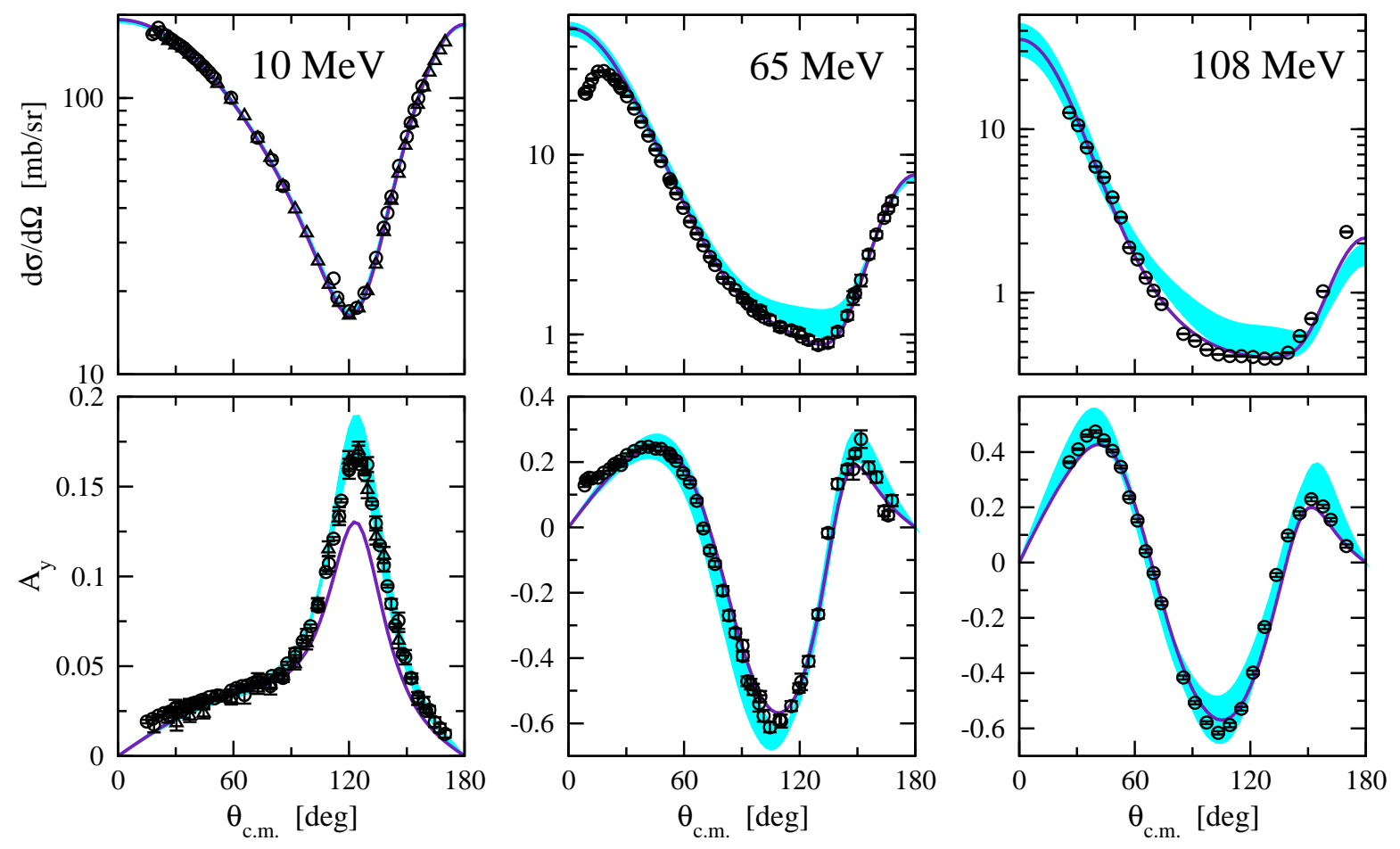

Figure 19. Results for the cross sections and analyzing powers as a function of centerof-mass scattering angle at three different incident energies. The bands are the results of the chiral EFT calculations at $\mathrm{N}^{2} \mathrm{LO}$. Also shown are the calculations based on the CD-Bonn potential combined with the Tucson-Melbourne 3NF. At $10 \mathrm{MeV}$, triangles refer to the neutron-deuteron data from Ref. [271] while circles are proton-deuteron data from Ref. [8, 272, 273] with the contribution due to the Coulomb interaction being subtracted. Proton-deuteron data at $65 \mathrm{MeV}$ and $108 \mathrm{MeV}$ are taken from Refs. [12,234,274] (color online).

been drawn in the figures. If the points lie on the vertical dashed line around 0 , one should conclude that the $2 \mathrm{NF}$ is already sufficient in the description of the data and the inclusion of the $3 \mathrm{NF}$ only deteriorates the agreement. If the points, on the other hand, lie on the diagonal dashed line, it means that the effect of $3 \mathrm{NF}$ is on average predicted to be negligible, irregardless of the agreement between the theory and the data. Finally, if the points lie on the horizontal dashed line around 0 , it means that the inclusion of the $3 \mathrm{NF}$ has accounted for the differences between data and models with only a $2 \mathrm{NF}$ as input. If the points lie around $(0,0)$, the data agree with the calculations including only $2 \mathrm{NF}$ and $3 \mathrm{NF}$ effects are shown to be small in the calculations and by the data.

To see trends in the data, the elastic-scattering cross sections and vector analyzing powers have been plotted with different shades (colors on-line) in Fig. 20, in the left panels, as a function of energy and in the right panels as a function of scattering angle in the center-of-mass. For the cross sections, a few observations can be made. The first one is that there are a number of points which lie in the lower left region of the origin. The bulk of these data points come from the high energy measurement at $250 \mathrm{MeV}$ (obscured in Fig. 20 by the black dots) [195] and the RIKEN measurement at 


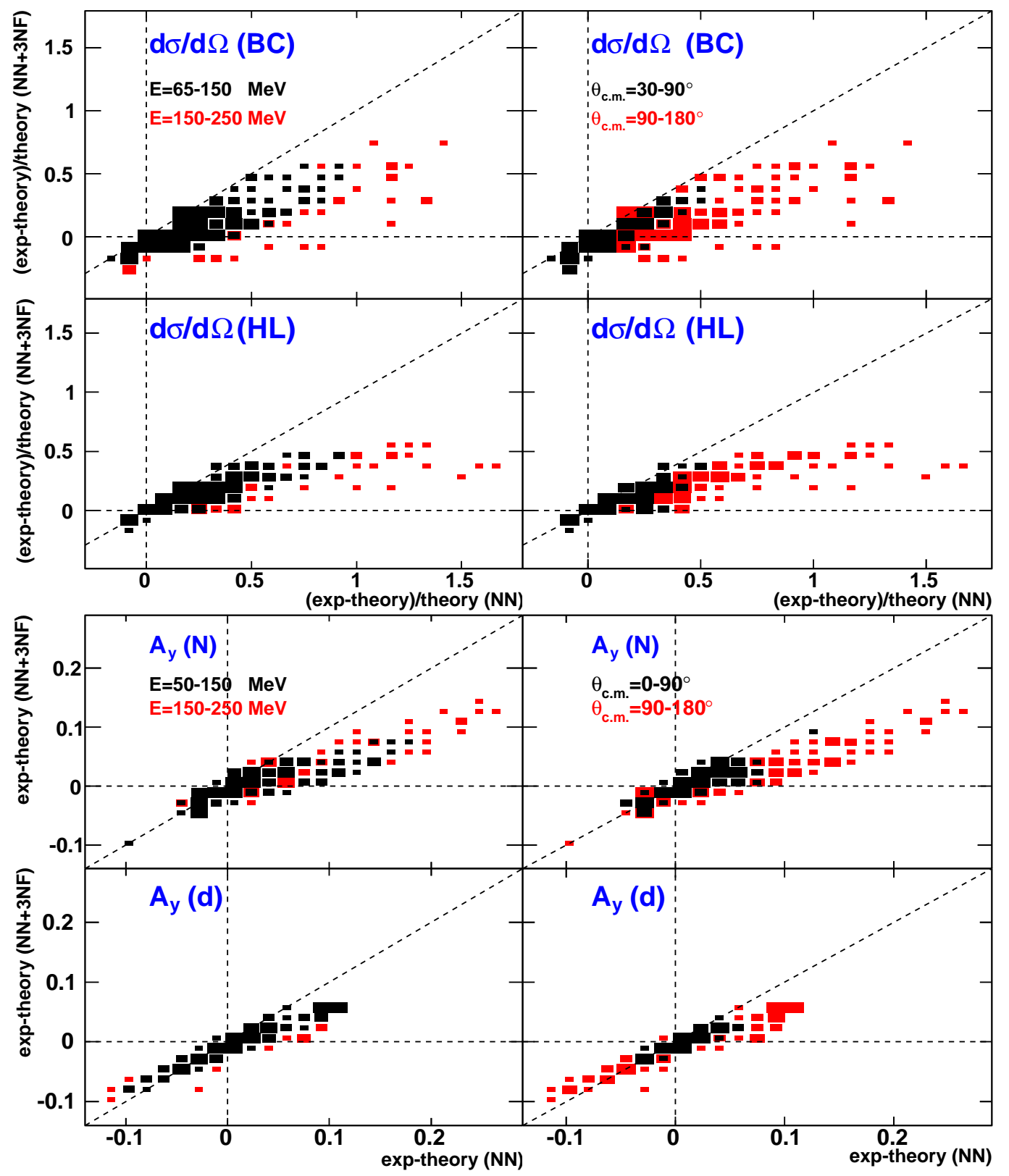

Figure 20. Results of the calculations are subtracted from all corresponding data points available in the literature for elastic scattering for the energy range of 50$250 \mathrm{MeV}$ and center-of-mass angles $\theta_{c . m .}>30^{\circ}$ and plotted as a (relative) difference between experimental data and calculations with only $2 \mathrm{NF}$ (x-axis) and with $3 \mathrm{NF}$ in addition (y-axis). The top four panels represent the relative differences for cross sections: on the left for two different energy ranges in two different shades (color online) and on the right for two different angle ranges in different shades. The label BC refers to a calculation from the Bochum-Cracow group based on the CD-Bonn two-nucleon potential and the TM' $3 \mathrm{NF}$. The label HL refers to a calculation from the HanoverLisbon group. A similar comparison is shown in the bottom four panels for the proton and deuteron vector analyzing powers. In this case, only the calculations of the HL have been used and $\theta_{c . m .}>8^{\circ}$ (color online). 
$135 \mathrm{MeV}$ [248], both at small scattering angles. Since Coulomb effects are very small at the angles chosen for this analysis and are properly taken into account in the calculations presented in the second row of the figure, these discrepancies with the calculations at the relatively small angles should be taken as a sign of normalization problems in the sense that the data lie below the calculations where one would expect a reasonable agreement due to the dominance of the $2 \mathrm{NF}$. Note that this problem is worse when one uses TM' 3NF (top panels) instead of the calculation using the $\Delta$ isobar (second row). The relative differences between these approaches are shown explicitly in Fig. 8. The same statement could also be made for the points which are on the opposite side of the origin but there, the signal is mixed with a genuine 3NF effect. The second observation is that the discrepancies become larger for higher energies and clearly become larger in the minimum of the cross section and for backward angles. The data are generally scattered between the diagonal line and the horizontal line. This implies that a $2 \mathrm{NF}$ is not enough to describe the data but that an additional $3 \mathrm{NF}$ within this model is also not sufficient to remedy the discrepancies. Other models of $3 \mathrm{NF}$ s have been examined as well and generally show the same pattern with the differences discussed when Fig. 8 was presented.

For the analyzing power, $A_{y}$, one can again observe that the discrepancy increases as the incident energy increases. Here, the largest discrepancies occur at scattering angles where the cross section is at its minimum. For the deuteron vector analyzing power, the discrepancies are generally smaller but extend to both sides of zero as opposed to the proton vector analyzing power which show differences which are generally on the positive side.

It is interesting to see in detail how the deviations for cross sections and vector analyzing powers behave as a function of scattering angle. These differences are shown in Fig. 21 for the case when only the two-nucleon forces are included in the calculations (left panels), and for the case where the effect of three-nucleon forces are also taken into account via the Hanover-Lisbon calculations (right panels). All three observables show a peak around the minimum of the cross section. The peak for the cross section vanishes only at the very backward angles whereas for the analyzing powers, the differences go through a zero when the cross section passes its minimum value and bend back towards zero at the very backward angles. The spread of the data around the peak position is due to the various energies that are included in the same figure. Generally, the higher the beam energy is, the larger the differences between data and predictions.

All other measured observables at intermediate energies are shown in Fig. 22, For the tensor analyzing powers, it is difficult to make a general statement for all observables. For $T_{21}$, most data points agree rather well with the calculations with and without the three-nucleon forces and largest discrepancies occur at backward angles. For $T_{20}$, the inclusion of three-nucleon forces seem to improve the agreement with the data slightly. For $T_{22}$, the discrepancies are not removed by the inclusion of three-nucleon forces. In fact, the contribution of the $3 \mathrm{NF}$ for this observable seems to be negligible. Also here, the largest discrepancies are observed at backward angles. The energy dependence of 


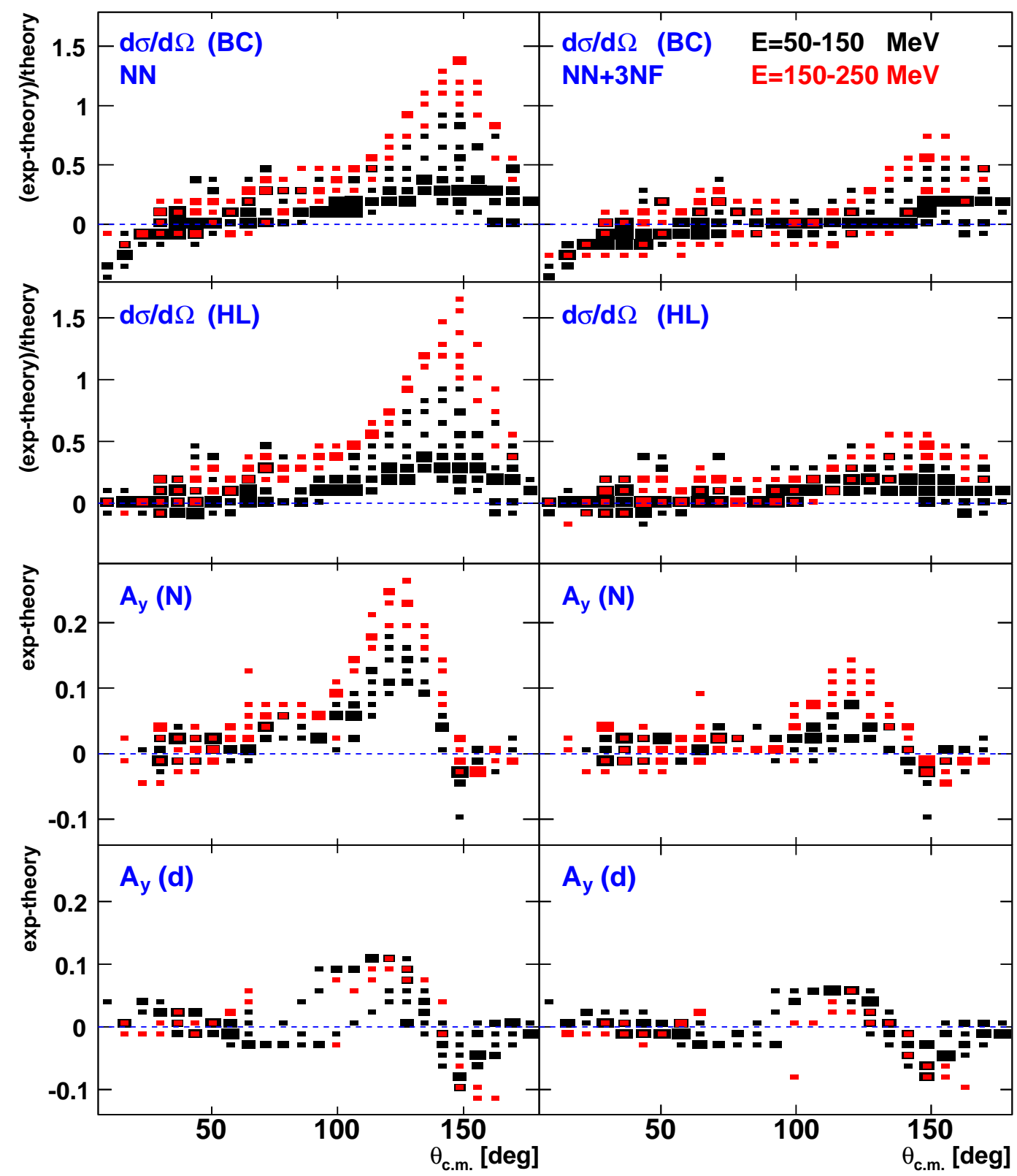

Figure 21. Cross-section (top four panels) and vector analyzing-power (third and fourth rows) differences for the case when only the two-nucleon forces are included in the calculations (left panels), and for the case where the effect of three-nucleon forces are also taken into account via the Hanover-Lisbon calculations (right panels) (color online). The label BC refers to calculations from the Bochum-Cracow group and the label HL to calculations from the Hanover-Lisbon group. In the top panels, the effects of the Coulomb force which becomes very strong below scattering angle of $25^{\circ}$ are observed as they are not included in the calculations by the BC group. For the analyzing powers, only calculations from the HL are shown.

the discrepancies is very small for tensor-analyzing observables.

A detailed study of the spin-transfer coefficients revealed similar behaviors among various groups of coefficients when comparing data with model calculations. Those 
Signatures of three-nucleon interactions in few-nucleon systems

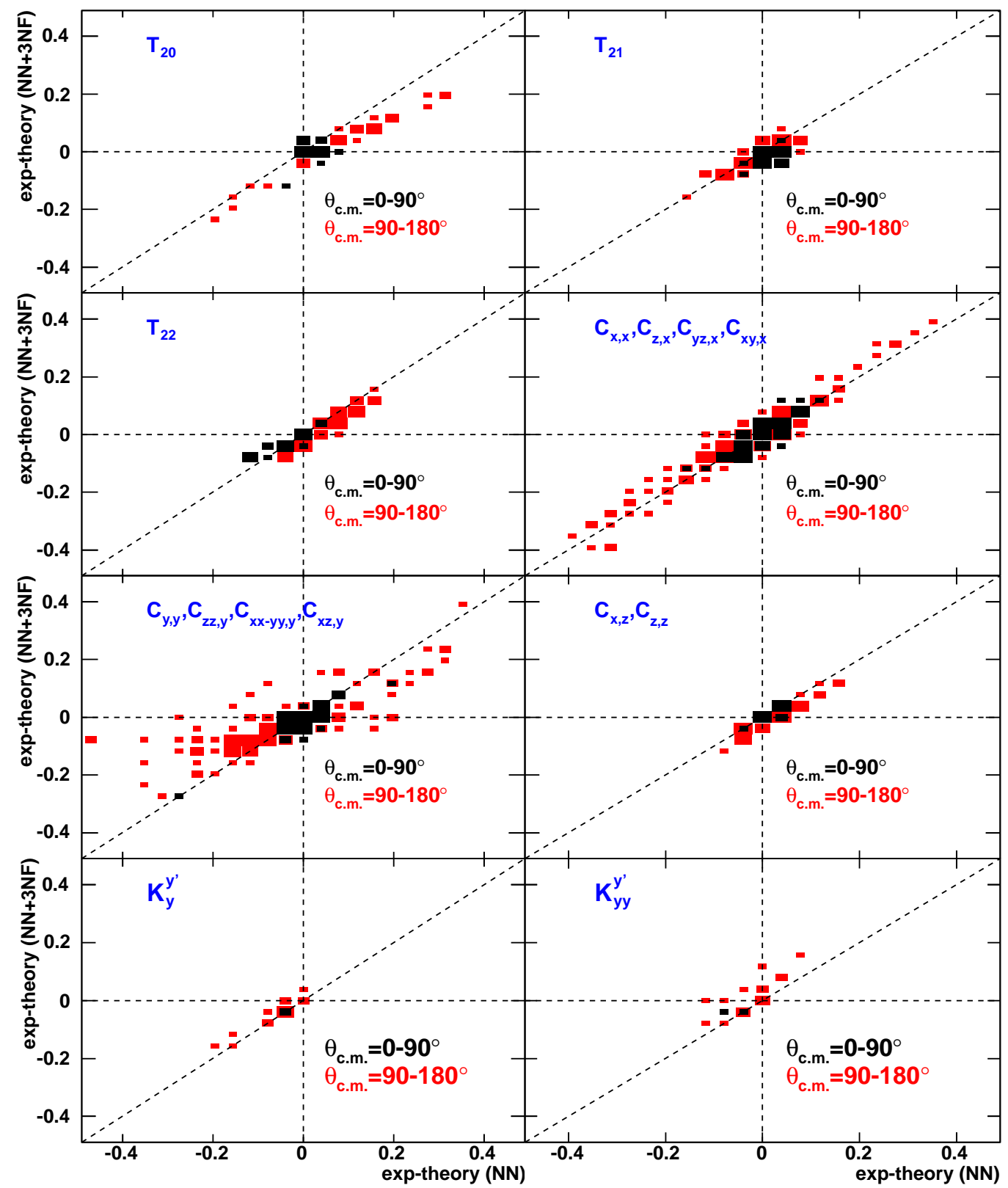

Figure 22. Results of the calculations are subtracted from all corresponding data points available in the literature for tensor analyzing powers, spin-transfer and spincorrelation coefficients in elastic scattering for the energy range of $50-250 \mathrm{MeV}$ and angular range of $15-175^{\circ}$ (not for all energies) and plotted as a difference between experimental data and calculations with only $2 \mathrm{NF}$ (x-axis) and with $3 \mathrm{NF}$ in addition (y-axis). The data points for all energies are mixed together and are only distinguished for two angular ranges with different shades (color online).

coefficients which showed similar trends were, therefore, combined in the global analysis as depicted in the middle panels of Fig. 22. The discrepancies are again very large, in particular for scattering angles larger than $90^{\circ}$. The different groups of coefficients, however, show different behaviors: $C_{x, z}$ and $C_{z, z}$ show good agreement with calculations and the effect of $3 \mathrm{NF}$ seem to be negligible in the calculations. For $C_{x, x}, C_{z, x}, C_{y z, x}$ 
and $C_{x y, x}$, there is a small effect due to the $3 \mathrm{NF}$ but the effect is in the wrong direction according to data. This is the opposite for $C_{y, y}, C_{z z, y}, C_{x x-y y, y}$, and $C_{x z, y}$ for which the addition of $3 \mathrm{NF}$ seems to bring the calculations much closer to the data. For these coefficients, the trends are the same for the two set of measurements at different energies.

For the $K_{y}^{y^{\prime}}$ and $K_{y y}^{y^{\prime}}$, the number of data points in the literature is limited due to the obvious limitation in the secondary scattering of the outgoing particles. What is in the literature for the intermediate energies (90 MeV/nucleon and $135 \mathrm{MeV} /$ nucleon) are plotted in the same way in the bottom row, as for the other observables in Fig. 22 . Here, one can see that the discrepancies are generally small and that the effect of the $3 \mathrm{NF}$ in the calculations is not significant (as could also be observed in Figs. 14 and 15). Given the small number of data points, it is difficult to draw strong conclusions for these observable. For the points which show some deviations from the calculations, it is clear that the addition of $3 \mathrm{NFs}$ is not sufficient.

Figure 23 shows the results of a global analysis for the break-up reaction at various energies (65, 130 and $190 \mathrm{MeV} /$ nucleon) for a large combination of angles. Here, the density of the data points is represented by the shade of grey. It is clearly seen that a large number of data points reside around $(0,0)$ indicating that the $2 \mathrm{NF}$ is already sufficient to describe the data reasonably well and that the effect of the $3 \mathrm{NF}$ is small. Many of these points stem, however, from the measurement taken at the lowest energy (65 MeV/nucleon). As the energy increases the length of the cluster around the diagonal line increases. As opposed to the elastic scattering channel, the deviations extend on both side of the origin: in some regions, the calculations overestimate the data and in others, they underestimate them. However, in almost all regions, the effect of the $3 \mathrm{NF}$ is not large and certainly not enough to remedy the differences. There is, however, a slight tendency towards repairing the deficiencies. The larger differences between the model predictions and the data come from kinematics for which the relative energy of the outgoing protons is large; see the top-right panel of Fig. 23 in which the relative difference between the data and the model calculations including $3 \mathrm{NF}$ is shown. The vector analyzing powers show a different behavior. For these observables, there are two bands: one on the diagonal and close to $(0,0)$ where the $3 \mathrm{NF}$ effects are small and a second band bending off from the diagonal indicating that the addition of the $3 \mathrm{NF}$ makes the agreement even worse. Further inspection of the data shows that these points belong to kinematics where the relative energy between the outgoing protons is small. This is illustrated in right panel of the figure for this observable. In the same panel, one observes several branches towards a relative energy of $0 \mathrm{MeV}$. The reason for the split is partly due to the fact that we have mixed data sets of two different beam energies (130 and $190 \mathrm{MeV} /$ nucleon). Another reason is that in the analysis of the data, one has chosen a coarse binning for the proton angles. In a hypothetical figure made with data points from many energies between 65 to $200 \mathrm{MeV}$ and all angles in a fine binning, one would then expect a cone shape with the base at $0 \mathrm{MeV}$ and the summit at the largest possible relative energy measured in the experiment. One should therefore look at the spin properties of the nuclear forces at small relative energies more carefully. 
The sample of data for $A_{y}^{d}$ is very small (only at $65 \mathrm{MeV} /$ nucleon) and the agreement seems to be reasonable, as shown in the third row of Fig. 23. A few data points which show large discrepancies occur at large relative energies. For the tensor analyzing power, the trends are very similar, namely that there is a good agreement between the predictions of the theoretical calculations including 3NFs (albeit small) and the data as shown in Fig. 24, For $A_{x y}$, a few data points show large discrepancies at larger relative energies. For the other two tensor analyzing powers, there seems to be no systematic pattern.

\subsection{How to proceed for $A=3$ systems?}

In the study of nuclear forces, the major effort was devoted to the nucleon-nucleon system during the last decades of the twentieth century. Two-nucleon force models were developed and fine tuned to the large data sets which became available. All physical observables are presently described with the help of these phenomenological potentials (CD-Bonn, AV18, NijmI, NijmII and Reid93) with a remarkable value of 1 for the $\chi^{2} /$ datum. With the good understanding of the long-range part of the potential, various attempts were made to do a model-independent analysis of all the data by bringing them together and performing a partial-wave analysis [89, 90]. The very large data set was brought successfully into very precise partial waves making the structure of the force very clear in terms of angular momenta.

It was only the precision in the two-nucleon sector and the technical possibility of doing exact calculations in the framework of Faddeev equations which made it possible to undertake the efforts and to study the more complicated systems starting with the threebody system. These studies initially took place at lower energies already showing very interesting features such as the the well-known $A_{y}$ puzzle. Also, the calculations proved to be easier and more practical at lower energies. In the 1990s, the focus was shifted towards intermediate energies and one tried to study various aspects in the three-body systems. This owed itself, to a large extent, to the fact that with large computational capabilities, one could perform exact calculations including large angular momenta for various observables up to energies below the pion-production threshold.

The interest in studying three-nucleon systems at intermediate energies required new detection systems and corresponding techniques; an effort which was taken up at several laboratories. The result of all the work in the past two decades is a database for the hadronic reactions in the three-nucleon system as shown in Fig. 12. The rich amount of available data show how well the field has matured and, as a result, our understanding of the nuclear forces has been significantly improved. The data in comparison with the state-of-the-art calculations also show (major) deficiencies in the models of the $3 \mathrm{NF}$. A natural question that now arises is whether it is possible to also parameterize the $3 \mathrm{NF}$ as it was done for $2 \mathrm{NF}$ in the past. One could argue that once $2 \mathrm{NF}$ and $3 \mathrm{NF}$ have the right parameters, any observable in the hadronic two- and three-nucleon sector will

be described by the models as it should since the parameters of the model would be 

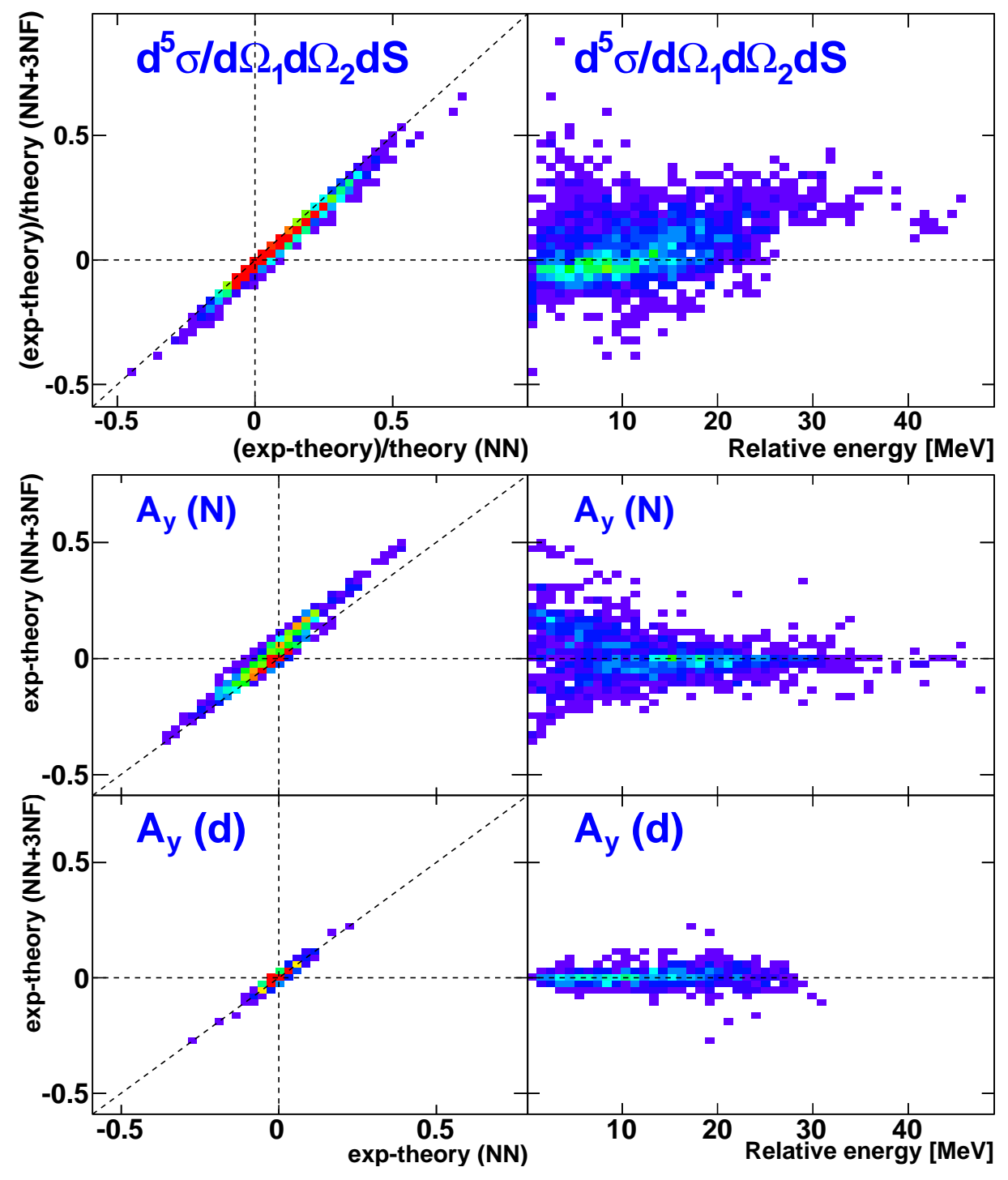

Figure 23. Results of the calculations are subtracted from all corresponding data points available in the literature for break-up reaction for the energy range of 65-190 $\mathrm{MeV}$ and various angle combinations and plotted, in the left panels, as a (relative) difference between experimental data and calculations with only $2 \mathrm{NF}$ (x-axis) and with $3 \mathrm{NF}$ in addition (y-axis). In the right panels, the differences between calculations including a $3 \mathrm{NF}$ and the data are shown as a function of the relative energy of the two outgoing protons. The top row shows the differences for cross sections, the second row, for the proton analyzing power, and the third row, for the deuteron vector analyzing power (color online).

fitted to the data. The size of the database and the accuracy of the data would then determine how good our understanding of the underlying structure of $3 \mathrm{NF}$ is. Recent analysis [243] shows that, once a large part of the phase space is covered, one has a tool to study specific aspects such as the isospin dependence of 3NFs.

Major investments for more experiments in these systems would require theoretical justifications. However, as was the case for the two-body system, the well-known 
Signatures of three-nucleon interactions in few-nucleon systems

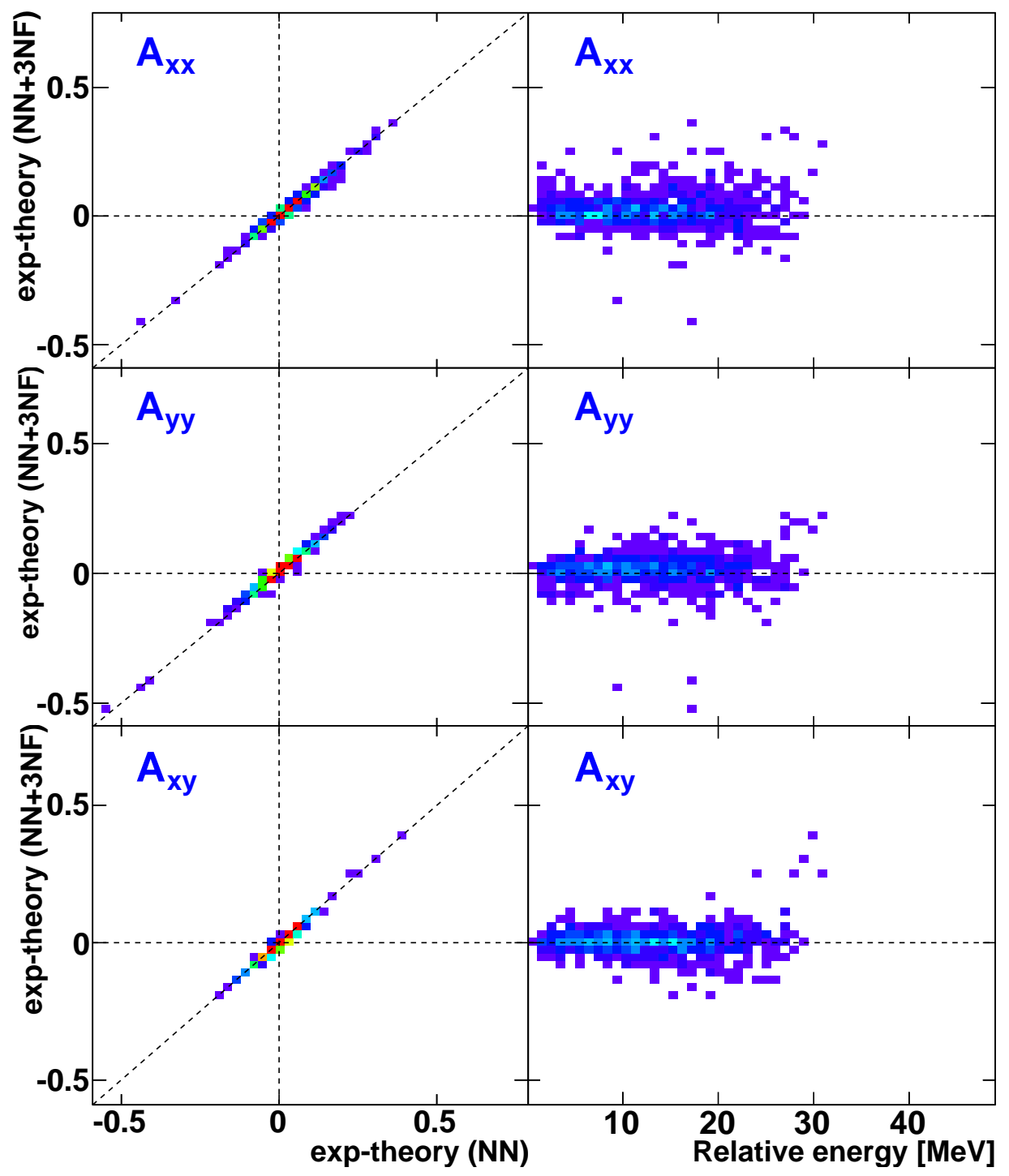

Figure 24. Results of the calculations are subtracted from all corresponding data points available in the literature for tensor analyzing powers of the protondeuteron break-up reaction. Presently, the database contains only a measurement taken at $65 \mathrm{MeV} /$ nucleon for various angular combinations. See Fig. 23 for a complete description of the plots (color online).

parameters of two-nucleon forces were changed (albeit at a fine level) with the latest experiments from IUCF [276] in the proton-proton system. The experimental and theoretical developments should, therefore, go hand in hand. Following the success in the two-body system, one obvious choice would be to perform a partial-wave analysis in the three-body system. The major challenge in performing this task is, however, the lack of an appropriate theoretical framework. A significant difference between the twobody and three-body systems is the very low-energy threshold of $2.2 \mathrm{MeV}$ which exists in the latter system. Already above this energy, an asymptotic three-body state needs to be formulated. This problem is not solved. Therefore, a PWA above this energy is technically impossible at the present moment. 
In the absence of a solid model-independent approach for the three-nucleon system at higher energies, one would have to rely on the models which exist in the literature and try to refine them with the help of the data. These refined models could then be used in performing calculations of observables in three-body scattering problems but also in larger systems described in the following sections. The question of whether one should perform more measurements should be addressed carefully. Figure 12 clearly shows that the database for the hadronic three-body reaction channels at intermediate energies is far from complete. The experimental situation for the elastic cross sections and analyzing powers seems to be, generally, well under control. The more exclusive spin observables, however, should be improved in the energy and angle range of the measurements. For the break-up channels, the database is even poorer and many observables are missing. Due to the difficulties in the measurements of these observables, and with the availability of exact three-body calculations and very fast computers, the design of any new experiment should be guided by detailed theoretical calculations which should help single out regions of phase space where all effects such $3 \mathrm{NF}$, Coulomb, relativity, etc. can be best studied. An example approach of this was performed by the Bochum-Cracow theory group in Ref. [277] in which the sensitivity to $3 \mathrm{NF}$ effects in the $\mathrm{N} d$ break-up reaction was provided as a guide for experimental activities. This was used as input for the design of a $4 \pi$ detection system, BINA, as shown in Fig. 10, In this context, analysis methods, such as the sampling technique, that provide a direct comparison between experiment and theory for the complex three-particle final state of the $\mathrm{N} d$ break-up reaction were applied very successfully [220,278]. Experiments that are designed to test explicitly the predictive power of the chiral EFT in the three-nucleon continuum are recommendable. An example of this is the PAX experiment at COSY [279] that will study the effects of current schemes for 3NFs that recently were implemented at third order in the chiral EFT calculations and diagrams appearing at fourth order. Aside from new measurements, there are also measurements in the literature which require a re-measurement or re-analysis of the data due to disagreements with each other or due to internal inconsistencies. There are also measurements which have been performed with limited statistics or limited coverage of kinematical variables. New measurements to address these problems should also be performed. These measurements can nowadays only take place at RCNP (and to a lesser extent at RIKEN) and Jülich at intermediate energies.

\section{Three-nucleon forces and $A=4$ systems}

\subsection{Are four-nucleon forces important?}

Clearly, the $3 \mathrm{~N}$ system is the cleanest laboratory to study $3 \mathrm{NF}$ s since by construction only NN and $3 \mathrm{~N}$ forces can contribute. The sensitivity of $3 \mathrm{~N}$ observables is however limited. We have seen that one has to go to intermediate energies and to search very specific configurations to identify signatures of $3 \mathrm{NFs}$ in $3 \mathrm{~N}$ observables that are not 
linked to the ${ }^{3} \mathrm{H}$ binding energy. Three-nucleon bound states with other spin/isospin configurations do not exist, so that studies of the spin-isospin structure of $3 \mathrm{~N}$ force can only be performed based on the $3 \mathrm{~N}$ scattering data in the three-body systems. In view of these constraints, it appears promising to study $3 \mathrm{NF}$ effects in four-nucleon $(4 \mathrm{~N})$ systems. It is the simplest nuclear system that supports resonance structures besides the ${ }^{4} \mathrm{He}$ bound state. It is conceivable that the resonance energies are similarly sensitive to $3 \mathrm{NF}$ contributions as the 3N binding energy. Since resonances of different spin and isospin exist, it should become possible to study the spin and isospin dependence of the $3 \mathrm{NF}$ in the very low energy regime.

Obviously, to achieve this goal, it is necessary to control the size of four-nucleon force $(4 \mathrm{NF}$ ) contributions. Although first studies of selected $4 \mathrm{NF}$ contributions appeared in the 1980s [280,281], not much is known about their size. The general expectation is that the $4 \mathrm{NF}$ contributions at least to ${ }^{4} \mathrm{He}$ are small. Mostly, this expectation is based on the observation that the ${ }^{3} \mathrm{H}$ and ${ }^{4} \mathrm{He}$ binding energy are almost linearly correlated (Tjon line, [282, 283]) and that extrapolating the results of phenomenological model calculations almost hits the experimental 3N/4N binding energies (see footnote on page 16). Also the rather successful description of spectra and binding energies of light nuclei based on $\mathrm{NN}$ and $3 \mathrm{~N}$ forces [154,284] is generally seen as support for the assumption that $4 \mathrm{NFs}$ are negligible.

More quantitative is the analysis of the Hanover-Lisbon group [285]. Assuming that the most important $4 \mathrm{NF}$ contribution is due to intermediate $\Delta$ excitations of twonucleons interacting with the two others, they were able to extract the $4 \mathrm{NF}$ contribution from their calculations with explicit $\Delta$ degrees of freedom. Both for various low-energy scattering observables and for the ${ }^{4} \mathrm{He}$ bound state, they found the $4 \mathrm{NF}$ effects are not significant. For example, the contribution to the binding energy of ${ }^{4} \mathrm{He}$ is $170 \mathrm{keV}$. This is approximately $0.2 \%$ of the potential energy and can be considered as negligible.

In the framework of chiral EFT, the size of $4 \mathrm{~N}$ forces can be estimated based on the underlying power counting [162. The first $4 \mathrm{NF}$ contribution appears at $\mathrm{N}^{3} \mathrm{LO}$. Therefore, the contribution can be expected to be less important than the leading $3 \mathrm{NF}$. It is possible to check this expectation from power counting and to perform complete N3LO calculations in future, since the leading $4 \mathrm{NF}$ has been worked out in Refs. [286, 287]. In addition to the well-known long-ranged pion-exchange contributions which were already discussed in the literature [280,281], one finds that there are shorter-ranged contributions directly linked to the short-range part of the NN interaction. In this way, one obtains a consistent set of $\mathrm{NN}, 3 \mathrm{~N}$ and $4 \mathrm{~N}$ forces. Of utmost practical importance is that the leading $4 \mathrm{NF}$ is completely determined by parameters that appear in the NN interaction. $\mathrm{No} 4 \mathrm{~N}$ datum is required to determine this $4 \mathrm{NF}$ quantitatively. In contrast to the leading 3NF, which is driven by LECs saturated by the $\Delta$, no such LECs appear in the leading 4NF. In this sense, chiral EFT predicts a completely different 4NF than the one taken into account by the Hanover-Lisbon group. It is, therefore, interesting to calculate the contribution of 4 NFs to nuclear systems. This has recently been achieved based on a perturbative calculation of the $4 \mathrm{NF}$ contribution to the ${ }^{4} \mathrm{He}$ binding energy [288]. It 
was found that for the chiral interactions of [88], the $4 \mathrm{NF}$ contribution is of the order of $300 \mathrm{keV}$. Again, this is only $0.3 \%$ of the potential energy. It is much smaller than the typical contribution of the $3 \mathrm{NF}$ to the ${ }^{4} \mathrm{He}$ binding energy of $2-4 \mathrm{MeV}$ [163] and probably negligible for most studies of the $4 \mathrm{~N}$ system. Note, however, that for large cutoffs and for phenomenological interactions, the contributions were somewhat larger pointing to a possible scheme dependence of the importance of $4 \mathrm{NFs}$.

In summary, the preliminary results do not indicate that 4 NFs give significant contributions for the standard low cutoff chiral interactions. It is not clear whether this is true for phenomenological interactions since the estimates based on chiral $4 \mathrm{NF}$ are not completely negligible. We, however, stress that in all cases the contribution of the $4 \mathrm{NF}$ is significantly less important than the contribution of the leading 3NFs. Based on this last insight, we can expect that calculations without $4 \mathrm{NF}$ will be sufficient to test the contribution of the leading $3 \mathrm{NFs}$ in $4 \mathrm{~N}$ systems.

\subsection{Four-nucleon systems at low energies}

The $4 \mathrm{~N}$ systems promise to provide valuable information on nuclear dynamics. It is not only a simple extension of $3 \mathrm{~N}$ dynamics to a slightly more complex system since it is the first system that supports a whole spectrum including the ${ }^{4} \mathrm{He}$ bound state and several resonance structures for which data are available in different isospin channels [289]. Assuming the energies of the resonances are similarly sensitive to nuclear interactions as the binding energies, scattering observables should significantly depend on 3NFs even at very low energies of a few $\mathrm{MeV}$. Because the states have different spins and isospins, this should open the possibility to study the spin and isospin dependence of the 3NFs in the low-energy regime where a fast convergence of the chiral expansion can be expected. Obviously, the $4 \mathrm{~N}$ system can only be a unique laboratory to study 3NFs if theoretical approaches are available that enable one to solve the $4 \mathrm{~N}$ problem with the same accuracy and reliability as it is nowadays possible for the $3 \mathrm{~N}$ problem.

Such methods have been devised in recent years. In the following, we would like to summarize briefly the current status of this development. In order to be concise, we will focus on methods that have shown the reliability for modern nuclear interactions including all the complexity. Pioneering calculations, which unfortunately needed uncontrolled approximations, are summarized in Ref. [289]. We stress that such approaches were important milestones for the development of the methods but are not suitable for studies of subtle contributions of 3NFs.

The solution of the ${ }^{4} \mathrm{He}$ bound state problem has become standard by now. Many methods have been devised and have been benchmarked for rather sophisticated NN interactions (see [290] and references therein). Also when 3NFs are included, there are several methods that have proven their reliability, e.g. the Green's Function Monte Carlo (GFMC) approach [4], the no-core shell model (NCSM) [291], the hyperspherical harmonics expansion [292], solutions of Faddeev-Yakubovsky equations in momentum [283, 285] and configuration space [293,294] have been used to obtain solutions for a 
wide class of $\mathrm{NN}$ and $3 \mathrm{~N}$ interactions.

Unfortunately, the information on $3 \mathrm{NFs}$ which can be extracted from the ${ }^{4} \mathrm{He}$ bound state is limited due to a strong correlation between the $3 \mathrm{~N}$ and $4 \mathrm{~N}$ binding energies called the Tjon line (which, actually represents a band) [282]. As a consequence, although the $3 \mathrm{NF}$ contribution to the ${ }^{4} \mathrm{He}$ binding energy is much larger than the $4 \mathrm{NF}$ contribution, the changes of the binding energy are rather subtle when the ${ }^{3} \mathrm{H}$ binding energy is kept constant. Therefore, it is not completely clear that these changes can be disentangled from effects of $4 \mathrm{NFs}$. Despite this, since two data points are required to fix the two unknown parameters of the $3 \mathrm{NF}$, the ${ }^{4} \mathrm{He}$ binding has been used to determine the $c_{D}$ and $c_{E}$ parameters of the leading chiral $3 \mathrm{NF}$ [295]. It turned out that it is not always possible to find a unique set of $3 \mathrm{NF}$ parameters. These extractions were nevertheless valuable to confirm the effects of $3 \mathrm{NFs}$ for systems with $A>4$ (see next section). In the course of such investigations, it was realized that the radius of ${ }^{4} \mathrm{He}$ is also sensitive to the $c_{D}$ and $c_{E}$ parameters and can be used to fix their values in a probably more reliable way [284]. All these investigations showed, however, that the ${ }^{4} \mathrm{He}$ bound state is not a good laboratory to study the spin/isospin dependence of 3NFs. This can be traced back to the dominance of $J^{\pi}=1 / 2^{+} 3 \mathrm{~N}$ partial wave states in the ${ }^{4} \mathrm{He}$ wave function that are very similar for the ${ }^{3} \mathrm{H}$ bound state.

Much more promising is the solution of the $4 \mathrm{~N}$ continuum problem. Although considerable progress has been made over the last 10 years, this problem is still not completely solved. Reliable theoretical results have only been obtained below the socalled three-body break-up threshold namely the energy where the system can break-up in two nucleons and the deuteron or in four nucleons. A first pioneering study below break-up threshold based on Yakubovsky equations was already reported in [296] but was only continued by other groups later.

The simplest system for which data exist is the neutron- ${ }^{3} \mathrm{H}$ system. Nevertheless, it shows the most important features of the $4 \mathrm{~N}$ system while no Coulomb interaction needs to be taken into account. At the same time, the threshold for the first break-up is at rather high energies (at approximately $8.3 \mathrm{MeV}$ neutron laboratory energy) and data for the energy dependence of the total cross section and some differential cross sections exist.

Obviously, the most basic observable is the zero energy cross section, which is conventionally parametrized in terms of the scattering lengths for spin-0 and spin1 neutron- ${ }^{3} \mathrm{H}$ states $\left(a_{0}\right.$ and $a_{1}$ ). The scattering lengths have first been studied theoretically in detail in [297,298]. Calculations based on NN interactions overpredict the zero energy cross section and the scattering lengths considerably. This failure could be traced back to the underbinding of ${ }^{3} \mathrm{H}$ predicted by the NN models. Again, there is a correlation between the scattering lengths and the few-body binding energies [297] and the experimental data are fairly well reproduced once the binding energies are reproduced. In this context, the calculations indicate that the scattering lengths are not completely consistently extracted from the two experimental results, the zero energy cross section and the so-called coherent scattering length [294]. Such an inconsistency 


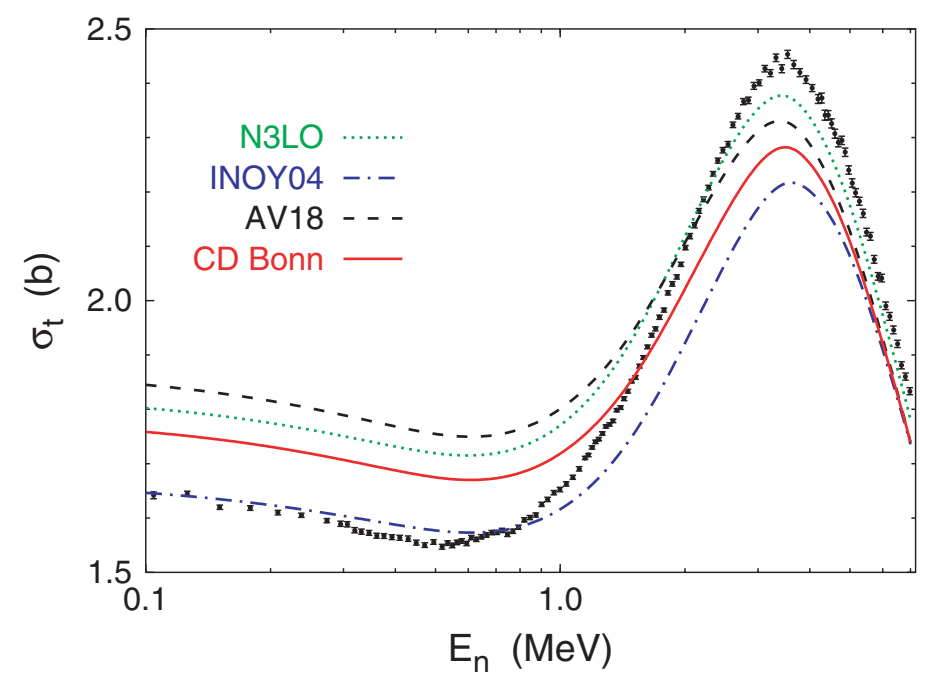

Figure 25. Total neutron- ${ }^{3} \mathrm{H}$ cross section $\sigma_{t}$ as a function of the neutron laboratory energy $\left(E_{n}\right)$. The calculations are from Ref. [302]. Data are from Ref. 304] (color online). Reprinted with permission from 302. Copyright (2007) by the American Physical Society.

calls for an independent measurements of these fundamental quantities.

The total cross section for neutron- ${ }^{3} \mathrm{H}$ scattering already has an interesting energy dependence. It has a broad peak at approximately $3 \mathrm{MeV}$ which is generated by several P-wave resonances [289. This energy dependence has been the focus of the early realistic calculations [294, 298]. While first calculations with simple NN interactions or with approximated NN interactions showed agreement with the data [299, 300], this was not the case for the complete calculations. This could finally be traced back to the simplifications in the first calculations 301] so that it is clear by now that the height of the peak is significantly underpredicted by realistic calculations (see Fig. 25). Studies including the $3 \mathrm{NFs}$, various NN interactions or explicit $\Delta$ show that this failure is not correlated to the inaccurate description of the ${ }^{4} \mathrm{He}$ binding energy and cannot be remedied by inclusion of standard 3NFs [285, 294, 302]. Therefore, the energy dependence of the total neutron- ${ }^{3} \mathrm{H}$ cross section already constitutes a first puzzle in the $4 \mathrm{~N}$ continuum calling for more systematic studies including more complex three-nucleon forces. Indeed a first calculation including all topologies of the leading chiral 3NF (see upper part of Fig. (7) gives a much better description of the data [303].

Since neutron $-{ }^{3} \mathrm{H}$ experiments are difficult, the proton- ${ }^{3} \mathrm{He}$ mirror system has attracted a lot of attention in spite of the additional complications due to the Coulomb interaction of the protons. The proper inclusion of Coulomb effects was first achieved in configuration space approaches, where the proper boundary conditions can be implemented for two cluster states [305, 306]. Later, Coulomb forces were also implemented in momentum space [307].

Based on these advances, it was realized that, although the differential cross section of low-energy $p{ }^{3} \mathrm{He}$ scattering is described fairly well by phenomenological interactions, 


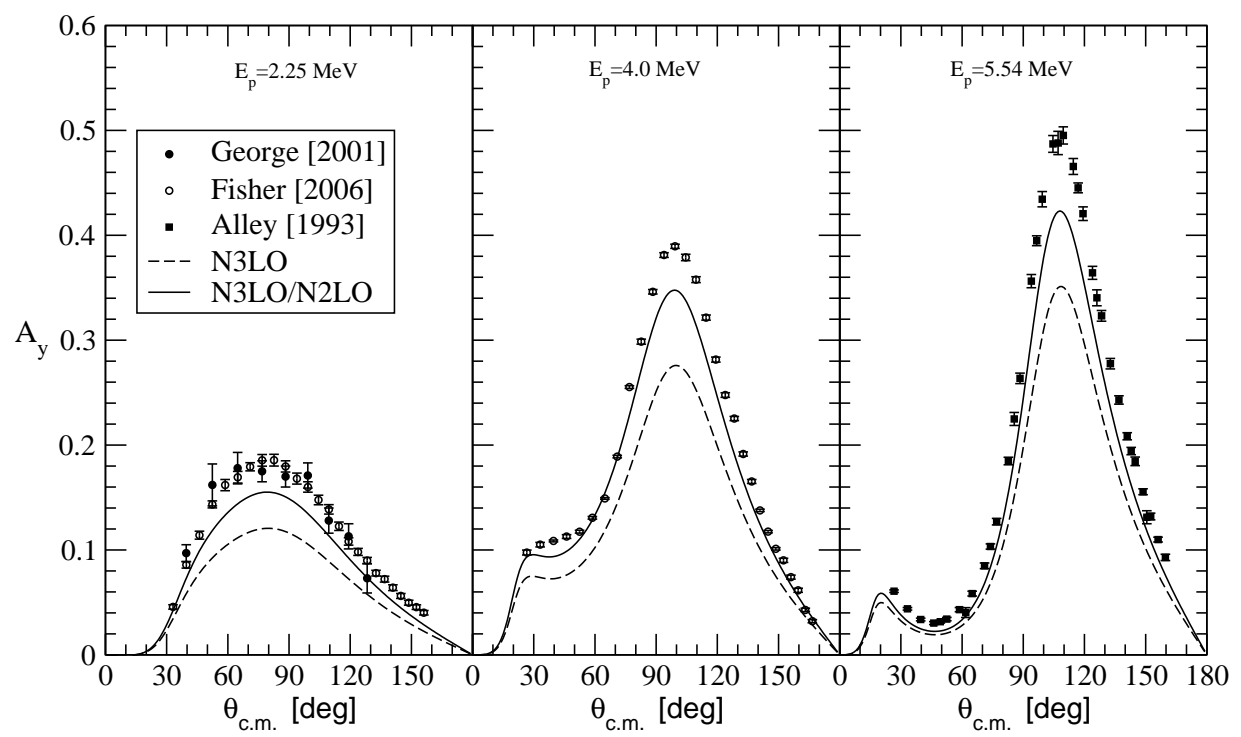

Figure 26. Proton $A_{y}$ in $p^{-3}$ He scattering at three different energies compared to the preliminary calculations of Ref. 309,310 . The solid (dashed) lines are predictions based on the chiral interaction of Ref. [87 with (without) 3NFs. Data are from Refs. [305, 308, 311.

there is a sizable underprediction of the proton analyzing powers $\left(A_{y}\right)$ [305, 308]. The size of this failure is much more significant than in the $3 \mathrm{~N}$ system. In the $3 \mathrm{~N}$ system, the absolute size of $A_{y}$ is only a few percent and the absolute deviations are of the order of a percent. In contrast, in the $4 \mathrm{~N}$ system, the magnitude of $A_{y}$ is much larger so that the absolute deviations are as high as $15 \%$. In view of the size of this deviation, it is astonishing that the predictions are not significantly dependent on NN forces [307] and the standard 3NFs are unable to remedy the problem [305,308]. However, recently a preliminary study of the Pisa group showed that the leading chiral 3NF, properly adjusted to the ${ }^{3} \mathrm{He}$ and ${ }^{4} \mathrm{He}$ binding energies, significantly improves the description on $A_{y}$ [309], see Fig. 26. At the same time, the chiral 3NF does not distort the favorable description of other observables. This would probably be the most prominent signature of the topologies of chiral 3NFs observed so far. Interestingly, the same force is not able to improve the description of $A_{y}$ in proton ${ }^{2} \mathrm{H}$ scattering (see Fig. 2) leaving room for the action of higher order $3 \mathrm{NFs}$.

Besides the simplest elastic processes, there are also several possible reactions starting, e.g., with two deuterons in the initial state exemplifying the richness of the four-nucleon system for studies of nuclear reactions:

(i) Elastic channel: $d+d \longrightarrow d+d$;

(ii) Neutron-transfer channel: $d+d \longrightarrow p+t$;

(iii) Proton-transfer channel: $d+d \longrightarrow n+{ }^{3} \mathrm{He}$;

(iv) Three-body final-state break-up: $d+d \longrightarrow p+n+d$; 
(v) Four-body final-state break-up: $d+d \longrightarrow p+n+p+n$.

Studying the processes listed above with the same rigor becomes very involved once three- and four-body channels are open. In configuration space, such channels have a very complicated asymptotic behavior and in momentum space the singularities of the integral kernels become cumbersome. Below the three- and four-body thresholds, $d d$ scattering has already been studied in [300] where it is pointed out that also NN Pwaves give important contributions. Advanced calculations of $d d$ scattering are reported in [285,312]. This study also includes 3NFs due to $\Delta$ isobar contributions. Generally, the calculations agree well with experiment for elastic scattering and rearrangement to $p+{ }^{3} \mathrm{H}$ and $n+{ }^{3} \mathrm{He}$. But there are some interesting discrepancies of theory and data for polarization observables. The contribution of the 3NFs induced by $\Delta \mathrm{s}$ is usually small. It will be interesting to check this for chiral 3NFs that have helped to improve the situation for elastic $n-{ }^{3} \mathrm{H}$ scattering as discussed above.

A complete overview of the different $4 \mathrm{~N}$ reactions at low energy has been given by Hofmann and Hale [313. The experimental data have been used for an R-matrix analysis, which is compared to resonating group model (RGM) calculations. Based on the RGM calculations, it is argued that 3NFs give important contributions to lowenergy $4 \mathrm{~N}$ reactions. By means of the R-matrix analysis, Hofmann and Hale identify observables that are specifically well suited to further constrain phase shifts and that should be measured with higher priority. Given the interesting sensitivity of many of the $4 \mathrm{~N}$ observables to $3 \mathrm{NFs}$ discussed above such measurements will be important to advance our understanding of $3 \mathrm{NFs}$.

\subsection{Four-nucleon systems at intermediate energies}

In this section, the recent developments made in the four-nucleon systems at intermediate energies will be discussed. Contrary to the low-energy regime, where many calculations have been performed (see last section), there are presently no calculations done for scattering observables at these energies. This is partly due to the fact that the deuteron break-up threshold is very low. The main problem of the calculations is the treatment of break-up configurations. Already for the three-body break-up channels, this becomes very cumbersome in configuration space calculations, which is reflected in a difficult singularity structure in momentum space. Therefore, Uzu, Kamada, and Koike tried to avoid the singularities completely by performing the calculations for complex energies and analytically continuing to the real axis [314]. They demonstrated that this approach is feasible and may lead to reliable results also for energies well above threebody break-up. It is, however, difficult numerically to ensure the accuracies required for such a continuation. Especially, when the energies are close to the real axis, it is difficult to perform the $4 \mathrm{~N}$ calculations with such a high accuracy. Therefore, so far no realistic calculations have been performed in this energy range. Instead, crude approximations have been used to take $4 \mathrm{~N}$ rescattering at high energies into account mainly to estimate the size of possible effects (see e.g. [315 317]). For another recent proposal to simplify 

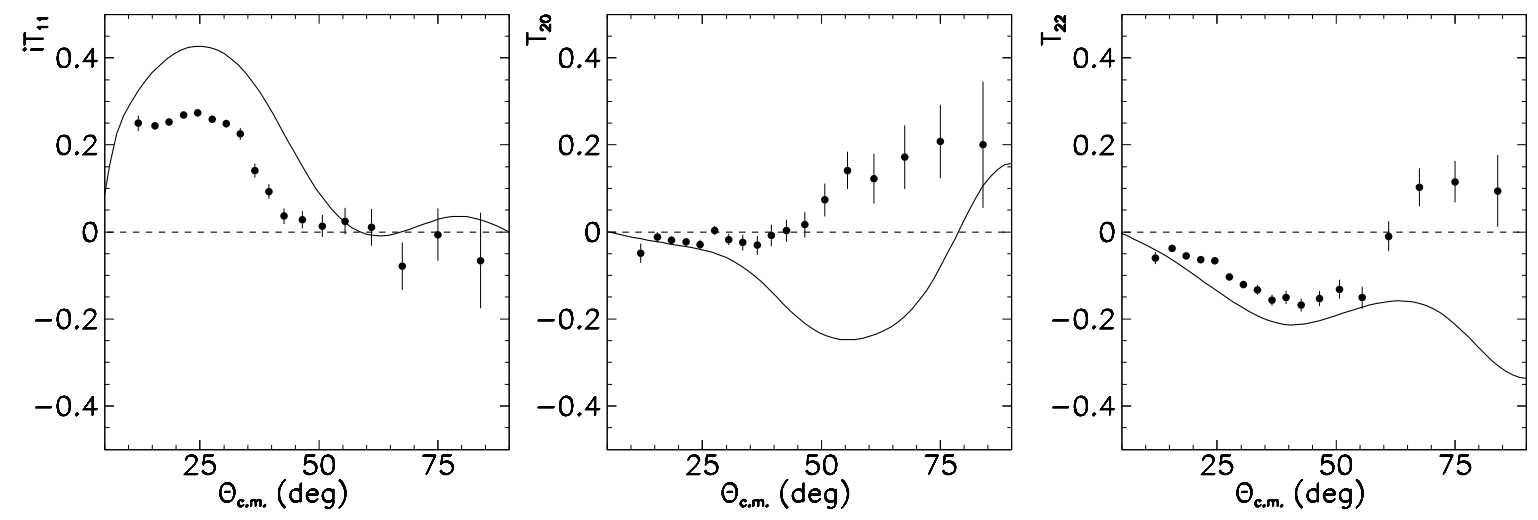

Figure 27. Angular distributions of various analyzing powers for deuteron-deuteron elastic-scattering at an incident beam energy of $231 \mathrm{MeV}$. Errors shown are statistical. The curve shows the result of approximated calculations [316. Reprinted with permission from [316]. Copyright (2007) by the American Physical Society.

the singularity pattern in in the Faddeev equations which might open the way to fournucleon scattering calculations the reader is referred to Ref. [318]. We also emphasize that the four-body continuum dynamics has also been studied using the Lorentz integral transform methof [23].

It is also because of the lack of theoretical predictions that no major attempts have been made in the past to study three-nucleon force effects in the four-body systems at intermediate energies. Nevertheless, recent attempts have been made to contribute to the understanding of nuclear forces in these systems. The experiments are very similar to those in the three-body system except that the number of channels is much higher. All possible channels in the interaction between two deuterons were listed in section 4.2.

The channels with the two-body final state are best studied using a magnetic spectrometer, as a good measure of the scattering angle of one of the outgoing particles is enough to determine the kinematics, provided that the particles are well identified. For the study of the other channels, one has to resort to detection systems such as that shown in Fig. 10 which span a large part of the available phase space of the reaction. Results of a recent measurement at IUCF [316] of elastic deuteron-deuteron scattering is shown in Fig. 27] together with calculations that are the results of very approximate calculations. More recently, two high-precision measurements were performed at KVI aiming to measure all reaction channels mentioned in the previous section. In Fig. 28, the results of vector and tensor analyzing powers for the three-body break-up channel (reaction (iv) in the previous section) are shown for a few selected kinematics [319]. From the figure, it is clear that there is a rich set of data with high precision at this energy which can be used to test the models as they become available at intermediate energies. 


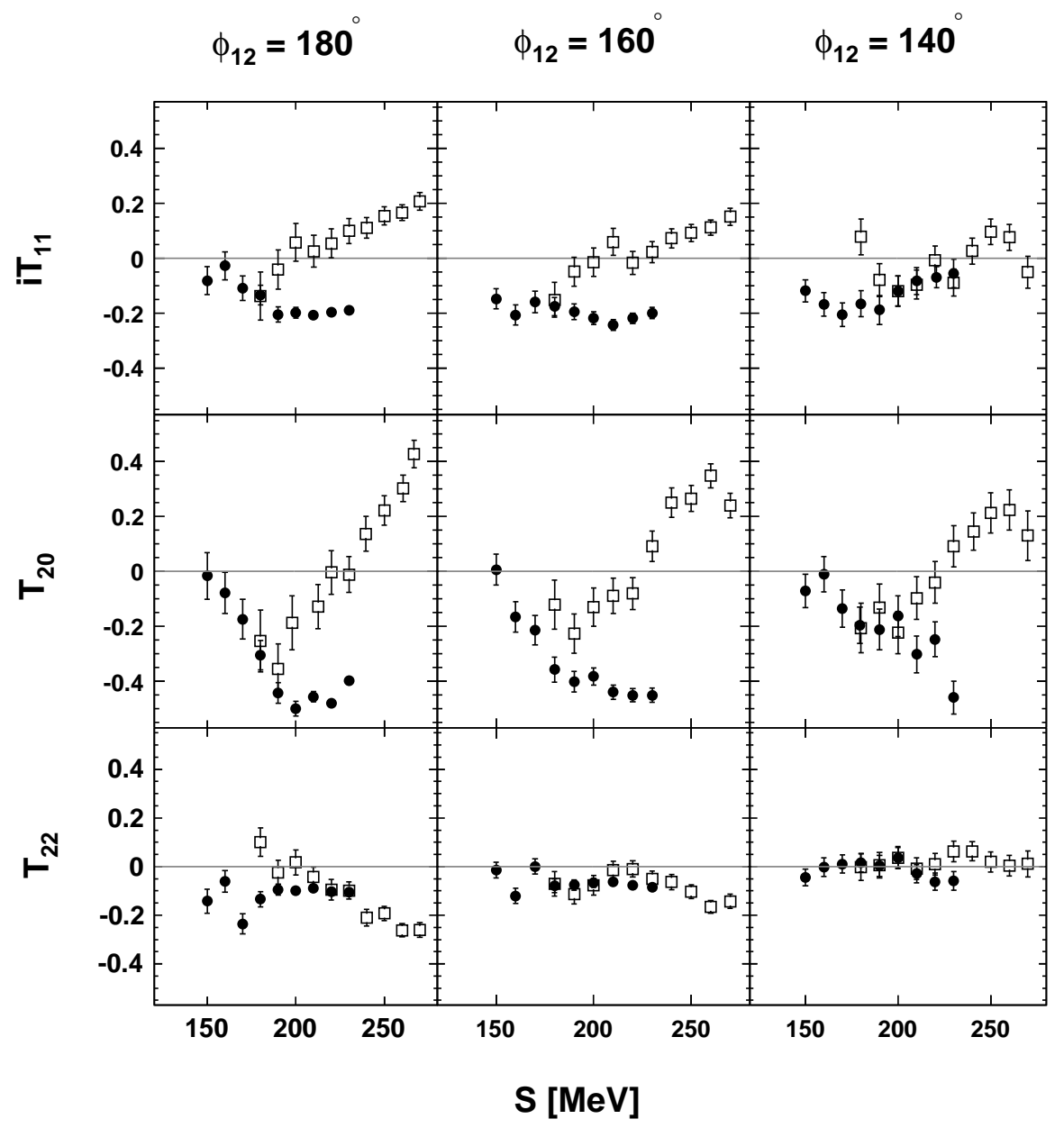

Figure 28. The vector- and tensor-analyzing powers of the reaction $\vec{d}+d \longrightarrow p+n+d$ at $\left(\theta_{d}, \theta_{p}\right)=\left(15^{\circ}, 15^{\circ}\right)$ (open squares) and $\left(\theta_{d}, \theta_{p}\right)=\left(25^{\circ}, 25^{\circ}\right)$ (filled circles) as a function of $S$ for different azimuthal opening angles listed on top of the figure. The data were taken using a polarized deuteron beam with an energy of $135 \mathrm{MeV}$ [319]. The solid lines in all panels show the zero level of the analzying powers. Only statistical uncertainties are indicated. Reprinted with permission from [319]. Copyright (2010) by the American Physical Society.

\section{How do 3NF manifest themselves in $A>4$ systems?}

Whereas the few-body systems are ideal laboratories to study nuclear forces since calculations can be done with high accurcy, the application of the forces to more complex systems is of even more physical interest. Although much progress has been made in recent years, it is still not possible to reliably predict nuclear levels and binding energies for medium mass and heavy nuclei based on NN and 3N interactions. Most approaches are based on effective two-nucleon interactions, which are fitted to a range of nuclear data. Such interactions often fail to predict observables, especially when one explores exotic systems [320]. Assuming the nucleons are the relevant degrees of freedom to describe nuclei, it should be possible to predict such observables based on the underlying nuclear interactions. It is well known that for such studies not only $2 \mathrm{~N}$ forces, but also 
$3 \mathrm{~N}$ forces are quantitatively relevant. In this section, we review approaches to calculate observables in light nuclei based on $2 \mathrm{~N}$ and $3 \mathrm{~N}$ interactions and summarize the quality of the predictions for these systems.

We start with a section on the quality, advantages and disadvantages of several methods and, in the second section, focus on physical issues. We also note that we will not distinguish 3NFs due to truncations of the model space and due to other leftout degrees of freedom. We stress that these contributions cannot be disentangled as becomes clear when we discuss 3NFs in conjuction with the renormalization group. In this context, standard 3NFs are adjusted to absorb the model space (cutoff) dependence of observables. The results are in many ways interesting since they show the significance of $3 \mathrm{NFs}$ and at the same time they point to deficiencies of today's interactions.

\subsection{Computational methods}

Reaching solid conclusions on the structure and significance of various parts of the nuclear Hamiltonian requires computational methods that allow one to reliably predict binding energies, excitation spectra and other properties of light nuclei. Several of such schemes have been developed over years, which provide such solutions for different types of interactions. Many methods imply constraints on the forces or systems to be studied. But they have been benchmarked for observables where several methods can be applied, e.g. for the $A=4$ binding energy in [290]. Therefore, the numerical results are very reliable in their respective range of applicability.

A very broad overview of nuclear binding energies has been obtained using the GFMC method [4]. As can be seen in Fig. 1 in the introduction, the binding energies of the ground state and the low lying excited states have been calculated for many nuclei with $A \leq 12$. The calculation starts from a variational trial wave function. Using an imaginary time propagation, the ground state is projected out of this trial state. For this, a path integral method is applied. Thereby, integrals are calculated with a Monte Carlo method, but sums over quantum numbers are kept explicitly. Therefore, the calculations grow exponentially with $A$ so that the applicability is limited to systems within the $p$ shell. In this way, it is possible to calculate expectation values for many operators. Due to the complexity of the wave functions, it is however not possible to generate them. Usually, the calculation allows one to study the properties of the lowest level for a given set of quantum numbers. It is however also possible, but computationally much more difficult, to obtain excited states for a given quantum number [321].

Results are usually obtained for the class of Argonne interactions since they are given in a suitable local operator form that is required in the implementation of the method. For this class of interactions, however, even exotic, very weakly bound systems like ${ }^{8} \mathrm{He}$ can be studied. Also, the GFMC approach has been generalized to scattering of nucleons on light nuclei [322] enabling the investigation of unbound systems, i.e. ${ }^{5}$ He. These features make GFMC especially interesting to study the charge and isospin dependence of the nuclear force based on the Argonne-Urbana-Illinois set of interactions. 
The no-core shell model (NCSM) is more flexible with respect to the form of the nuclear interaction. Here, the Schrödinger equation for the nucleus is solved in a harmonic oscillator (HO) basis. Thereby, no inert core is assumed. A direct solution for standard interactions would, in general, require a much too large number of $\mathrm{HO}$ basis states and is not possible. Therefore, the problem is first solved for a cluster of two or three nucleons. This solution can be used to define an effective interaction, which is then used in the nuclear structure calculation [291]. Alternatively, a direct solution is possible for very soft interactions obtained in renormalization-group-based approaches to the nuclear interaction. This will be briefly discussed below. At this point, it is possible to calculate and apply the effective interaction up to three-body cluster level [323] taking into account also the 3NFs discussed in the previous sections [153]. The NCSM gives very reliable predictions for the excitation energies of states that are dominated by the lowest HO states. This implies that so-called intruder states such as e.g. the ${ }^{12} \mathrm{C}$ Hoyle state are not well described [324].

In the standard formulation of NCSM, the calculations are feasible for $p$-shell nuclei. For more complex systems, the number of basis states increases dramatically so that converged calculations cannot be obtained anymore. But recently, the approach has been extended to medium mass nuclei [325] based on a restricted NCSM basis. Here not all basis states are taken into account. Instead the importance of each state is a priori estimated perturbatively. In this way, a major fraction of the states can be dismissed before the Schrödinger equation is solved. This so-called importance truncated NCSM has been criticized for its numerical accuracy (see [326, 327]) but promises an interesting possibility for ab-initio calculations of much heavier systems or intruder states.

Another recently developed extension is the combination of the NCSM with the resonating group method (RGM) 328 331. So far this combination has been implemented for nucleon-nucleus and deuteron-nucleus scattering but at this point omitting 3NFs. The scattering problem is solved within a basis of cluster states and relative coordinates of the clusters. The cluster states are obtained from NCSM calculations. It is then possible to implement the antisymmetrizer for states of different clusters (or the spectator nucleon with respect to the nuclear cluster). This enables to calculate a realistic norm kernel for an RGM calculation. The effective RGM interaction can, therefore, be systematically calculated from the underlying nuclear interaction. This approach works nicely for very low energy scattering, where only a few excitations of the clusters are relevant.

For interactions based on EFT, it recently became feasible to solve the problem not based on a nucleonic Hamiltonian but directly on the Lagrangian of the effective field theory [332]. The solution is performed for a discretized space-time lattice. The strength of this approach is its wide range of applicability. Starting from NN systems [333], which enable to determine the low energy constants for the particular regularization implied by the lattice, even light nuclei [332, 334] and neutron matter [335] have been investigated. The lattice calculations for nuclear structure result in binding energies accompanied with reliable error bounds. The uncertainty estimates do not only take 
into account the numerical errors due to statistical fluctuations of the applied Monte Carlo scheme and lattice artifacts but also the uncertainties of the nuclear Lagrangian by considering several orders of the chiral expansion. In this approach, as is also true for GFMC, nuclear wave functions cannot be computed. Instead, expectation values of operators, e.g. correlation functions, can be computed. Recently, even excited states for ${ }^{12} \mathrm{C}$ have been extracted including the weakly bound Hoyle state that is relevant for nucleosynthesis [336]. Since NN and 3NFs are automatically included when the pertinent terms of the Lagrangian are taken into account, this calculation is in fact the first calculation of this state based only on a few-nucleon input.

For low-momentum interactions, many-body perturbation theory (MBPT) is another very flexible approach to the nuclear many-body problem. It has been applied to finite nuclei [337, 338] as well as to nuclear matter [339 341]. For a review, we refer to Ref. [342]. In this scheme, the solution is based on the Hartree-Fock approximation to the observables, which is particularly simple for closed-shell nuclei and for nuclear matter. For low momentum interactions, this first-order approximation is already sufficiently close to the solution, so that a perturbative expansion for the higher-order contributions becomes meaningful. It has been shown that second-order contributions are significant. Higher-order contributions have only partly been taken into account, but the results indicated that they are negligible. So far only the nuclear matter calculations involve 3NFs. These can be exactly taken into account for the leading, Hartree-Fock, term. For higher orders in the MBPT expansion, state-of-the-art calculations require approximations of the $3 \mathrm{NF}$. Since these higher-order contributions are generally small, it is expected that the approximations only lead to insignificant errors of the calculation.

Also the coupled-cluster approach [343], which is especially suitable for medium mass closed-shell nuclei, has been applied to Hamiltonians that involve 3NFs [344 346]. Early calculations were performed approximating the 3NF by an effective NN force [344, 345]. Based on such calculations, it has already been argued that the residual 3NFs are indeed small [347]. So far, the calculations involving the complete 3NFs 346] have only been performed for ${ }^{4} \mathrm{He}$ since the computational demands are very high. It turned out, for this system and a specific choice of NN interaction, that the proper 3NF contributions are tiny. The bulk of the $3 \mathrm{NF}$ effects could be absorbed in effective NN forces, see also Ref. [345] for a related earlier study. At this point, for more complex systems, the importance of 3NFs is often estimated based on variations of the NN force (e.g. via a cutoff of a low-momentum interaction). In some case, this indicates that a complete treatment of 3NFs might be required in such systems [348].

This concludes our survey on methods that have been applied to the nuclear manybody problem based on $\mathrm{NN}$ and $3 \mathrm{~N}$ interactions. We would like to mention briefly that other techniques have been developed that promise the capability to employ NN and $3 \mathrm{~N}$ forces to systems with $A>4$, i.e. the Fermion Molecular Dynamics (FMD) 349] and the effective interaction hyperspherical harmonics approach (EIHH) [350]. FMD has been used with modified $\mathrm{NN}$ interactions for a wide variety of nuclear systems including the Hoyle state [351] and has proven to provide a flexible ansatz for the 
nuclear wave function that can accommodate shell-model-like states and cluster-like states simultaneously. Unfortunately, 3NFs have not been incorporated yet. EIHH has already been used for $A=4$ including 3NFs [352,353], but, for $A>4$, it has only been applied using simplified NN interactions [354].

\subsection{Signatures of $3 N F$ s for $A>4$}

Based only on the phenomenological NN interactions, the binding energies of light nuclei are underpredicted [4, 355] as can be expected from the results for $A \leq 4$ systems. This underbinding is indeed much reduced, once $3 \mathrm{NF}$ s are taken into account that are adjusted to reproduce the binding energies of light systems [4,153]. The comparably small deviations from the experimental binding energies observed for the combined interactions is nevertheless a first interesting hint that $p$-shell nuclei are much more sensitive to details of the $3 \mathrm{NF}$ s than the ${ }^{4} \mathrm{He}$ nucleus.

First of all, one observes that the binding energies predicted based on AV18 (or a simplified version AV8' [356]) in combination with the Urbana-IX or TM 3NFs are smaller than obtained from experiment. The deviations are small compared to the overall binding energy and on the percent level, when compared to the potential energy. Nevertheless the deviations can be significant in many cases since the ordering of levels is affected. A prominent example is the ordering of the $J^{\pi}=3^{+}$and $J^{\pi}=1^{+}$levels in ${ }^{10} \mathrm{~B}$. Experimentally, the splitting of both states is $720 \mathrm{keV}$ where $J^{\pi}=3^{+}$is the ground state of ${ }^{10} \mathrm{~B}$. Clearly, the level splittings of $p$-shell nuclei are tiny compared to binding energies/potential energies. Therefore, it is not surprising that AV18 alone and in combination with Urbana-IX leads to a $J^{\pi}=1^{+}$ground state. This remains true if other phenomenological $\mathrm{NN}$ interactions are used [355]. Interestingly, the addition of the TM 3NFs at least results in the correct ordering of levels although the splitting seems to be smaller than experiment [153]. These results make two facts obvious: the binding energies and splittings of levels of $p$-shell nuclei are extremely sensitive to details of the nuclear Hamiltonian and rather small deviations can lead to qualitatively different results since the ordering of levels can be affected with the obvious impact on possible transitions between the levels. At the same time, one can expect an especially large sensitivity to $3 \mathrm{NFs}$ that one only finds in very specific kinematical conditions in $3 \mathrm{~N}$ and $4 \mathrm{~N}$ scattering, e.g. in cross section minima. The systematic studies discussed in the previous chapters are, therefore, not only academic exercises but are required to determine nuclear Hamiltonians that are accurate enough for systematic predictions of nuclear structure.

The GFMC studies revealed another interesting failure of the combination of AV18 and Urbana-XI. The deviations of predictions from the experimental results increased with an increasing number of neutrons [154]. This clearly points to a deficiency in the isospin dependence of this combination of nuclear interactions. This observation triggered the development of new 3NFs models. The GFMC collaboration identified socalled ring diagrams, $3 \pi$-exchanges with intermediate $\Delta$ excitations, as a possible source 
of additional isospin $T=3 / 2$ contributions of the 3 NFs. They added the expected new spin/isospin structures to their model which culminated the series of Illinois interactions (IL1 to IL5) [154]. Indeed, their study showed that the new interaction terms further improved the description of the spectra of $p$-shell nuclei. The new terms resolved the underbinding problem for neutron-rich systems and the deviations in the level orderings at the same time [357. These improvements could also be identified for $n-{ }^{4} \mathrm{He}$ scattering [322]. Note that these original calculations suffered from a bug in the threenucleon force code [5] which is resolved in the meantime, and lead to the development of a new parameter set (IL7) of the Illinois 3NF. Figure 1 summarizes the corrected results which qualitatively agree with the old ones.

A close look reveals that the $3 \mathrm{NF}$ contributions due to triples of neutrons are very small for $p$-shell nuclei probably because the additional neutrons are usually well separated from each other and from the core of the neutron-rich nucleus [358]. This is very different for neutron matter and it turned out that the Illinois model, that describes $p$-shell nuclei very well, is not suitable for neutron matter [155]. An improved version is under development [5].

Although the results for TM' are in some aspects different to the ones of UrbanaIX and/or Illinois, the calculations always showed that the overall agreement with experiment is clearly improved by the addition of 3NFs. Looking more into details, the results still show deviations from data similarly to calculations based on Urbana-IX. This situation can already be expected from the fact that also the $p d$ and $n d$ scattering data are not satisfactorily described.

Since today's combinations of NN interactions and 3NFs do anyway not describe nuclei perfectly and because of the technical complexity to apply $3 \mathrm{NFs}$ in nuclear structure calculations, there have been attempts to get around 3NFs by modifying NN interactions. Most prominently the INOY (inside non-local outside Yukawa) model [359] and the JISP (J-matrix inverse scattering potentials) [360] are phase-shift equivalent realistic NN interaction that are engineered to improve the description of binding energies. The INOY model reproduces the ${ }^{3} \mathrm{H}$ binding energy exactly. But calculations for $p$-shell nuclei showed discrepancies to charge radii, spectra and binding energies again [361]. One is lead to conclude that also this model requires the addition of $3 \mathrm{NFs}$ although $s$-shell systems might be described without them. Interestingly, a modified version of INOY, where $\mathrm{P}$-wave NN phase shifts have been altered so that the $n d A_{y}$ is properly described, leads to a good description of some spin-orbit (LS) splittings in $p$ shell nuclei. This makes a relation of both discrepancies likely. As the name suggest, the long-range part of the interaction nevertheless is driven by the $1 \pi$-exchange so that it is conceivable that INOY results can be improved by adding 3 NFs based on $2 \pi$ exchange to improve this situation. JISP, in contrast, is not only fitted to NN data. Using a scheme of unitary transformations, JISP could be fitted to $p$-shell spectra while keeping the description of the NN data. Therefore, both, $p$-shell spectra and NN data are described well. Despite this success, it remains to be seen to what extent predictions are possible in more complex systems. Since the unitary transformations also change the long-range 
part of the interaction, it is also not clear how JISP could be improved by 3NFs, if discrepancies to data are found.

The huge number of possible operator structures, estimated to be at least 120, makes a purely phenomenological approach to the 3NFs impossible. Clearly, it would be helpful to improve the 3NFs according to some theoretical guidance and chiral EFT promises such a guidance. As discussed before, the leading $3 \mathrm{NF}$ in chiral EFT is given by the usual $2 \pi$-exchange and additional $1 \pi$ and contact interactions (see Fig. 17). Therefore, using the NCSM, the impact of these additional structures on observables involving $p$ shell nuclei has been studied. In a first step, ${ }^{7} \mathrm{Li}$ was investigated [295]. In this first study, the spectrum of ${ }^{7} \mathrm{Li}$ was compared based on chiral interactions. The parameters of the chiral 3NFs were chosen to be consistent with the NN interaction used and the two parameters related to the $1 \pi$ and contact terms used to fix the binding energies of ${ }^{3} \mathrm{H}$ and ${ }^{4} \mathrm{He}$ exactly at their experimental values. As expected, the results for ${ }^{7} \mathrm{Li}$ differed for both choices of these parameters showing that the additional shorter-range contributions are significant for $p$-shell nuclei.

It was, however, not clear which choice of parameter set resulted in a better description. More research on the impact of the shorter-range contributions revealed that the fitting procedure of [295] missed that also the density of ${ }^{4} \mathrm{He}$ considerably depended on the parameters of the 3NF. Given that the binding energy of ${ }^{4} \mathrm{He}$ might get contributions from several subleading few-nucleon interactions including the $4 \mathrm{NF}$, it became clear that fitting the parameters to the density (or matter radius) of ${ }^{4} \mathrm{He}$ and the ${ }^{3} \mathrm{H}$ binding energy is a more reliable approach. This was first done in [284]. This work studied a wide range of observables for several $p$-shell nuclei. Using the density of ${ }^{4} \mathrm{He}$, a preferred set of strength constants for the leading $3 \mathrm{NF}$ was found. Then the combinations of the strength were modified keeping the ${ }^{3} \mathrm{H}$ binding energy constant and allowing for slight variations of the predictions for the density. In this way, it was found that most excitation energies and transition matrix elements were only mildly dependent on the choice of strength constants.

A few of the observables turned out to be very sensitive to the contribution of the short range 3NFs: e.g. the quadrupole moment of ${ }^{6} \mathrm{Li}$, the ratio of the electric quadrupole strength $B(E 2)$ for transitions of the ground state of ${ }^{10} \mathrm{~B}$ to the first and second excited $J^{\pi}=1^{+}$states, and the magnetic dipole strength $B(M 1)$ for transitions from the ground state of ${ }^{12} \mathrm{C}$ to the $J^{\pi}=1^{+}$isospin 0 state. Within the accuracy of the calculations, it was possible to find a choice for the strength of the short-range contributions which consistently describes all these observables and the density of ${ }^{4} \mathrm{He}$. The spectra of the considered $p$-shell nuclei generally improved by adding the chiral 3NF in this form. Still, a few discrepancies hint to missing contributions or inaccurately determined parameters of the interactions. Again, this is in line with the situation in few-nucleon systems, where a few observables are still not well described. Since many of the discrepancies are related to LS splittings, it is conceivable that such small deviations might be related to the $A_{y}$ problem. It will be interesting to study the effect of subleading $3 \mathrm{NFs}$ on such observables and a possible correlation with $A_{y}$. 
The nuclear lattice simulations carried out so far out so far are in line with the results that the properties of nuclei are qualitatively described at $\mathrm{N}^{2} \mathrm{LO}$. Here, the convergence of the ground and excited states with respect to the order of the chiral expansion was studied in more detail. Looking at predictions at NLO and $\mathrm{N}^{2} \mathrm{LO}$, it became clear that a quantitative description requires $\mathrm{N}^{3} \mathrm{LO}$ contributions which have not been completely included yet in any of the approaches [336].

A large fraction of studies involving $3 \mathrm{NFs}$ is based on renormalization group ( $\mathrm{RG}$ ) approaches to the nuclear force. The $V_{\text {low }-k}$ [362 364], the similarity renormalization group (SRG) [365] and the unitary correlation operator method (UCOM) [366] belong to this category. By different means one of the phenomenological NN interactions is softened such that the long range part of the interactions is unaltered. The procedures guarantee that NN phase shifts do not change for momenta below a cutoff momentum. Such a softening is a pre-requisite to solve the nuclear many-body problem based on $\mathrm{NN}$ and $3 \mathrm{~N}$ interactions. For $V_{l o w-k}$ and SRG it was explicitly shown that the softened NN interaction are universal meaning that they do not depend on the NN interaction from which one starts the procedure. The softening implies a dependence of the results on the RG parameter (or cutoff) which was shown to be of the size expected for 3NFs and should be mainly absorbed by 3NFs [367]. The similarity of the interactions to chiral interactions in some cutoff range makes it conceivable that RG interactions may be consistently augmented by chiral few-nucleon forces. The application of such combinations to nuclear structure revealed that, for a limited cutoff range, the NN interaction and the $3 \mathrm{~N}$ interaction can be perturbatively added to a Hartree-Fock solution [339, 341] (for a review see [368]). The results show that the $3 \mathrm{NF}$ contribution is of the size expected. But this implies that it is necessary to obtain a realistic saturation point for nuclear matter. The cutoff dependence of the results is very much reduced by the $3 \mathrm{NFs}$ indicating that $4 \mathrm{~N}$ and higher-order interactions are still less important. The calculations for nuclear matter also showed a large sensitivity of the results on the strength parameter of the $2 \pi$-exchange part of the chiral $3 \mathrm{NF}$ [340, 341. Since the uncertainties of these parameters are rather large, a possible determination from few-nucleon data, as started in [167], is certainly well motivated. Such determinations should, however, carefully address the theoretical uncertainty resulting from the truncation of the chiral expansion for the nuclear forces.

Similar calculations for UCOM for finite nuclei were performed without $3 \mathrm{NF}$ and, for an optimized correlation parameter, showed a rather good description of binding energies [337]. But it turned also out that radii are not well reproduced and again the need for 3NFs was confirmed. Very recently short-ranged 3NFs could be included in similar calculations [369] improving the agreement with data.

The RG approaches have the important advantage that a cutoff parameter can be varied in certain limits similarly to the chiral EFT approach. The cutoff dependence of results is then a lower bound of the accuracy achieved in calculations and gives a first estimate of omitted contributions, e.g. higher-order few-nucleon interactions. It can also be used to identify correlations between observables as the extensions of the Tjon 
line to more complex systems [370]. For SRG, this has even been extended to generate some parts of the missing 3NFs [371]. Such developments enable one to give better estimates for, e.g., the $4 \mathrm{NF}$ contribution (which was shown to be small in this case) but they also allow to determine the $3 \mathrm{NF}$ for arbitrary cutoffs starting from a single fit to data 371.

A further interesting application of 3NFs is reported in 372. Within the standard shell model, the oxygen isotopes are studied in more detail. It is shown that calculations based on phenomenological NN interactions fail to reproduce the position of the dripline correctly since they results in too low single energies for the neutron $d_{3 / 2}$ orbitals. $3 \mathrm{NFs}$ correct this failure leading to the correct position of the drip-line at ${ }^{24} \mathrm{O}$.

This short overview concentrated on the results for binding energies. Indeed, these are especially sensitive to $3 \mathrm{NFs}$, but we stress that many nuclear structure calculations showed that 3NFs also have impact on transition matrix elements. In some cases, transition matrix elements are strongly dependent on a suitable interplay of wave function components. This has been exemplified e.g. in [373 375] for neutrino scattering and the lifetime of ${ }^{14} \mathrm{C}$, see also Ref. [376] for a related recent work.

In summary, the 3NFs have been shown to be important ingredients for nuclear structure calculations. Although, for the $p$-shell, the predictions are already in fair agreement with the data, there are still discrepancies. It is hoped that such discrepancies can be resolved by higher-order EFT interactions. It will be interesting to observe how nuclear structure and few-body results are affected by the next generation of $3 \mathrm{~N}$ and higher-order interactions. At the same time, the development of RG methods to make the nuclear many-body problem tractable highlighted the importance of 3NFs to remove the scale dependences of these approaches when only NN interactions are used.

\section{Summary, conclusions and outlook}

In this review we have summarized recent theoretical and experimental achievements towards understanding the role of the three-nucleon force in few-nucleon systems and its application in many-nucleon systems. Resolving the structure of the three-nucleon force requires accurate experimental data and the ability to accurately solve the few-body Schrödinger equation for a given nuclear Hamilton operator. Considerable progress has been made in the past decade on both fronts.

Clearly, the most natural place to test the $3 \mathrm{NF}$ is the three-nucleon continuum. Neutron-deuteron scattering calculations can nowadays be routinely performed with and without 3NFs both for the elastic and the break-up channels. Since most of the data are proton-deuteron data, one needs to take into account the long-range electromagnetic interaction in the Faddeev equations which is especially important in various proton-deuteron break-up configurations. Recently, impressive progress towards resolving this long-standing challenge was made albeit the final word is perhaps still to come. The experimental techniques have improved drastically in the course of time and were partly driven by the precision requirements in the field of few-nucleon systems. 
The experimental situation in $\mathrm{N} d$ scattering at intermediate energies is summarized in Fig. 12, It clearly demonstrates that the existing database is far from being complete. Most of the data corresponds to differential cross sections and nucleon and deuteron analyzing powers. A restricted number of more complicated spin observables such as the spin transfer coefficients have also been measured. The global impact of the phenomenological 3NF models on the available data is visualized in Figs. 20, 21 and 22 for elastic scattering and Figs. 23 and 24f for the break-up observables. The experimental data are confronted with the theory calculations by the Hanover-Lisbon group based on the coupled-channel version of the CD-Bonn potential which allows one to identify the effects of the $3 \mathrm{NF}$ due to intermediate $\Delta$ excitations. This particular choice can serve as a representative example of three-nucleon calculations based on phenomenological high-precision potentials accompanied by 3NF models, see Fig. 20, The elastic cross section and the nucleon vector analyzing power show a clear signature of the $3 \mathrm{NF}$ with the deviations from the data being significantly reduced once the $3 \mathrm{NF}$ is included. On the other hand, sizable deviations, typically increasing with energy, still persist after inclusion of the 3NF especially at backward angles indicating deficiencies in the 3NF models. It is difficult to make a definite global conclusion for tensor analyzing powers and spin-transfer/correlation coefficients where the situation is more controversial (which is, in part, due to the less precise and much smaller amount of data available). The analysis of the break-up data shows that the effects of the 3NF are predicted to be, in most cases, fairly small and insufficient to remove the discrepancies with the theory. This is especially true for the vector analyzing power indicating the possible deficiencies in the spin structure of the current 3NF models.

Given the recent progress towards rigorous solution of the four-body problem in the continuum which is, however, presently limited to the low-energy region, four-nucleon systems are expected to be a promising testing ground for three- and four-nucleon forces in the near future. It should also be emphasized that the four-nucleon systems feature certain properties, such as the low-lying resonances, which are absent in $3 \mathrm{~N}$ scattering and are expected to increase the sensitivity to the details of the nuclear Hamiltonian. One well-established puzzle in the $4 \mathrm{~N}$ continuum is related to the total cross section for neutron-triton scattering showing a broad peak around $E_{\text {lab }} \sim 3 \mathrm{MeV}$ due to several $\mathrm{P}$-wave resonances, which appears to be underpredicted by the existing two- and threenucleon force models. The $A_{y}$ puzzle also persists in the $4 \mathrm{~N}$ system although a recent calculation by the Pisa group indicates a significant improvement due to inclusion of the chiral $3 \mathrm{NF}$ at $\mathrm{N}^{2} \mathrm{LO}$. Data at intermediate energy are rather scarce for this system. The lack of data for this system is primarily due to the lack of exact calculations. Only recently, precision data for various reaction channels and covering a large part of the phase space have become available. The four-nucleon continuum will clearly be an important frontier area of research in the next years.

Spectra of light nuclei provide another interesting testing ground for three-nucleon forces. Calculations performed within the GFMC and NCSM approaches typically show a clear improvement once the $3 \mathrm{NFs}$ (chiral $3 \mathrm{NF}$ at $\mathrm{N}^{2} \mathrm{LO}$, Urbana IX, Illinois $3 \mathrm{NF}$ 
models and TM') are included. Sizable deviations, however, still persist pointing to the deficiencies in the spin- and isospin structure of these $3 \mathrm{NF}$ models.

The results of the present analysis demonstrate clearly that the momentum-spinisospin structure of the $3 \mathrm{NF}$ is not properly described by the existing phenomenological models. What is needed is a systematically improvable theoretical framework which allows to derive consistent two- and many-nucleon interactions and currents. Such a framework is provided by chiral effective field theory. Two- and many-nucleon forces in this approach are derived from the underlying effective Lagrangian and do not suffer from conceptual problems typically arising in the context of phenomenological models (such as off-shell effects). In the past decade, $\mathrm{NN}$ potentials at $\mathrm{N}^{3} \mathrm{LO}$ in the chiral expansion have been developed and demonstrated to allow for an accurate description of the two-nucleon data comparable to the one of the high-precision potentials. The three-nucleon force has so far only been explored at order $\mathrm{N}^{2} \mathrm{LO}$ where it first starts contributing to the nuclear Hamiltonian. Its short-range part depends on the two new LECs $c_{D}$ and $c_{E}$ which cannot be determined in the $2 \mathrm{~N}$ system. The $c_{D}$-term is governed by the $\mathrm{NN} \rightarrow \mathrm{NN} \pi$ transition which figures importantly in strong, electromagnetic and weak few-nucleon reactions. The exciting possibility to bridge these very different processes is already being explored by several groups, but more quantitative studies should be carried out in the future. The description of the low-energy neutron-deuteron scattering observables at $\mathrm{N}^{2} \mathrm{LO}$ is, in general, comparable to the one obtained from the high-precision potential models showing similar puzzles such as the cross section in the space-star break-up configuration. While the results at intermediate energies are generally in a good agreement with the data, the theoretical uncertainty becomes rather large reflecting similar pattern in the $2 \mathrm{~N}$ sector at this order in the chiral expansion. Promising results were obtained in calculations based on the $\mathrm{N}^{3} \mathrm{LO} \mathrm{NN}$ potentials accompanied with the $\mathrm{N}^{2} \mathrm{LO} 3 \mathrm{NF}$. In particular, the inclusion of the $3 \mathrm{NF}$ was shown to improve description of the spectra of light nuclei and to reduce the $A_{y}$ puzzle in the $4 \mathrm{~N}$ system. Another interesting abinitio approach to few- and many-nucleon systems combines effective field theory with numerical lattice simulations. Recently, this method has been successfully applied at $\mathrm{N}^{2} \mathrm{LO}$ to compute the ground state energies of ${ }^{4} \mathrm{He},{ }^{8} \mathrm{Be}$ and ${ }^{12} \mathrm{C}$ and the Hoyle state which presents a major challenge for nuclear theory.

The first corrections to the $3 \mathrm{NF}$ at $\mathrm{N}^{3} \mathrm{LO}$ are becoming available and currently being implemented in $3 \mathrm{~N}$ scattering calculations. It is worth mentioning that they do not involve any additional free parameters. While definite conclusions about the importance of subleading corrections to the $3 \mathrm{NF}$ can only be made after performing explicit calculations, the preliminary estimations of the longest-range contributions indicate that the effects in nucleon-deuteron scattering might be fairly small [168]. If so, it would be necessary to extend the calculations to an even higher order in the chiral expansion to resolve the puzzles in the $3 \mathrm{~N}$ continuum. A more promising approach to improve the convergence of the chiral expansion is to explicitly include the spin- $3 / 2$ degrees of freedom corresponding to the $\Delta(1232)$ isobar which is well known to play an important role in nuclear dynamics due to its low excitation energy and strong coupling 
to the $\pi N$ system. Such explicit treatment of the $\Delta$ in the EFT leads to more natural values of the LECs and allows one to resum a certain class of important contributions. The improved convergence of the resulting approach in the NN sector was recently confirmed in peripheral nucleon-nucleon scattering at $\mathrm{N}^{2} \mathrm{LO}$. The implications for $3 \mathrm{~N}$ scattering are yet to be worked out. While the complete expressions for the $3 \mathrm{NF}$ at $\mathrm{N}^{2} \mathrm{LO}$ are exactly the same in both approaches (althouth certain contributions in the $\Delta$-full theory are shifted from $\mathrm{N}^{2} \mathrm{LO}$ to $\mathrm{NLO}$ ), one expects sizable long-range $\mathrm{N}^{3} \mathrm{LO}$ contributions to the $3 \mathrm{NF}$ and $4 \mathrm{NF}$ due to intermediate $\Delta$-excitations, see also [377] for a related discussion. The calculations by the Argonne group using the phenomenological Illinois $3 \mathrm{NF}$ models motivated by the ring diagrams with an intermediate $\Delta$-excitation seem to indicate the importance of such contributions. It is worth mentioning that $3 \mathrm{NFs}$ due to two and three intermediate $\Delta$-excitations neglected by the Argonne and Hanover-Lisbon groups also appear at $\mathrm{N}^{3} \mathrm{LO}$, and the resulting potentials turn out to be of a similar size. Last but not least, the four-nucleon force also starts to contribute at $\mathrm{N}^{3} \mathrm{LO}$. The parameter-free expressions have recently been worked out in the $\Delta$-less formulation and shown to yield a small contribution to the $\alpha$-particle binding energy of the order of a few hundred $\mathrm{keV}$. It remains to be seen whether this conclusion will still hold after explicit inclusion of the $\Delta$-isobar although the results in the $4 \mathrm{~N}$ system obtained by the Hanover-Lisbon group seem to indicate a small contribution.

The question to be raised is whether one should do more measurements and if so, what should they be? As shown in Fig. 12, cross sections and (proton and deuteron) analyzing powers have been measured at several energies and for a large range of scattering angles, and aside from some normalization problems, the database is in good shape for these two observables. The situation is, however, different for more exclusive spin observables such as spin-transfer and spin-correlation coefficients which have been measured only for a couple of energies and in a limited angular coverage. Different combinations of spins in these observables clearly show different behavior making them suitable for these studies.

In the break-up sector, there is certainly room for improvement for all observables. However, due to the difficulties in performing the experiments, the measurements should be guided by theoretical input as to where the effects would be largest. Also, for this reaction channel, more exclusive spin observables should be measured in order to fully understand the underlying dynamics. It should, however be mentioned that observables like spin-transfer coefficients are the most difficult to measure for a large part of the phase space as one would require a polarimeter in several regions of the phase space. First attempts have been made to measure this observable for a selected kinematics [264]. The only laboratories which are, in principle, capable of carrying out this type of measurements at intermediate energies are RIKEN, RCNP and Jülich.

The situation for four-nucleon systems is similar to that of the three-nucleon system of about 20 years ago. Measurements exist only to a limited extent and primarily at energies below the break-up threshold. Note that the large efforts undertaken in the three-nucleon sector in the last two decades were due to the availability of exact 
calculations justifying large investments for these measurements. In that respect, the theoretical efforts in the four-nucleon sector are clearly lagging behind and should be vigorously pursued to prove the feasibility of doing exact calculations at intermediate energies before major efforts are put into the actual measurements of observables.

In short, a lot has been learned about nuclear forces and few-nucleon systems in the past two decades making this field of science almost an exact field where the conclusions are drawn based on precise, quantitative arugments. Accurate calculations can be performed routinely in two- and three-body systems using various potential models. The results of these calculations can be examined with the precise data which are available for these systems. All observables can be investigated and remarkable agreements have been reported in a large part of the phase space for all possible reactions. However, there are also clear discrepancies which are much larger than any experimental or theoretical uncertainties pointing to the fact that the nuclear Hamiltonian still needs refinement. It has been shown that the disagreements in cross sections and analyzing power for the elastic channel increase with incident energy. More exclusive spin observables show discrepancies to various degrees depending on the combination of the spin components. In selected break-up kinematics, S-wave proton-proton pairs were observed at small relative energies and sizable discrepancies were observed with theory predictions for the vector analyzing power, $A_{y}$. Strikingly, data taken at similar elastic kinematics in which the deuteron was detected, were described significantly better by the same theory. All these observations call for a systematic and quantitative theoretical approach for the nuclear forces and few-nucleon dynamics. To establish such an approach in the complete energy range up to the pion-production threshold still poses a challenge for a theory. The ongoing efforts in the chiral effective field theory should be further pursued to extend $3 \mathrm{~N}$ calculations which already exist to higher energies and to reduce the theoretical uncertainty. Based on the experience from the $2 \mathrm{~N}$ system, this will require to go to at least $\mathrm{N}^{3} \mathrm{LO}$ in the chiral expansion of the $3 \mathrm{NF}$ and, possibly, to include the $\Delta$ isobar as an explicit degree of freedom. Work along these lines is in progress, and will certainly stimulate dedicated experiments in $\mathrm{N} d$ scattering to explore and test the spin structure of the emerging, novel 3NFs. In parallel, efforts should be increased towards accurate calculations of four-nucleon scattering observables as they promise to be a good testing ground to study nuclear forces. Once these systems are under control, steps can be taken to understand the full dynamics of nuclear systems with more nucleons involved. On the computational front, various groups have developed techniques to calculate complicated many-body systems including 3NFs. Some of these new developments rely on RG evolved nuclear interactions, where the $3 \mathrm{NF}$ is an essential ingredient to remove the scale dependence of results. Independent of this issue, the input from few-nucleon systems will clearly be crucial to eventually understand the more complex systems. Nuclear structure and reaction studies will undoubtedly benefit from the present developments. 
Signatures of three-nucleon interactions in few-nucleon systems

\section{ACKNOWLEDGEMENTS}

Writing a review article entails discussing various aspects with many people. The input of so many colleagues has been essential in bringing this article to a successful end. Here, we mention the names of all colleagues who have given very valuable comments on various parts of the article alphabetically and would like to express our gratitude toward them. They are: S. Coon, A. Deltuva, A.C. Fonseca, R.J. Furnstahl, W. Glöckle, J. Golak, F. Gross, H.-W. Hammer, M.N. Harakeh, H. Kamada, A. Kievsky, St. Kistryn, U. van Kolck, D. Lee, R. Machleidt, J.L. Matthews, U.-G. Meißner, H.O. Meyer, P. Navratil, G. Orlandini, T. Papenbrock, S. Pieper, K. Sagara, H. Sakai, P.U. Sauer, H. Paetz gen. Schieck, A. Schwenk, K. Sekiguchi, E. Stephan, E. Stephenson, P. Thörngren Engblom, R.G.E. Timmermans, W. Tornow, M. Viviani, and H. Witała. Further, we would like to thank all our collaborators for having helped us in all aspects of our work which led to this review article. Part of the numerical calculations were performed at the JSC, Jülich, Germany. N. KalantarNayestanaki and J.G. Messchendorp are financially supported by the University of Groningen $(\mathrm{RuG})$ and the Helmholtzzentrum für Schwerionenforschung GmbH (GSI), Darmstadt. E. Epelbaum would like to acknowledge the financial support of the Helmholtz Association (contract number VH-NG-222) and the European Research Council (ERC-2010-StG 259218 NuclearEFT).

\section{References}

[1] A. Nogga, H. Kamada, and W. Glöckle, Phys. Rev. Lett. 85, 944 (2000), arXiv:nucl-th/0004023.

[2] A. Nogga et al., Phys. Rev. C 67, 034004 (2003), arXiv:nucl-th/0202037.

[3] P. Doleschall, I. Borbely, Z. Papp, and W. Plessas, Phys. Rev. C 67, 064005 (2003).

[4] S. C. Pieper and R. B. Wiringa, Ann. Rev. Nucl. Part. Sci. 51, 53 (2001), arXiv:nucl-th/0103005.

[5] S. C. Pieper, private communication.

[6] W. Glöckle, H. Witała, D. Hüber, H. Kamada, and J. Golak, Phys. Rept. 274, 107 (1996).

[7] L. E. Marcucci, A. Kievsky, L. Girlanda, S. Rosati, and M. Viviani, Phys. Rev. C 80, 034003 (2009), arXiv:0905.3306[nucl-th].

[8] K. Sagara et al., Phys. Rev. C 50, 576 (1994).

[9] K. Sagara, Few Body Syst. 48, 59 (2010), 10.1007/s00601-010-0096-6.

[10] K. Sagara, private communication.

[11] M. H. Wood et al., Phys. Rev. C 65, 034002 (2002), arXiv:nucl-ex/0110014.

[12] S. Shimizu et al., Phys. Rev. C 52, 1193 (1995).

[13] J. Ley et al., Phys. Rev. C 73, 064001 (2006).

[14] P. F. Bedaque and U. van Kolck, Ann. Rev. Nucl. Part. Sci. 52, 339 (2002), arXiv:nuclth/0203055.

[15] S. R. Beane, P. F. Bedaque, W. C. Haxton, D. R. Phillips, and M. J. Savage, (2000), arXiv:nuclth/0008064.

[16] E. Epelbaum, Prog. Part. Nucl. Phys. 57, 654 (2006), arXiv:nucl-th/0509032.

[17] E. Epelbaum, H.-W. Hammer, and U.-G. Meißner, Rev. Mod. Phys. 81, 1773 (2009), arXiv:0811.1338[nucl-th].

[18] H. Arenhovel et al., (2004), arXiv:nucl-th/0412039.

[19] J. Purcell, J. Kelley, E. Kwan, C. Sheu, and H. Weller, Nucl. Phys. A848, 1 (2010).

[20] H. W. Hammer and L. Platter, Ann. Rev. Nucl. Part. Sci. 60, 207 (2010), arXiv:1001.1981[nucl- 
th].

[21] J. Golak et al., Phys. Rept. 415, 89 (2005), arXiv:nucl-th/0505072.

[22] J. Carlson and R. Schiavilla, Rev. Mod. Phys. 70, 743 (1998).

[23] S. Bacca, N. Barnea, W. Leidemann, and G. Orlandini, Phys. Rev. Lett. 102, 162501 (2009), arXiv:0811.4624[nucl-th].

[24] S. Quaglioni and P. Navrátil, Phys. Lett. B652, 370 (2007), arXiv:0704.1336[nucl-th].

[25] K. Michaelian et al., Phys. Rev. D 41, 2689 (1990).

[26] B. v. Przewoski et al., Phys. Rev. C 45, 2001 (1992).

[27] M. Jetter and H. W. Fearing, Phys. Rev. C 51, 1666 (1995), arXiv:nucl-th/9410040.

[28] J. A. Eden and M. F. Gari, Phys. Rev. C 53, 1102 (1996), arXiv:nucl-th/9506001.

[29] G. H. Martinus, O. Scholten, and J. A. Tjon, Phys. Rev. C 58, 686 (1998).

[30] K. Nakayama and H. Haberzettl, Phys. Rev. C 80, 051001 (2009), arXiv:0910.0469[nucl-th].

[31] K. Yasuda et al., Phys. Rev. Lett. 82, 4775 (1999).

[32] J. G. Messchendorp et al., Phys. Rev. Lett. 82, 2649 (1999).

[33] J. G. Messchendorp et al., Phys. Rev. Lett. 83, 2530 (1999).

[34] H. Huisman et al., Phys. Rev. Lett. 83, 4017 (1999).

[35] H. Huisman et al., Phys. Lett. B476, 9 (2000).

[36] M. Hoefman et al., Phys. Rev. Lett. 85, 1404 (2000).

[37] J. G. Messchendorp et al., Phys. Lett. B481, 171 (2000).

[38] J. G. Messchendorp et al., Phys. Rev. C 61, 064007 (2000).

[39] H. Huisman and N. Kalantar-Nayestanaki, Phys. Rev. C 64, 029801 (2001).

[40] M. Volkerts et al., Phys. Rev. Lett. 90, 062301 (2003).

[41] M. Volkerts et al., Phys. Rev. Lett. 92, 202301 (2004).

[42] M. Mahjour-Shafiei et al., Eur. J. Phys. A 41, 25 (2009), 10.1140/epja/i2009-10786-5.

[43] M. Mahjour-Shafiei et al., Phys. Rev. C 70, 024004 (2004).

[44] A. Johansson and C. Wilkin, Phys. Lett. B673, 5 (2009), arXiv:0812.4250[nucl-ex].

[45] A. A. Mehmandoost-Khajeh-Dad et al., Phys. Lett. B617, 18 (2005), arXiv:nucl-ex/0501012.

[46] J. G. Messchendorp, Mod. Phys. Lett. A24, 810 (2009), arXiv:0810.3939[nucl-ex].

[47] H. Yukawa, Proc. Phys. Math. Soc. Jap. 17, 48 (1935).

[48] W. N. Cottingham, M. Lacombe, B. Loiseau, J. M. Richard, and R. Vinh Mau, Phys. Rev. D 8, 800 (1973).

[49] A. D. Jackson, D. O. Riska, and B. Verwest, Nucl. Phys. A249, 397 (1975).

[50] E. M. Henley and G. A. Miller, Meson Theory of Charge Dependent Nuclear Forces, in Mesons In Nuclei, edited by M. Rho and D. Wilkinson Vol. 1, pp. 405-434, Amsterdam, 1979.

[51] G. A. Miller, A. K. Opper, and E. J. Stephenson, Ann. Rev. Nucl. Part. Sci. 56, 253 (2006), arXiv:nucl-ex/0602021.

[52] R. Machleidt, Phys. Rev. C 63, 024001 (2001), arXiv:nucl-th/0006014.

[53] R. B. Wiringa, V. G. J. Stoks, and R. Schiavilla, Phys. Rev. C 51, 38 (1995), arXiv:nuclth/9408016.

[54] V. G. J. Stoks, R. A. M. Klomp, C. P. F. Terheggen, and J. J. de Swart, Phys. Rev. C 49, 2950 (1994), arXiv:nucl-th/9406039.

[55] G. J. M. Austen and J. J. de Swart, Phys. Rev. Lett. 50, 2039 (1983).

[56] E. A. Uehling, Phys. Rev. 48, 55 (1935).

[57] L. Durand, Phys. Rev. 108, 1597 (1957).

[58] V. G. J. Stoks and J. J. De Swart, Phys. Rev. C 42, 1235 (1990).

[59] R. Machleidt and I. Slaus, J. Phys. G 27, R69 (2001), arXiv:nucl-th/0101056.

[60] F. Gross and A. Stadler, Phys. Rev. C 78, 014005 (2008), arXiv:0802.1552[nucl-th].

[61] J. Haidenbauer, K. Holinde, and M. B. Johnson, Phys. Rev. C 48, 2190 (1993).

[62] A. Deltuva, R. Machleidt, and P. U. Sauer, Phys. Rev. C 68, 024005 (2003).

[63] G. Ecker, Acta Phys. Polon. B38, 2753 (2007), arXiv:hep-ph/0702263.

[64] S. Weinberg, Physica A96, 327 (1979). 
[65] J. Gasser and H. Leutwyler, Ann. Phys. 158, 142 (1984).

[66] V. Bernard, Prog. Part. Nucl. Phys. 60, 82 (2008), arXiv:0706.0312[hep-ph].

[67] S. Weinberg, Phys. Lett. B251, 288 (1990).

[68] S. Weinberg, Nucl. Phys. B363, 3 (1991).

[69] E. Braaten and H. W. Hammer, Phys. Rept. 428, 259 (2006), arXiv:cond-mat/0410417.

[70] E. E. Jenkins and A. V. Manohar, Phys. Lett. B255, 558 (1991).

[71] V. Bernard, N. Kaiser, J. Kambor, and U.-G. Meißner, Nucl. Phys. B388, 315 (1992).

[72] C. Ordóñez, L. Ray, and U. van Kolck, Phys. Rev. C 53, 2086 (1996), arXiv:hep-ph/9511380.

[73] J. L. Friar and S. A. Coon, Phys. Rev. C 49, 1272 (1994).

[74] N. Kaiser, R. Brockmann, and W. Weise, Nucl. Phys. A625, 758 (1997), arXiv:nucl-th/9706045.

[75] E. Epelbaoum, W. Glöckle, and U.-G. Meißner, Nucl. Phys. A637, 107 (1998), arXiv:nuclth/9801064.

[76] N. Kaiser, Phys. Rev. C 64, 057001 (2001), arXiv:nucl-th/0107064.

[77] N. Kaiser, Phys. Rev. C 65, 017001 (2002), arXiv:nucl-th/0109071.

[78] N. Kaiser, Phys. Rev. C 61, 014003 (2000), arXiv:nucl-th/9910044.

[79] N. Kaiser, Phys. Rev. C 62, 024001 (2000), arXiv:nucl-th/9912054.

[80] D. R. Entem and R. Machleidt, Phys. Rev. C 66, 014002 (2002), arXiv:nucl-th/0202039.

[81] T. Becher and H. Leutwyler, Eur. Phys. J. C 9, 643 (1999), arXiv:hep-ph/9901384.

[82] R. Higa and M. R. Robilotta, Phys. Rev. C 68, 024004 (2003), arXiv:nucl-th/0304025.

[83] R. Higa, M. R. Robilotta, and C. A. da Rocha, Phys. Rev. C 69, 034009 (2004), arXiv:nuclth/0310011.

[84] N. Fettes, U.-G. Meißner, and S. Steininger, Nucl. Phys. A640, 199 (1998), arXiv:hep$\mathrm{ph} / 9803266$.

[85] N. Fettes, V. Bernard, and U.-G. Meißner, Nucl. Phys. A669, 269 (2000), arXiv:hep-ph/9907276.

[86] P. Büttiker and U.-G. Meißner, Nucl. Phys. A668, 97 (2000), arXiv:hep-ph/9908247.

[87] D. R. Entem and R. Machleidt, Phys. Rev. C 68, 041001 (2003), arXiv:nucl-th/0304018.

[88] E. Epelbaum, W. Glöckle, and U.-G. Meißner, Nucl. Phys. A747, 362 (2005), arXiv:nuclth/0405048.

[89] V. G. J. Stoks, R. A. M. Kompl, M. C. M. Rentmeester, and J. J. de Swart, Phys. Rev. C 48, 792 (1993).

[90] R. A. Arndt, W. J. Briscoe, I. I. Strakovsky, and R. L. Workman, Phys. Rev. C 76, 025209 (2007), arXiv:0706.2195[nucl-th].

[91] D. B. Kaplan, M. J. Savage, and M. B. Wise, Phys. Lett. B424, 390 (1998), arXiv:nuclth/9801034.

[92] A. Nogga, R. G. E. Timmermans, and U. van Kolck, Phys. Rev. C 72, 054006 (2005), arXiv:nuclth/0506005.

[93] G. P. Lepage, (1997), arXiv:nucl-th/9706029.

[94] J. Gegelia and S. Scherer, Int. J. Mod. Phys. A21, 1079 (2006), arXiv:nucl-th/0403052.

[95] M. C. Birse, Phys. Rev. C 74, 014003 (2006), arXiv:nucl-th/0507077.

[96] M. Pavón Valderrama and E. Ruiz Arriola, Phys. Rev. C 74, 054001 (2006), arXiv:nuclth/0506047.

[97] E. Epelbaum and U.-G. Meißner, (2006), arXiv:nucl-th/0609037.

[98] E. Epelbaum and J. Gegelia, Eur. Phys. J. A 41, 341 (2009), arXiv:0906.3822[nucl-th].

[99] U. van Kolck, J. L. Friar, and J. T. Goldman, Phys. Lett. B371, 169 (1996), arXiv:nuclth/9601009.

[100] J. L. Friar, U. van Kolck, M. C. M. Rentmeester, and R. G. E. Timmermans, Phys. Rev. C 70, 044001 (2004), arXiv:nucl-th/0406026.

[101] E. Epelbaum and U.-G. Meißner, Phys. Rev. C 72, 044001 (2005), arXiv:nucl-th/0502052.

[102] E. Epelbaum, U.-G. Meißner, and J. E. Palomar, Phys. Rev. C 71, 024001 (2005), arXiv:nuclth/0407037.

[103] U. L. Van Kolck, Soft physics: Applications of effective chiral lagrangians to nuclear physics and 
Signatures of three-nucleon interactions in few-nucleon systems

quark models, PhD thesis, University of Texas at Austin, 1993, UMI-94-01021.

[104] T. R. Hemmert, B. R. Holstein, and J. Kambor, J. Phys. G 24, 1831 (1998), arXiv:hep$\mathrm{ph} / 9712496$.

[105] N. Kaiser, S. Gerstendorfer, and W. Weise, Nucl. Phys. A637, 395 (1998), arXiv:nuclth/9802071.

[106] H. Krebs, E. Epelbaum, and U.-G. Meißner, Eur. Phys. J. A32, 127 (2007), arXiv:nuclth/0703087.

[107] E. Epelbaum, H. Krebs, and U.-G. Meißner, Nucl. Phys. A806, 65 (2008), arXiv:0712.1969[nuclth].

[108] J. Klug et al., Nucl. Instr. Meth. Phys. Res. A 489, 282 (2002).

[109] V. Blideanu et al., Phys. Rev. C 70, 014607 (2004), arXiv:nucl-ex/0404039.

[110] J. Rahm et al., Phys. Rev. C 63, 044001 (2001).

[111] C. Brogli-Gysin et al., Nucl. Phys. A541, 137 (1992).

[112] W. Tornow, H. Witała, and A. Kievsky, Phys. Rev. C 57, 555 (1998).

[113] R. T. Braun et al., Phys. Lett. B660, 161 (2008), arXiv:0801.4600[nucl-ex].

[114] F. Gross and A. Stadler, Phys. Lett. B668, 163 (2008), arXiv:0808.2962[nucl-th].

[115] M. R. Robilotta, AIP Conf. Proc. 1011, 1 (2008), arXiv:0803.0521[nucl-th].

[116] H. Primakoff and T. Holstein, Phys. Rev. 55, 1218 (1939).

[117] A. Klein, Phys. Rev. 90, 1101 (1953).

[118] J. Fujita and H. Miyazawa, Prog. Theor. Phys. 17, 360 (1957).

[119] G. Brown and A. Green, Nucl. Phys. A137, 1 (1969).

[120] B. H. McKellar and R. Rajaraman, Phys. Rev. Lett. 21, 450 (1968).

[121] S. A. Coon, M. D. Scadron, and B. R. Barrett, Nucl. Phys. A242, 467 (1975).

[122] S. A. Coon et al., Nucl. Phys. A317, 242 (1979).

[123] J. Carlson, V. R. Pandharipande, and R. B. Wiringa, Nucl. Phys. A401, 59 (1983).

[124] B. S. Pudliner, V. R. Pandharipande, J. Carlson, and R. B. Wiringa, Phys. Rev. Lett. 74, 4396 (1995).

[125] H. T. Coelho, T. K. Das, and M. R. Robilotta, Phys. Rev. C 28, 1812 (1983).

[126] M. R. Robilotta and H. T. Coelho, Nucl. Phys. A460, 645 (1986).

[127] J. L. Friar, D. Huber, and U. van Kolck, Phys. Rev. C 59, 53 (1999), arXiv:nucl-th/9809065.

[128] S. A. Coon and H. K. Han, Few Body Syst. 30, 131 (2001), arXiv:nucl-th/0101003.

[129] A. Nogga, D. Hüber, H. Kamada, and W. Glöckle, Phys. Lett. B409, 19 (1997), arXiv:nuclth/9704001.

[130] R. G. Ellis, S. A. Coon, and B. H. J. McKellar, Nucl. Phys. A438, 631 (1985).

[131] S. A. Coon, M. T. Pena, and D. O. Riska, Phys. Rev. C 52, 2925 (1995), arXiv:nucl-th/9503013.

[132] S. A. Coon, M. T. Pena, and R. G. Ellis, Phys. Rev. C 30, 1366 (1984).

[133] A. Stadler, J. Adam, J., H. Henning, and P. U. Sauer, Phys. Rev. C 51, 2896 (1995), arXiv:nuclth/9504012.

[134] J. Adam, Jr., M. T. Pena, and A. Stadler, Phys. Rev. C 69, 034008 (2004), arXiv:nuclth/0308032.

[135] R. Skibiński et al., (2011), arXiv:1101.2150[nucl-th], accepted for publication in Eur. Phys. J. A.

[136] A. Deltuva, K. Chmielewski, and P. U. Sauer, Phys. Rev. C 67, 034001 (2003).

[137] A. Deltuva, K. Chmielewski, and P. U. Sauer, Phys. Rev. C 67, 054004 (2003).

[138] A. Deltuva, A. C. Fonseca, and P. U. Sauer, Phys. Rev. C 72, 054004 (2005), arXiv:nuclth/0509034.

[139] A. Deltuva, A. C. Fonseca, and P. U. Sauer, Phys. Rev. C 71, 054005 (2005), arXiv:nuclth/0503012.

[140] A. Kievsky, M. Viviani, and S. Rosati, Phys. Rev. C 64, 024002 (2001), arXiv:nucl-th/0103058.

[141] A. Deltuva et al., Phys. Rev. C 71, 064003 (2005), arXiv:nucl-th/0503015.

[142] A. Deltuva, Phys. Rev. C 80, 064002 (2009), arXiv:0912.0240[nucl-th]. 
[143] H. Witała, R. Skibiński, J. Golak, and W. Glöckle, Eur. Phys. J. A 41, 369 (2009), arXiv:0903.1522[nucl-th].

[144] H. Witała, R. Skibiński, J. Golak, and W. Glöckle, Eur. Phys. J. A 41, 385 (2009).

[145] H. W. Fearing and S. Scherer, Phys. Rev. C 62, 034003 (2000), arXiv:nucl-th/9909076.

[146] W. N. Polyzou and W. Glöckle, Few Body Syst. 9, 97 (1990).

[147] A. Amghar and B. Desplanques, Nucl. Phys. A585, 657 (1995).

[148] A. Stadler and F. Gross, Phys. Rev. Lett. 78, 26 (1997), arXiv:nucl-th/9607012.

[149] J. A. Eden and M. F. Gari, Phys. Rev. C 53, 1510 (1996), arXiv:nucl-th/9601025.

[150] V. Efimov and E. G. Tkachenko, Phys. Lett. B157, 108 (1985).

[151] L. Platter, H. W. Hammer, and U.-G. Meißner, Phys. Lett. B607, 254 (2005), arXiv:nuclth/0409040.

[152] H. Witała et al., Phys. Rev. C 63, 024007 (2001), nucl-th/0010013.

[153] P. Navrátil and W. E. Ormand, Phys. Rev. C 68, 034305 (2003), arXiv:nucl-th/0305090.

[154] S. C. Pieper, V. R. Pandharipande, R. B. Wiringa, and J. Carlson, Phys. Rev. C 64, 014001 (2001), arXiv:nucl-th/0102004.

[155] S. Gandolfi, J. Carlson, and S. C. Pieper, Phys. Rev. Lett. 106, 012501 (2011), arXiv:1010.4583[nucl-th].

[156] H. Kamada et al., EPJ Web of Conferences 3, 05025 (2010).

[157] H. Kamada, W. Glöckle, J. Golak, and C. Elster, Phys. Rev. C 66, 044010 (2002), arXiv:nuclth/0208029.

[158] H. Witała, J. Golak, W. Glöckle, and H. Kamada, Phys. Rev. C 71, 054001 (2005), arXiv:nuclth/0412063.

[159] H. Witała, J. Golak, and R. Skibiński, Phys. Lett. B634, 374 (2006), arXiv:nucl-th/0509098.

[160] G. A. Miller and A. Schwenk, Phys. Rev. C 76, 024001 (2007), arXiv:nucl-th/0703018.

[161] H. Witała et al., Phys. Rev. C 77, 034004 (2008), arXiv:0801.0367[nucl-th].

[162] U. van Kolck, Phys. Rev. C 49, 2932 (1994).

[163] E. Epelbaum et al., Phys. Rev. C 66, 064001 (2002), arXiv:nucl-th/0208023.

[164] C. Hanhart, U. van Kolck, and G. A. Miller, Phys. Rev. Lett. 85, 2905 (2000), arXiv:nuclth/0004033.

[165] A. Gårdestig and D. R. Phillips, Phys. Rev. Lett. 96, 232301 (2006), arXiv:nucl-th/0603045.

[166] D. Gazit, S. Quaglioni, and P. Navrátil, Phys. Rev. Lett. 103, 102502 (2009), arXiv:0812.4444[nucl-th].

[167] A. Kievsky, M. Viviani, L. Girlanda, and L. E. Marcucci, Phys. Rev. C 81, 044003 (2010), arXiv:1002.1601[nucl-th].

[168] S. Ishikawa and M. R. Robilotta, Phys. Rev. C 76, 014006 (2007), arXiv:0704.0711[nucl-th].

[169] V. Bernard, E. Epelbaum, H. Krebs, and U.-G. Meißner, Phys. Rev. C 77, 064004 (2008), arXiv:0712.1967[nucl-th].

[170] V. Bernard, E. Epelbaum, H. Krebs, and U.-G. Meißner, in preparation.

[171] L. Girlanda, A. Kievsky, and M. Viviani, (2011), arXiv:1102.4799[nucl-th].

[172] T. Clegg, G. Plattner, L. Keller, and W. Haeberli, Nucl. Instrum. Meth. 57, 167 (1967).

[173] V. Bechtold et al., Nucl. Instrum. Meth. 150, 407 (1978).

[174] T. Wise, A. Roberts, and W. Haeberli, Nucl. Instr. Meth. Phys. Res. A 336, 410 (1993).

[175] T. B. Clegg et al., Nucl. Instr. Meth. Phys. Res. A 357, 212 (1995).

[176] L. Friedrich, E. Huttel, R. Kremers, and A. Drentje, The polarized ion source polis for the agor facility, in Proceedings of the International Workshop on "Polarized beams and polarized gas targets", edited by L. Sydow and H. Paetz gen. Schieck, p. 198, Singapore, 1996, World Scientific.

[177] K. Hatanaka, K. Takahisa, H. Tamura, M. Sato, and I. Miura, Nucl. Instr. Meth. Phys. Res. A 384, 575 (1997).

[178] T. B. Clegg, A Review of Polarized H+- and D+- Ion Source Technology (Invited), in Proceedings of the 2001 Particle Accelerator Conference (PAC2001), edited by P. Lucas and S. Webber 
Vol. 1, pp. 54-58, Chicago, 2001, Institute of Electrical and Electronics Engineers.

[179] H. R. Kremers, J. P. M. Beijers, N. Kalantar-Nayestanaki, and T. B. Clegg, Nucl. Instr. Meth. Phys. Res. A 516, 209 (2004).

[180] J. P. M. Beijers, H. R. Kremers, and N. Kalantar-Nayestanaki, Rev. Sci. Instrum. 77, 03 B505 (2006), 11th International Conference on Ion Sources, Caen, France, Sep 12-16, 2005.

[181] R. Engels et al., Rev. Sci. Instrum. 74, 4607 (2003).

[182] H.-O. Meyer, Ann. Rev. Nucl. Part. Sci. 57, 1 (2007).

[183] R. Bieber et al., Nucl. Instr. Meth. Phys. Res. A 457, 12 (2001).

[184] S. Wells et al., Nucl. Instr. Meth. Phys. Res. A 325, 205 (1993).

[185] L. Sydow et al., Nucl. Instr. Meth. Phys. Res. A 327, 441 (1993).

[186] W. Tornow, R. T. Braun, H. Witala, and N. Koori, Phys. Rev. C 54, 42 (1996).

[187] W. Tornow et al., Nucl. Phys. A631, 421c (1998).

[188] H. R. Setze et al., Phys. Rev. C 71, 034006 (2005).

[189] W. von Witsch and J. Willaschek, Nuclear Instruments and Methods 138, 13 (1976).

[190] T. Köble et al., Nucl. Instr. Meth. Phys. Res. A 275, 460 (1989).

[191] M. Hohlweck et al., Nucl. Instr. Meth. Phys. Res. A 281, 277 (1989).

[192] M. Schöberl, H. Kuiper, R. Schmelzer, G. Mertens, and W. Tornow, Nucl. Phys. A489, 284 (1988).

[193] P. Haffter et al., Nucl. Phys. A548, 29 (1992).

[194] M. T. Gericke et al., (2005), arXiv:nucl-ex/0507005.

[195] Y. Maeda et al., Phys. Rev. C 76, 014004 (2007).

[196] D. González Trotter et al., Nucl. Instr. Meth. Phys. Res. A 599, 234 (2009).

[197] N. Kalantar-Nayestanaki, J. Mulder, and J. Zijlstra, Nucl. Instr. Meth. Phys. Res. A 417, 215 (1998).

[198] S. Abdel-Samad, K. Kilian, J. Ritman, and M. Abdel-Bary, Nucl. Instrum. Meth. A556, 20 (2006).

[199] F. Sperisen, A. Berdoz, H.-O. Meyer, R. Barbieri, and R. Bonham, Nucl. Instr. Meth. Phys. Res. A 274, 604 (1989).

[200] A. Berdoz et al., Nucl. Instrum. Methods Phys. Res. B 40-41, 455 (1989).

[201] P. Hempen et al., Phys. Rev. C 57, 484 (1998).

[202] H. J. Wörtche, Nucl. Phys. A687, 321 (2001), arXiv:nucl-ex/0007015.

[203] H. Amir-Ahmadi et al., Nucl. Instr. Meth. Phys. Res. A 562, 338 (2006).

[204] S. Noji et al., Nucl. Instr. Meth. Phys. Res. A 578, 267 (2007).

[205] H. Sakai et al., Nucl. Instr. Meth. Phys. Res. A 369, 120 (1996).

[206] J. Cameron et al., Nucl. Instr. Meth. Phys. Res. A 305, 257 (1991).

[207] T. Rinckel et al., Nucl. Instrum. Meth. A439, 117 (2000).

[208] E. Steffens and W. Haeberli, Rep. Prog. Phys. 66, 1887 (2003).

[209] F. Rathmann, Nucl. Instr. Meth. Phys. Res. A 536, 235 (2005), Polarized Sources and Targets for the 21st Century. Proceedings of the 10th International Workshop on Polarized Sources and Targets.

[210] W. Meyer, Nucl. Instr. Meth. Phys. Res. A 526, 12 (2004), Proceedings of the ninth International Workshop on Polarized Solid Targets and Techniques.

[211] H. Paetz gen. Schieck, H. Witała, J. Golak, J. Kuroś, and R. Skibiński, Few Body Syst. 30, 81 (2001).

[212] V. Huhn et al., Phys. Rev. C 63, 014003 (2001).

[213] M. Allet et al., Phys. Rev. C 50, 602 (1994).

[214] M. Allet et al., Phys. Lett. B376, 255 (1996).

[215] J. Zejma et al., Phys. Rev. C 55, 42 (1997).

[216] K. Bodek et al., Few Body Syst. 30, 65 (2001), 10.1007/s006010170018.

[217] N. Kalantar-Nayestanaki et al., Nucl. Instr. Meth. Phys. Res. A 444, 591 (2000).

[218] S. Kistryn et al., Phys. Rev. C 68, 054004 (2003). 
[219] T. Rinckel et al., Nucl. Instr. Meth. Phys. Res. A 439, 117 (2000).

[220] J. J. Kuroś-Żołnierczuk et al., Few Body Syst. 34, 259 (2004), arXiv:nucl-th/0402030.

[221] R. Großmann et al., Nucl. Phys. A603, 161 (1996).

[222] G. Rauprich et al., Nucl. Phys. A535, 313 (1991).

[223] M. Karus et al., Phys. Rev. C 31, 1112 (1985).

[224] H. Patberg et al., Phys. Rev. C 53, 1497 (1996).

[225] M. Przyborowski et al., Phys. Rev. C 60, 064004 (1999).

[226] C. Düweke et al., Phys. Rev. C 71, 054003 (2005), arXiv:nucl-ex/0412024.

[227] L. Sydow et al., Few Body Syst. 25, 133 (1998), 10.1007/s006010050099.

[228] L. Sydow et al., Nucl. Phys. A567, 55 (1994).

[229] H. Klein et al., Nucl. Phys. A199, 169 (1973).

[230] D. C. Kocher and T. B. Clegg, Nucl. Phys. A132, 455 (1969).

[231] R. Bieber et al., Phys. Rev. Lett. 84, 606 (2000).

[232] K. Ermisch et al., Phys. Rev. Lett. 86, 5862 (2001).

[233] K. Ermisch et al., Phys. Rev. C 68, 051001 (2003), arXiv:nucl-ex/0308012.

[234] K. Ermisch et al., Phys. Rev. C 71, 064004 (2005).

[235] S. Kistryn et al., Phys. Lett. B641, 23 (2006), arXiv:nucl-ex/0607002.

[236] H. R. Amir-Ahmadi et al., Phys. Rev. C 75, 041001 (2007).

[237] E. Stephan et al., Phys. Rev. C 76, 057001 (2007).

[238] H. Mardanpour et al., Eur. Phys. J. A 31, 383 (2007).

[239] A. Ramazani-Moghaddam-Arani et al., Phys. Rev. C 78, 014006 (2008), arXiv:0804.3147[nuclex].

[240] E. Stephan et al., Eur. Phys. J. A 42, 13 (2009).

[241] M. Eslami-Kalantari et al., Mod. Phys. Lett. A 24, 839 (2009).

[242] J. G. Messchendorp, Mod. Phys. Lett. A 24, 810 (2009).

[243] H. Mardanpour et al., Phys. Lett. B687, 149 (2010).

[244] E. Stephan et al., Phys. Rev. C 82, 014003 (2010).

[245] H. Sakai et al., Phys. Rev. Lett. 84, 5288 (2000).

[246] K. Sekiguchi et al., Phys. Rev. C 65, 034003 (2002).

[247] K. Sekiguchi et al., Phys. Rev. C 70, 014001 (2004), arXiv:nucl-ex/0404026.

[248] K. Sekiguchi et al., Phys. Rev. Lett. 95, 162301 (2005), arXiv:nucl-ex/0510005.

[249] S. Kistryn et al., Nucl. Phys. A548, 49 (1992).

[250] J. Balewski et al., Nucl. Phys. A581, 131 (1995).

[251] H. Postma and R. Wilson, Phys. Rev. 121, 1229 (1961).

[252] E. J. Stephenson, H. Witała, W. Glöckle, H. Kamada, and A. Nogga, Phys. Rev. C 60, 061001 (1999).

[253] G. Igo et al., Nucl. Phys. A195, 33 (1972).

[254] K. Kuroda, A. Michalowicz, and M. Poulet, Nucl. Phys. 88, 33 (1966).

[255] R. E. Adelberger and C. N. Brown, Phys. Rev. D 5, 2139 (1972).

[256] M. Garçon et al., Nucl. Phys. A458, 287 (1986).

[257] B. von Przewoski et al., Phys. Rev. C 74, 064003 (2006), arXiv:nucl-ex/0411019.

[258] H. O. Meyer et al., Phys. Rev. Lett. 93, 112502 (2004).

[259] P. Mermod et al., Phys. Rev. C 72, 061002 (2005).

[260] M. B. Chtangeev, Neutron-deuteron elastic scattering and the three-nucleon force, Master's thesis, Massachusetts Institute of Technology, 2005.

[261] W. P. Abfalterer et al., Phys. Rev. Lett. 81, 57 (1998).

[262] H. Witała et al., Phys. Rev. C 59, 3035 (1999), arXiv:nucl-th/9901047.

[263] H. Witała et al., Few Body Syst. 49, 61 (2010).

[264] K. Sekiguchi et al., Phys. Rev. C 79, 054008 (2009).

[265] H. Mardanpour, Investigation of nuclear forces in $d+p$ elastic and $p+d$ break-up reactions at intermediate energies, PhD thesis, University of Groningen, 2008. 
[266] H. Witała, W. Glöckle, D. Hüber, J. Golak, and H. Kamada, Phys. Rev. Lett. 81, 1183 (1998), arXiv:nucl-th/9801018.

[267] S. Nemoto, K. Chmielewski, S. Oryu, and P. U. Sauer, Phys. Rev. C 58, 2599 (1998).

[268] S. Kistryn et al., Phys. Rev. C 72, 044006 (2005), arXiv:nucl-ex/0508012.

[269] E. Stephan et al., Int. J. Mod. Phys. A 26, 725 (2011).

[270] D. R. Entem, R. Machleidt, and H. Witała, Phys. Rev. C 65, 064005 (2002), arXiv:nuclth/0111033.

[271] C. R. Howell et al., Few Body Syst. 2, 19 (1987), 10.1007/BF01078989.

[272] G. Rauprich et al., Few Body Syst. 5, 67 (1988), 10.1007/BF01351270.

[273] F. Sperisen et al., Nucl. Phys. A422, 81 (1984).

[274] H. Witała et al., Few Body Syst. 15, 67 (1993), 10.1007/BF01076348.

[275] H. O. Meyer, Study of polarization observables in pd elastic scattering and dp break-up at intermediate energies, talk at the workshop on "Three-Nucleon Interactions from Few- to Many-Body Systems", TRIUMF, Vancouver, Canada, 2007.

[276] B. von Przewoski et al., Phys. Rev. C 58, 1897 (1998).

[277] J. Kuroś-Żołnierczuk et al., Phys. Rev. C 66, 024004 (2002), arXiv:nucl-th/0203020.

[278] P. Thörngren Engblom et al., AIP Conf. Proc. 768, 65 (2005), arXiv:nucl-ex/0410006.

[279] P. Thörngren Engblom et al., Measurement of spin observables in the pd breakup reaction, Letter-of-Intent No. 202, Cosy PAC \# 37, 2009.

[280] H. McManus and D. O. Riska, Phys. Lett. B92, 29 (1980).

[281] M. R. Robilotta, Phys. Rev. C 31, 974 (1985).

[282] J. A. Tjon, Phys. Lett. B56, 217 (1975).

[283] A. Nogga, H. Kamada, W. Glöckle, and B. R. Barrett, Phys. Rev. C 65, 054003 (2002), arXiv:nucl-th/0112026.

[284] P. Navrátil, V. G. Gueorguiev, J. P. Vary, W. E. Ormand, and A. Nogga, Phys. Rev. Lett. 99, 042501 (2007), arXiv:nucl-th/0701038.

[285] A. Deltuva, A. C. Fonseca, and P. U. Sauer, Phys. Lett. B660, 471 (2008), arXiv:0801.2743[nuclth].

[286] E. Epelbaum, Phys. Lett. B639, 456 (2006), arXiv:nucl-th/0511025.

[287] E. Epelbaum, Eur. Phys. J. A34, 197 (2007), arXiv:0710.4250[nucl-th].

[288] A. Nogga et al., Four-nucleon force contribution to the binding energy of ${ }^{4} \mathrm{He}$, in 19th International IUPAP Conference on Few-Body Problems in Physics, , EPJ Web of Conferences Vol. 3, p. 05006, 2010, Bonn, Germany, August 31 - September 5, 2009.

[289] D. R. Tilley, H. R. Weller, and G. M. Hale, Nucl. Phys. A541, 1 (1992).

[290] H. Kamada et al., Phys. Rev. C 64, 044001 (2001), arXiv:nucl-th/0104057.

[291] P. Navrátil, S. Quaglioni, I. Stetcu, and B. R. Barrett, J. Phys. G 36, 083101 (2009), arXiv:0904.0463[nucl-th].

[292] M. Viviani, A. Kievsky, and S. Rosati, Phys. Rev. C 71, 024006 (2005), arXiv:nucl-th/0408019.

[293] R. Lazauskas, Etude de la diffusion de particules lourdes sur des systèmes atomiques et nucléaires, $\mathrm{PhD}$ thesis, Université Jospeph Fourier, 2003.

[294] R. Lazauskas and J. Carbonell, Phys. Rev. C 70, 044002 (2004), arXiv:nucl-th/0408048.

[295] A. Nogga, P. Navrátil, B. R. Barrett, and J. P. Vary, Phys. Rev. C 73, 064002 (2006), arXiv:nuclth/0511082.

[296] H. Kamada and W. Glöckle, The 4n system: Solutions of yakubovsky equations for bound and scattering states, in Few-body problems in physics, edited by F. Gross, , AIP Conf. Proc. Vol. 334, pp. 848-851, Woodbury, 1995, AIP, XIVth International Conference on Few Body Problems in Physics, Williamsburg, VA, MAY 26-31, 1994.

[297] M. Viviani, S. Rosati, and A. Kievsky, Phys. Rev. Lett. 81, 1580 (1998), arXiv:nucl-th/9807059.

[298] F. Ciesielski, J. Carbonell, and C. Gignoux, Phys. Lett. B447, 199 (1999), arXiv:nuclth/9903062.

[299] F. Ciesielski and J. Carbonell, Phys. Rev. C 58, 58 (1998), arXiv:nucl-th/9804031. 
[300] A. C. Fonseca, Phys. Rev. Lett. 83, 4021 (1999).

[301] R. Lazauskas et al., Phys. Rev. C 71, 034004 (2005), arXiv:nucl-th/0412089.

[302] A. Deltuva and A. C. Fonseca, Phys. Rev. C 75, 014005 (2007), arXiv:nucl-th/0611029.

[303] M. Viviani, A. Kievsky, L. Girlanda, and L. E. Marcucci, Few Body Syst. 45, 119 (2009), arXiv:0812.3547[nucl-th].

[304] T. W. Phillips, B. L. Berman, and J. D. Seagrave, Phys. Rev. C 22, 384 (1980).

[305] M. Viviani, A. Kievsky, S. Rosati, E. A. George, and L. D. Knutson, Phys. Rev. Lett. 86, 3739 (2001), arXiv:nucl-th/0104002.

[306] R. Lazauskas and J. Carbonell, Few Body Syst. 34, 105 (2004), arXiv:nucl-th/0502021.

[307] A. Deltuva and A. C. Fonseca, Phys. Rev. Lett. 98, 162502 (2007), arXiv:nucl-th/0703065.

[308] B. M. Fisher et al., Phys. Rev. C 74, 034001 (2006), arXiv:nucl-ex/0608024.

[309] M. Viviani, L. Girlanda, A. Kievsky, L. E. Marcucci, and S. Rosati, (2010), arXiv:1004.1306[nuclth].

[310] M. Viviani, private communication.

[311] M. T. Alley and L. D. Knutson, Phys. Rev. C 48, 1890 (1993).

[312] A. Deltuva and A. C. Fonseca, (2010), arXiv:1005.1308[nucl-th].

[313] H. M. Hofmann and G. M. Hale, Phys. Rev. C 77, 044002 (2008), arXiv:nucl-th/0512065.

[314] E. Uzu, H. Kamada, and Y. Koike, Phys. Rev. C 68, 061001 (2003), arXiv:nucl-th/0310001.

[315] C. Elster and W. Glöckle, Phys. Rev. C 55, 1058 (1997), arXiv:nucl-th/9610020.

[316] A. M. Micherdzinska et al., Phys. Rev. C 75, 054001 (2007).

[317] A. Nogga et al., Phys. Lett. B639, 465 (2006), arXiv:nucl-th/0602003.

[318] H. Witała and W. Glöckle, Eur. Phys. J. A37, 87 (2008), arXiv:0806.2757[nucl-th].

[319] A. Ramazani-Moghaddam-Arani et al., Phys. Rev. C 83, 024002 (2011), arXiv:1012.4295[nuclex].

[320] M. Bender, P.-H. Heenen, and P.-G. Reinhard, Rev. Mod. Phys. 75, 121 (2003).

[321] S. C. Pieper, R. B. Wiringa, and J. Carlson, Phys. Rev. C 70, 054325 (2004), arXiv:nuclth/0409012.

[322] K. M. Nollett, S. C. Pieper, R. B. Wiringa, J. Carlson, and G. M. Hale, Phys. Rev. Lett. 99, 022502 (2007), arXiv:nucl-th/0612035.

[323] P. Navrátil and W. E. Ormand, Phys. Rev. Lett. 88, 152502 (2002).

[324] P. Navrátil, J. P. Vary, and B. R. Barrett, Phys. Rev. Lett. 84, 5728 (2000), arXiv:nuclth/0004058.

[325] R. Roth and P. Navrátil, Phys. Rev. Lett. 99, 092501 (2007), arXiv:0705.4069[nucl-th].

[326] D. J. Dean, G. Hagen, M. Hjorth-Jensen, T. Papenbrock, and A. Schwenk, Phys. Rev. Lett. 101, 119201 (2008), arXiv:0709.0449[nucl-th].

[327] R. Roth and P. Navrátil, Phys. Rev. Lett. 101, 119202 (2008), arXiv:0801.1484[nucl-th].

[328] S. Quaglioni and P. Navrátil, Phys. Rev. Lett. 101, 092501 (2008), arXiv:0804.1560[nucl-th].

[329] S. Quaglioni and P. Navrátil, Phys. Rev. C 79, 044606 (2009), arXiv:0901.0950[nucl-th].

[330] P. Navrátil, R. Roth, and S. Quaglioni, Phys. Rev. C 82, 034609 (2010), arXiv:1007.0525[nuclth].

[331] P. Navratil and S. Quaglioni, (2011), arXiv:1102.2042[nucl-th].

[332] E. Epelbaum, H. Krebs, D. Lee, and U.-G. Meißner, Phys. Rev. Lett. 104, 142501 (2010), arXiv:0912.4195[nucl-th].

[333] B. Borasoy, E. Epelbaum, H. Krebs, D. Lee, and U.-G. Meißner, Eur. Phys. J. A34, 185 (2007), arXiv:0708.1780[nucl-th].

[334] E. Epelbaum, H. Krebs, D. Lee, and U.-G. Meißner, Eur. Phys. J. A 45, 335 (2010), arXiv:1003.5697[nucl-th].

[335] B. Borasoy, E. Epelbaum, H. Krebs, D. Lee, and U.-G. Meißner, Eur. Phys. J. A35, 357 (2008), arXiv:0712.2993[nucl-th].

[336] E. Epelbaum, H. Krebs, D. Lee, and U.-G. Meißner, (2011), arXiv:1101.2547v1[nucl-th].

[337] R. Roth et al., Phys. Rev. C 73, 044312 (2006), arXiv:nucl-th/0510036. 
[338] L. Coraggio, A. Covello, A. Gargano, and N. Itaco, (2010), arXiv:1005.2896[nucl-th].

[339] S. K. Bogner, A. Schwenk, R. J. Furnstahl, and A. Nogga, Nucl. Phys. A763, 59 (2005), arXiv:nucl-th/0504043.

[340] L. Tolos, B. Friman, and A. Schwenk, Nucl. Phys. A806, 105 (2008), arXiv:0711.3613[nucl-th].

[341] K. Hebeler, S. K. Bogner, R. J. Furnstahl, A. Nogga, and A. Schwenk, Phys. Rev. C 83, 031301 (2011), arXiv:1012.3381[nucl-th].

[342] S. Bogner, R. Furnstahl, and A. Schwenk, (2009), 0912.3688.

[343] H. Kümmel, K. H. Lührmann, and J. G. Zabolitzky, Phys. Rept. 36, 1 (1978).

[344] B. Mihaila and J. H. Heisenberg, Phys. Rev. C 61, 054309 (2000), arXiv:nucl-th/9912023[nuclth].

[345] S. A. Coon, J. G. Zabolitzky, and D. W. E. Blatt, Z. Phys. A 281, 137 (1977), $10.1007 / \mathrm{BF} 01408625$.

[346] G. Hagen et al., Phys. Rev. C 76, 034302 (2007), arXiv:0704.2854[nucl-th].

[347] S. A. Coon, Int. Phys. Conf. Ser. 86, 523 (1986), Proceedings of the Int. Nucl. Phys. Conf., Harrogate, UK.

[348] G. Hagen, T. Papenbrock, D. J. Dean, and M. Hjorth-Jensen, Phys. Rev. C 82, 034330 (2010), arXiv:1005.2627[nucl-th].

[349] H. Feldmeier and J. Schnack, Rev. Mod. Phys. 72, 655 (2000), arXiv:cond-mat/0001207[condmat].

[350] N. Barnea, W. Leidemann, and G. Orlandini, Nucl. Phys. A693, 565 (2001), arXiv:nuclth/0101050.

[351] M. Chernykh, H. Feldmeier, T. Neff, P. von Neumann-Cosel, and A. Richter, Phys. Rev. Lett. 98, 032501 (2007).

[352] N. Barnea, V. D. Efros, W. Leidemann, and G. Orlandini, Few Body Syst. 35, 155 (2004), arXiv:nucl-th/0404086.

[353] N. Barnea, W. Leidemann, and G. Orlandini, Phys. Rev. C 81, 064001 (2010).

[354] S. Bacca, H. Arenhoevel, N. Barnea, W. Leidemann, and G. Orlandini, Phys. Lett. B603, 159 (2004), arXiv:nucl-th/0406080.

[355] P. Navrátil and B. R. Barrett, Phys. Rev. C 57, 3119 (1998), arXiv:nucl-th/9804014.

[356] B. S. Pudliner, V. R. Pandharipande, J. Carlson, S. C. Pieper, and R. B. Wiringa, Phys. Rev. C 56, 1720 (1997), arXiv:nucl-th/9705009.

[357] S. C. Pieper, K. Varga, and R. B. Wiringa, Phys. Rev. C 66, 044310 (2002), arXiv:nuclth/0206061.

[358] R. B. Wiringa, private communication.

[359] P. Doleschall and I. Borbely, Phys. Rev. C 62, 054004 (2000).

[360] A. Shirokov, J. Vary, A. Mazur, and T. Weber, Phys. Lett. B644, 33 (2007).

[361] P. Navrátil, Nuclear structure from first principles: Ab initio no-core shell model for light nuclei, talk at the "CNS International Summer School (CISS04)", 2004.

[362] S. K. Bogner, T. T. S. Kuo, and A. Schwenk, Phys. Rept. 386, 1 (2003), arXiv:nucl-th/0305035.

[363] E. Epelbaum, W. Glöckle, and U.-G. Meißner, Phys. Lett. B439, 1 (1998), arXiv:nuclth/9804005.

[364] S. Fujii et al., Phys. Rev. C 70, 024003 (2004), arXiv:nucl-th/0404049.

[365] S. K. Bogner, R. J. Furnstahl, and R. J. Perry, Phys. Rev. C 75, 061001 (2007), arXiv:nuclth/0611045.

[366] R. Roth, T. Neff, H. Hergert, and H. Feldmeier, Nucl. Phys. A745, 3 (2004), arXiv:nuclth/0406021.

[367] A. Nogga, S. K. Bogner, and A. Schwenk, Phys. Rev. C 70, 061002 (2004), arXiv:nuclth/0405016.

[368] S. K. Bogner, R. J. Furnstahl, and A. Schwenk, Prog. Part. Nucl. Phys. 65, 94 (2010), arXiv:0912.3688[nucl-th].

[369] A. Gunther, R. Roth, H. Hergert, and S. Reinhardt, (2010), arXiv:1005.1599[nucl-th]. 
[370] S. K. Bogner et al., Nucl. Phys. A801, 21 (2008), arXiv:0708.3754[nucl-th].

[371] E. D. Jurgenson, P. Navratil, and R. J. Furnstahl, Phys. Rev. Lett. 103, 082501 (2009), arXiv:0905.1873[nucl-th].

[372] T. Otsuka, T. Suzuki, J. D. Holt, A. Schwenk, and Y. Akaishi, Phys. Rev. Lett. 105, 032501 (2009), arXiv:0908.2607[nucl-th].

[373] A. C. Hayes, P. Navrátil, and J. P. Vary, Phys. Rev. Lett. 91, 012502 (2003), arXiv:nuclth/0305072.

[374] P. Maris et al., (2011), arXiv:1101.5124[nucl-th].

[375] J. W. Holt, N. Kaiser, and W. Weise, Phys. Rev. C 79, 054331 (2009), arXiv:0901.4750[nucl-th].

[376] J. W. Holt, G. E. Brown, T. T. S. Kuo, J. D. Holt, and R. Machleidt, Phys. Rev. Lett. 100, 062501 (2008), arXiv:0710.0310[nucl-th].

[377] R. Machleidt, (2009), arXiv:0909.2881[nucl-th]. 